ELECTROMAGNETIC SCATTERING BY A TWO-DIMENSIONAL INHOMOGENEITY DUE TO AN OSCHLATING MAGNETIC DIPOLE

\begin{tabular}{|c|c|}
\hline \multirow{2}{*}{$\begin{array}{l}\text { Ki Ha Lee } \\
(P h .0 \text {. Thes Is) }\end{array}$} & 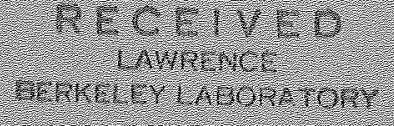 \\
\hline & 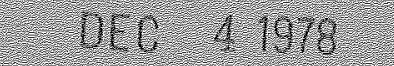 \\
\hline eptember is & DOCUMENTS \\
\hline
\end{tabular}

Prepared for the U. S. Department of Energy under Contract $\mathbf{N}-7405-E N G-M 8$

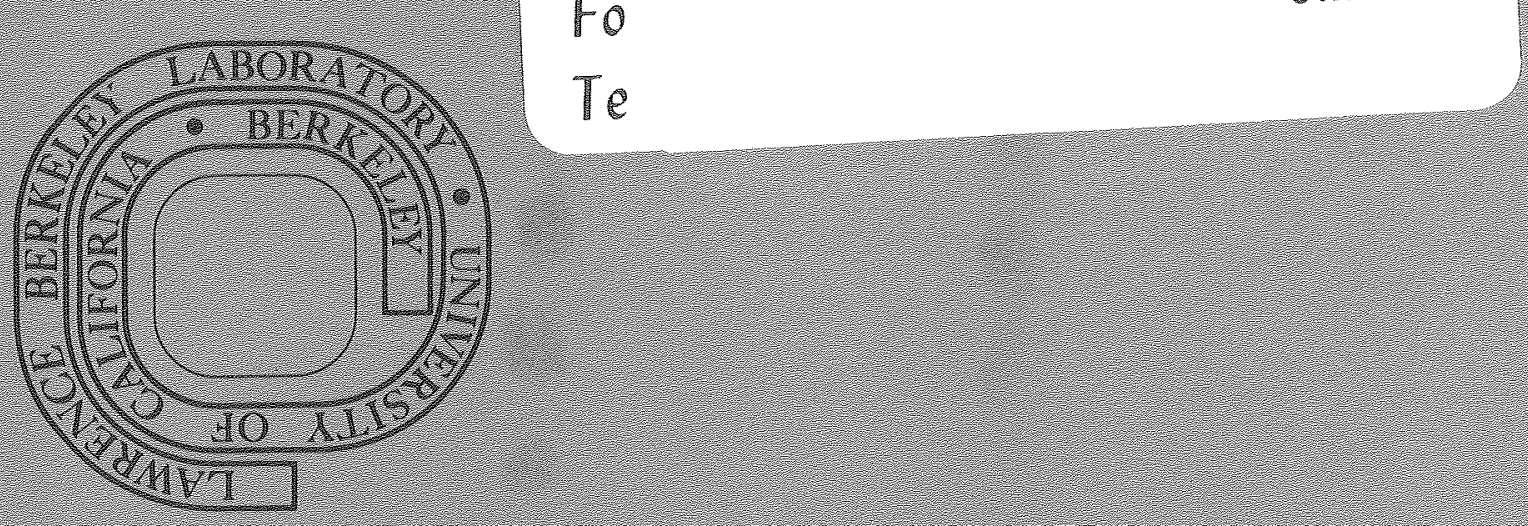

opy o weeks. wh

Fo call 


\section{LEGAL NOTIOE}

This repert was prepared as an accown or work sponsored by tho Whited Shates Government. Neither hlo thiled Stares not he Depant: ment of Etrergy, nor any of their employees, nor any of their contractors, suboontractors or theil emoloyecs. makes any watranty. express or implied or assumes any legal llability or responsilulity for the qcouracy completeness of userulness of any information, apparants protuct of process diselesed or represents that its use would hot ininge orwately owned rights: 


\title{
Electromagnetic Scattering by a Two-Dimensional Irhomogeneity \\ Due to an Oscillating Magnetic Dipole
}

By

Ki Ha Lee

\author{
Engineering Geoscience \\ Materials Science and Engineering \\ University of California \\ Berkeley, California 94720 \\ and \\ Earth Sciences Division \\ Lawrence Berkeley Laboratory \\ Berkeley, California 94720
}

Work performed under the auspices of the U.S. Department of Energy. 



\begin{abstract}
A numerical method of computing the electromagnetic response of two-dimensional earth models to an oscillating magnetic dipole is presented. The generalized electromagnetic variational integral is rem duced to a sum of two-dimensional variational integrals by fourier transformation. Discretization of each two dimensional integral is carried out in terms of the secondary electric fields using the finite element method. Following the variational principle, each harmonic in tegral is reduced to a set of simultaneous equations. From each set of electric field solutions obtained by solving the simultaneous equations, the secondary magnetic fields are computed numerically. After inversely Fourier transforming the secondary electric and magnetic fields, the total fields are finally obtained by adding the analyticalo ly calculated primary fields.

In order to formulate the source vector associated with each set of simultaneous equations, both the secondary electric fields along the external boundary and the primary electric fields at every node must be
\end{abstract}


using an algorithm developed for this purpose. The secondary field used is the total field with the free space dipolar field subtracted. When a horizontal magnetic dipole is located close to or on the surface of the earth, it is found that the solution obtained in the manner described is greatly in error. This is mainly due to the inadequately assumed primary field behaviour within the elements in the immediate vicinity of source. An acceptable solution has been obtained by initially increasing the accuracy of integrations over these elements. Because of the systematically implied continuity of the electric field in the finite element solution, the given discontinuous conductivity is modified to a continuous one across internal boundaries. In the presence of a horizontal magnetic dipole, however, the charge distribution across the air-to-earth interface is so abrupt, even with this modification, that there appears to be a numerical limit beyond which the proposed method can not cope. Consequently, the quality of the solution for the horizontal magnetic dipole is found to be relam tively poor compared to that for the vertical magnetic dipole.

Over a uniform half space test model, the internal consistency of the method developed in this thesis is demonstrated by ensuring: 1) the radial symmetry of the horizontal electric fields in the presence of a vertical magnetic dipole, 2) the reciprocity between field components, $H_{x}$ due to a vertical magnetic dipole and $H_{z}$ due to a horizontal magnetic dipole. The reciprocity check is also made on a two dimensional model. In addition, the numerical solutions for a two-layered half space model in the presence of a vertical magnetic dipole are shown to match very well the analytic solutions obtained for a few significant frequencies. 
It is not possible to perform an absolute numerical check of the solution due to the lack of another independently developed solution against which it can be checked. As an alternative the solutions for two-dimensional models have been compared to those for some elongated three-dimensional models whose cross sections correspond to the twomim mensional models. Three-dimensional model results from a scaled tank model and a numerical solution obtained using the finite element method have been used for this check. Finally, the response of a contact model has been investigated using a transmitter-receiver pair with a fixed separation traversed along the surface of the earth. 
ACKNOWLEDGMENTS

The author would Tike to acknowledge the financial assistance provided by the U.S. Department of Energy through the University of California - Lawrence Berkeley Laboratory.

I sincerely wish to express my gratitude to Professor H.F. Morrison and Dr. D.F. Pridmore for their valuable advice and suggestions leading to the conclusion of this work. 
TABLE OF CONTENTS

\section{INTRODUCTION}

I. Formulation of the Variational Integral

II. Harmonic Version of the Variational Integral

A. Two-Dimensional Variational Integral

B. Derivation of the Wave Equation for the Secondary Field

III. Field Approximation

A. Finite Element Method

B. Boundary Condition

C. Source Vector

IV. Evaluation of the Numerical Solution

A. Secondary Field Solution

B. Numerical Check

V. Conclusion

APPENDIX I. Reduction of Variational Integral to Differential Equation

APPENDIX II. Soft Boundary

APPENDIX III. The Harmonic Electromagnetic Field due to a Magnetic Dipole

REFERENCES 



\section{INTRODUCTION}

The electromagnetic method has been used in a variety of geophy sical situations especially in mining exploration. A review of the common techniques has been presented by ward (196\%). The interpretation of electromagnetic data collected in the field has been based on either the theoretical solutions for simple models in free space (e.g., Grant and West, 1965) or on measurenents made with scaled models (e.g., Guar et a1, 1972: Sarma and Maru, 1971). Only a limited number of model tank results are available due to the difficulty of finding the right combinations of conductive materials that can be used to model realistic geologic structures. Theoretical solutions for layered half spaces have been given by Wait (1962), Frischknecht (1967), Dey and Ward (1970) and Ryu et al (1970), to list only a few. Except for a few simple bodies of nigher order symmetry in shape such as spheres or cylinders, all the electromagnetic scattering problems must be solved numerically.

The two-dimensional electromagnetic scattering problems have been studied by various authors; Patrick and Bostic (1969), Hohmann (1970), Parry and Ward (1971), Ryu (1971), and Coggon (1971). The solutions presented by these authors have been restricted to geophysical exploration techniques in which the source field is either uniform (magnetotellurics) or two-dimensional (e.g., an infinite wire, Turam, source). Recent7y, Weidelt (1975), Hohmann (1975), and Meyer (1977), have found solutions for three-dimensional scattering problems with arbitrary source using integral equations. Pridmore (1978) also has given a finite element solution for the scattering by a three-dimensional inhomogeneity buried in a conductive half space. Unfortunately, except for 
a few models with specific conductivity contrasts, disagreements exist among solutions for the same model with different techniques. The three dimensional integral equation technique can not, for example, be used if the source is sitting right on a surface inhomogeneity.

Geologic models in which the electrical parameters are invarient with strike constitute an important class of targets for electromagnetic exploration. Fault contacts, large tabular bodies (dykes, contact ore zones), etc., are models that have not yet been successfully treated for exploration purposes using dipolar source fields. Although these geological structures can be modelled by one of the three-dimensional modelling techniques using either the finite difference or the finite element method, the computing cost is prohibitive. On the other hand, we have not been able to use the integral equation technique on these models.

In this thesis a solution has been developed for the electromagnetic scattering by an arbitrary two-dimensional distribution of subsurface conductivity due to the excitation of a magnetic dipole located on or above the earth surface. The problem is essentially three-dimensional, but since the half space geometry is two-dimensional in terms of the electric constants, the electromagnetic problem can be reduced to two dimensions by fourier transformation. The theoretical basis of the method is the variational principle. The variational integral, the total electromagnetic energy contained in the prescribed system, is minimized on a discretized grid of finite elements which is used to approximate the continuous distribution of conductivity in the ground. The preliminary solution is obtained in terms of the secondary electric 
field in harmonic space. The final solution is the inversely Fourier transformed secondary field plus the primary field calculated analytical1y.

This technique was proposed by Ryu (1971) in his thesis using a finite element method. A triangle was used as the fundamental element. He formulated the system equation in terms of the magnetic fields, but stopped short of attempting to obtain a solution mainly due to the fact that his intended number of narmonic solutions was too great to be practically undertaken. Stoyer (1974) has developed a finite difference solution for the same geophysical problem with the coupled transmission sheet analogy. Mathematically as well as physically, his algorithm is superior to the one presented in this thesis. All the electromagnetic field components are expressed in terms of two field variables, $H_{y}$ and $E_{y}$, polarized in the direction parallel to the strike. Hence, it seems that there is no discontinuous field directly involved in the system equation. However, his method has the following major drawbacks; 1) since all but $H_{y}$ and $E_{y}$ are expressed in terms of combinations of the derivatives of the two field components, the quality of the discretized numerical solution tends to be degraded, 2) his source vector, basically obtained by taking derivatives of the delta function, would lead to a solution in which a substantial error would appear in the magnitude, but not necessary in the tilt angle or the ellipticity of the magnetic field, and 3) he used terminal impedance for boundary condition assuming that the electromagnetic fields at the boundary are plane waves. This assumption is generally not acceptable, since even in the most optimistic situation the radiation field behaves proportionally to $1 / r$ across the boundary. It is obvious that 
the closer the source approaches to the boundary, the more contaminated the solution would become with this boundary condition.

The first two parts of the thesis have been allocated for the derivation of two-dimensional harmonic variational integral. A rigorous illustration of the relationship between a variational integral and the corresponding integral equation has been given. The algorithm leading to the finite element numerical solution is prescribed in the third part. The formulation of the source vector as well as the selection of proper boundary conditions is also discussed in this part. The numerical solutions are presented in the fourth part, and finally the results are compared to the existing analytic or numer ical solutions. 
I. Formulation of the Variational Integral

The generalized geophysical electromagnetic system is illustrated in Figure 1. The inhomogeneity consists of a distribution of anomalous conductivity $\sigma_{2}$ as a function of position. The electric permittivity $\varepsilon$ and the magnetic permeability $\mu$ have been assumed to be those values found in vacuum. A monochromatic time dependence of $e^{\text {jwt }}$ has been assumed for all the field variables.

Following Morse and Feshbach (1953), the electromagnetic field behaves in such a way that the total electromagnetic energy contained in the system is stationary. Therefore once the energy is expressed in terms of field variables, usually in an integral form, the desired solution can be obtained by finding the stationary point where the variation of the total energy vanishes.

Using Maxwell's equations,

$$
\begin{aligned}
& \nabla \times \bar{E}=-\frac{\partial \bar{B}}{\partial \bar{C}}, \\
& \nabla \times \bar{H}=\bar{J}+\frac{\partial \bar{B}}{\partial \bar{C}}, \\
& \nabla \cdot \bar{B}=0, \\
& \nabla \cdot \bar{D}=\rho,
\end{aligned}
$$

and the constitutive relations and $0 \mathrm{hm}^{\prime} \mathrm{s}$ Iaw,

$$
\bar{B}=\mu \bar{H}, \quad \bar{D}=\varepsilon \bar{E}, \quad \bar{J}=\sigma \bar{E},
$$

Stratton (1941) has shown that

$$
-\int_{S} \bar{E} \times \bar{H} \cdot \bar{n} d a=\int_{v} \sigma \bar{E}^{2} d v+\frac{\partial}{\partial t} \int_{v}\left(\frac{\varepsilon}{2} \bar{E}^{2}+\frac{\mu}{2} \bar{H}^{2}\right) d v \text {. }
$$

This result was first derived by Poynting and Heaviside separately in 1884. Its conventional interpretation is that the right hand side of $(1-6)$, the time rate of increase of Joule heat loss and of electric and 


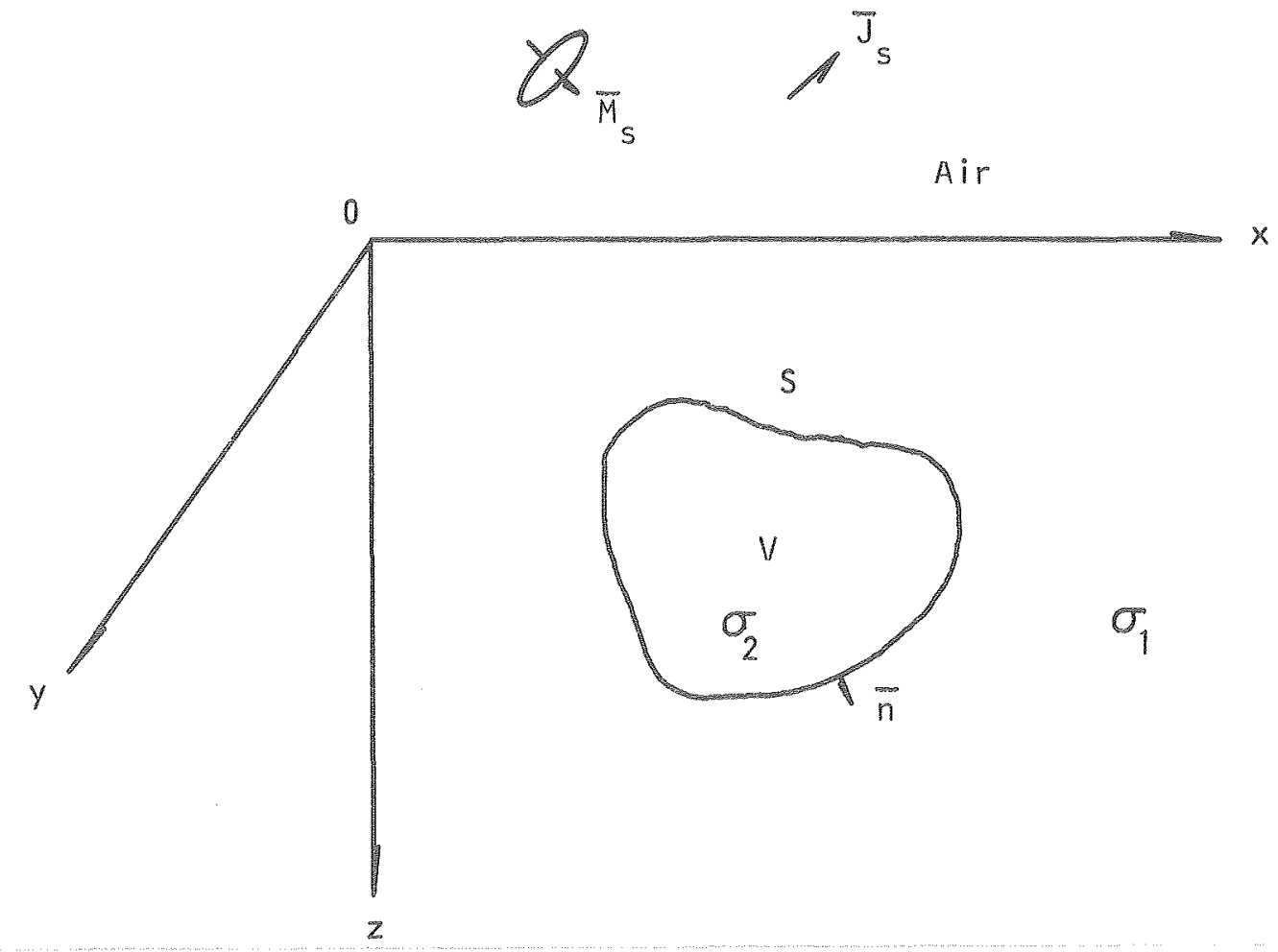

Figure 1. A generalized geophysical electromagnetic system

XBL 789-10945 
magnetic energy stored within the volume $V$, is exactly compensated by the left hand side, the time rate of electromagnetic energy flowing inward across the closed surface, $S$, bounding $V$.

Upon integrating the right hand side of equation $(I-6)$ with respect to time, we obtain the electromagnetic energy contained in the volume in the frequency domain. In an active system where the system is excited by a finite source, the source energy must be included in the variational integral. Finally it can be shown that the variational integral $I(\bar{E}, \bar{H})$, the total electromagnetic energy, is written as

$$
I(\bar{E}, \bar{H})=\int v\left(\frac{\mu}{2} \bar{H}^{2}+\frac{\varepsilon}{2} \bar{E}^{2}+\frac{\sigma}{2 j \omega} \bar{E}^{2}+\mu \bar{H} \cdot \bar{M}_{s}+\frac{1}{j \omega} \bar{E} \cdot \bar{J}_{s}\right) d v \text {, }
$$

where $\bar{M}_{S}$ and $\bar{J}_{s}$ are a magnetic dipole moment density and a source current density, respectively. Equation (I-7) may be further simplified by unifying the integrand variables in $\vec{E}$ or in $\vec{H}$ depending upon the type of solution desired. If the solution is sought in magnetic field, equation (I-7) becomes

$$
I(\bar{H})=\int_{v}\left\{\frac{\mu}{2} \bar{H}^{2}-\frac{\mu}{2 k^{2}}(\nabla \times H)^{2}+\mu \bar{H} \cdot \bar{M}_{s}\right\} d v,
$$

whereas, it can be rewritten in electric field as

$$
I(E)=\int_{v}\left\{\frac{k^{2}}{2 \omega^{2} \mu} \bar{E}^{2}-\frac{1}{2 \omega^{2} \mu}(\nabla \times \bar{E})^{2}+\frac{1}{j \omega} \bar{E} \cdot \bar{J}_{s}\right\} d v,
$$

where $k$ is the propagation constant defined by

$$
k^{2}=\omega^{2} \mu \varepsilon-j \sigma \omega \mu
$$

The two variational integrals $(I-8)$ and $(I-9)$ are physically equivalent in the sence that both represent the same energy contained in the prescribed system.

It can be shown, Appendix I, that the stationary condition imposed upon the variational integrals $I(\bar{H})$ and $I(E)$ results in the following 
differential equations governing $\bar{H}$ and $\bar{E}$, respectively:

$$
\begin{aligned}
& \bar{H}-\frac{1}{k^{2}} \nabla \times \nabla \times \bar{H}=-\bar{M}_{s}, \\
& k^{2} \bar{E}-\nabla \times \nabla \times \bar{E}=j \omega \mu \bar{J}_{s} .
\end{aligned}
$$

From an analytical point of view, either of these equations produces an analytic solution provided that the given geometry guarantees its existence. Since there exists one analytic solution exclusively in each one of the subdomains separated by a number of internal boundaries, there are in general two solutions at the internal boundary as the field point is approached from both directions. Whether the solutions are identical or not is strictly dependent upon the boundary condition deduced from the physical property of the field itself. Electric fields, for example, undergo step discontinuities at the boundary between regions of different conductivity.

Within the context of a discretized numerical scheme however, we are not a 71 owed to have two solutions at a nodal point. Consequently, in the absence of magnetic inhomogeneities, it would appear that the continuity of magnetic fields would make variational integral (I-8) the right choice for our purpose. Unfortunately, we have encountered a severe numerical probiem with this equation in the computing process. As can be noticed from equation (I-11), the magnitude of the coefficient of $\nabla \times \nabla \times \vec{H}$ term decreases proportionally from $1 / \omega \varepsilon$ to $1 / \sigma$ across the air-to - earth interface. For common geological situations, a typical contrast of this coefficient across the interface is somewhere between $10^{5}$ to $10^{9}$. This is believed to have been the major reason why proper couplings among three components of magnetic fields could not, practically, be achieved across the interface in the numerical scheme. The same difficulty has 
been found by Pridmore (1978) in his development of a finite element solution for 3-D electromagnetic scattering problem.

We have thus decided to seek solutions in electric fields using equation (I-9). In order to eliminate the apparent contradiction inherently associated with the electric field solution discussed earlier, we have substituted a narrow zone for the region of discontinuous conductivity across an internal boundary. Within this zone a smooth transition of conductivity change has been imposed. The modification can be easily extended to a cornered boundary. Appendix 11 shows the detailed modification procedure and some of the necessary integrations for variational integrals. 
II. Harmonic Version of Variational Integral

A. Twodimensional variational integral

In part I we have discussed how the generalized variational integral is formulated in 3-D. A particular version of the variational in tegral, the one in terms of electric field, has been selected on the basis of numerical consideration.

Given a two-dimensional geometry, however, we can find the solution by initially Fourier transforming the system and summing up a finite number of solutions obtained in harmonic space. Stoyer (1974) presented a finite difference solution using the fourier transform technique. Ryu (1971), using a finite element nethod, attempted to formulate essentialTy the same problem presented here. Using the same technique, we will first transform out the strike directional dependence.

To begin, we have chosen a magnetic dipole source oriented in the direction perpendicular to the strike. With reference to figure it is assumed that the $y$ axis is the strike direction. The symetry of every electromagnetic field component stays unchanged in $y$ so long as the source is oriented in this direction $\left(i . \mathrm{e}_{2}, \mathrm{H}_{\mathrm{x}}, \mathrm{H}_{2}\right.$, and $\mathrm{E}_{\mathrm{y}}$ are symmetric).

Using the Fourier integral and the appropriate symmetry conditions in $y$, we may write:

$$
\begin{aligned}
& E_{x}(x, y, z)=\frac{j}{\pi} \int_{0}^{\infty} E_{x}\left(x, k_{y}, z\right) \text { sink ky d ky, } \\
& E_{y}(x, y, z)=\frac{1}{\pi} \int_{0}^{\infty} E_{y}\left(x, k_{y}, z\right) \cos k_{y} y d k_{y}, \\
& E_{z}(x, y, z)=\frac{j}{\pi} \int_{0}^{\infty} E_{z}\left(x, k_{y}, z\right) \sin k_{y} y d k_{y},
\end{aligned}
$$

and 


$$
\begin{aligned}
& H_{x}(x, y, z)=\frac{1}{\pi} \int_{0}^{\infty} H_{x}\left(x, k_{y}, z\right) \cos k_{y} y d k_{y}, \\
& H_{y}(x, y, z)=\frac{j}{\pi} \int_{0}^{\infty} H_{y}\left(x, k_{y}, z\right) \sin k_{y} y d k_{y}, \\
& H_{z}(x, y, z)=\frac{1}{\pi} \int_{0}^{\infty} H_{z}\left(x, k_{y}, z\right) \cos k_{y} y d k_{y},
\end{aligned}
$$

where the transform pair is defined by

$$
P\left(x, k_{y}, z\right)=\int_{-\infty}^{\infty} P(x, y, z) e^{-j k_{y} y} d k_{y} .
$$

If the artificial source is a magnetic dipole $M_{S}$, the source current density $\bar{J}_{s}$ present in the integral (I-9) may be found by

$$
\bar{J}_{s}=\nabla \times \bar{M}_{s} \text {, }
$$

Where

$$
\bar{M}_{s}=\bar{M}_{s} \delta\left(x-x^{\prime}\right) \delta(y) \delta\left(z-z^{\prime}\right) \text {. }
$$

Equation (II-8) is not a very well defined function in view of the delta function associated with $\bar{M}_{S}$. Direct incorporation of the source term $\nabla \times M_{S}$ into a discretized numerical scheme would result in a serious technical problem. The difficulty may be removed if the solution is sought in terms of the secondary field.

To simplify expressions let us use $\bar{J}_{S}$ instead of $\nabla \times \bar{M}_{S}$. The symmetry in $y$ for each of the Cartesian components of $J_{5}$ will be controlled by the transmitting $\tilde{M}_{S}$ and should be the same as that for the electric field. Thus,

$$
\begin{aligned}
& J_{x}(x, y, z)=\frac{j}{\pi} \int_{0}^{\infty} J_{x}\left(x, k_{y}, z\right) \text { sinky } y d k_{y}, \\
& J_{y}(x, y, z)=\frac{1}{\pi} \int_{0}^{\infty} J_{y}\left(x, k_{y}, z\right) \cos k_{y} y d k_{y}, \\
& J_{z}(x, y, z)=\frac{j}{\pi} \int_{0}^{\infty} J_{z}\left(x, k_{y}, z\right) \text { sinkyy d ky. }
\end{aligned}
$$


Instead of directly substituting fourier integrals into the variam tional integral (I-9), we first approximate them using fourier series. Figure 2 shows a corresponding two-dimensional geometry for which an approximate numerical solution is sought. Using fourier cosine and sine series expansions, we have

$$
\begin{aligned}
& P(x, y, z)=\frac{1}{L} \sum_{i=0}^{N} P\left(x, \eta_{i}, z\right) \cos \eta_{i} y, \\
& Q(x, y, z)=\frac{j}{L} \sum_{i=0}^{N} Q\left(x, \eta_{i}, z\right) \sin \eta_{i} y,
\end{aligned}
$$

where $n_{i}=\frac{\pi i}{L}$. The distance $L$ should be long enough to justify our assumption that the half space is two-dimensional.

Substituting fourier series representations of $E$ and $J_{s}$ into equation (1.9), we obtain the following harmonic version of the variational integral: after dropping $\left(x, n_{i}, z\right)$,

$$
\begin{aligned}
& I(\bar{E})=\frac{1}{L^{2}} \int_{V} \frac{k^{2}}{2 \omega^{2} \mu}\left\{\sum_{i=0}^{N}\left(j E_{x} \sin \eta_{i} y \bar{i}_{x}+E_{y} \cos \eta_{i} y E_{y}+j E_{z} \sin \eta_{i} y \bar{i}_{z}\right)\right\}^{2} d v \\
& -\frac{1}{L^{2}} \int_{V} \frac{1}{2 \omega^{2} \mu}\left\{\sum _ { i = 0 } ^ { N } \left[\left\{\frac{\partial}{\partial y}\left(j E_{z} \sin \eta_{i} y\right)-\frac{\partial}{\partial z}\left(E_{y} \cos \eta \dot{y}\right)\right\} \Sigma_{x}\right.\right. \\
& +\left\{\frac{\partial}{\partial}\left(j E_{x} \sin \eta_{i} y\right)-\frac{\partial}{\partial x}\left(j E_{3} \sin \eta_{i} y\right)\right\} E_{y} \\
& \left.\left.+\left\{\frac{\partial}{\partial x}\left(E_{y} \cos \eta_{i} y\right)-\frac{\partial}{\partial y}\left(j E_{x} \sin \eta_{i} y\right)\right\} \tilde{i}_{z}\right]\right\}^{2} d v \\
& +\left.\frac{1}{L^{2}}\right|_{v} \frac{1}{j w}\left\{\sum_{i=0}^{N}\left(j E_{x} \sin \eta_{i} y \bar{i}_{x}+E_{y} \cos \eta_{i} y \bar{i}_{y}+j E_{z} \sin \eta_{i} y \bar{i}_{z}\right)\right\} \\
& \text { - }\left\{\sum_{i=0}^{N}\left(j J_{x} \sin \eta i y \bar{i}_{x}+J_{y} \cos \eta_{i} y \bar{i}_{y}+j J_{y} \sin \eta i y \bar{i}_{z}\right)\right\} d v_{0}
\end{aligned}
$$


$O \bar{M}_{s}$

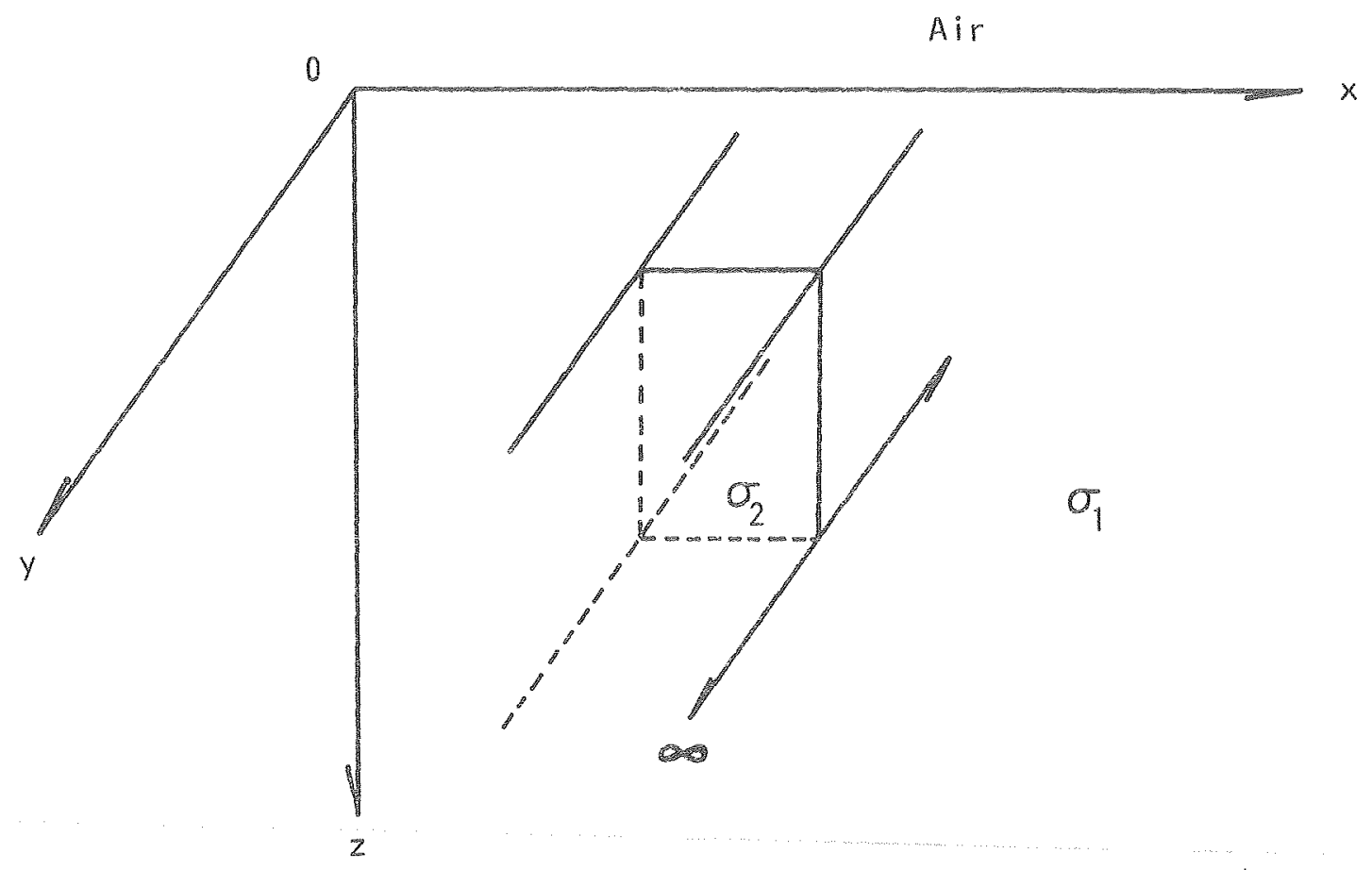

Figure 2. A two-dimensional geophysical electromagnetic system in which a magnetic dipole is located on or above the earth surface

XBL $789-10946$ 
Carrying out integration along y from $-L$ to $L$, we can reduce equation (II-15) into a much simplified two-dimensional form. Because of the orthogonality characterizing Fourier series, all but the squared terms disappear after integration. For the squared terms, we have

$$
\int_{-L}^{L}\left(\cos ^{2} \eta_{i} y, \sin ^{2} \eta_{i} y\right) d y=L, \eta_{i} \neq 0 .
$$

Hence, the variational integral becomes

$$
I(E)=I_{0}\left(\bar{E}_{0}\right)+\sum_{i=1}^{N} I_{i}\left(\bar{E}_{i}\right)
$$

where

$$
\begin{aligned}
I_{i}\left(E_{i}\right)=\frac{1}{L} \int_{s} & {\left[\frac{\rho^{2}}{2 \omega^{2} \mu}\left(-E_{x}^{2}+E_{y}^{2}-E_{z}^{2}\right)\right.} \\
& -\frac{1}{2 \omega^{2} \mu}\left\{\left(j \eta_{i} E_{z}-\frac{\partial E_{y}}{\partial z}\right)^{2}-\left(\frac{\partial E_{x}}{\partial z}-\frac{\partial E_{z}}{\partial x}\right)^{2}+\left(\frac{\partial E_{y}}{\partial x}-j \eta_{i} E_{x}\right)^{2}\right\} \\
& \left.+\frac{1}{J \omega}\left(-E_{x} J_{x}+E_{y} J_{y}-E_{z} J_{y}\right)\right] d x d z,
\end{aligned}
$$

and $I_{0}\left(E_{0}\right)$ is the zero harmonic variational integral, in which the electric field is polarized only in the direction parallel to the strike.

\section{B. Derivation of the wave equation for the secondary field}

The error contained in the total field numerical solution may be decomposed into two parts; the one carried by the primary field solution and the other by the secondary field solution. If we know the primary field solution analytically, the unnecessarily added error associated with the primary field numerical solution can be eliminated. Properly manipulating the system matrix equation, Coggon (1971) has demonstrated the advantage of the scheme in which the solution is sought in terms of the secondary field. As has been mentioned in the previous section, 
solving for the secondary field becomes not only useful but necessary in a situation where the original source is a magnetic dipole shown by (II-8). Even though the existence of the volume integral of the derivative of the delta function is mathematically guaranteed, it is numerically dangerous as far as the quality of the solution is concerned.

From the principle of superposition,

$$
\bar{E}=\bar{E}^{P}+\bar{E}^{s} \text {, }
$$

where the superscripts $p$ and s denote the primary and secondary fields

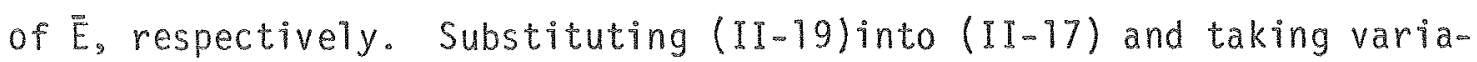
tions with respect to $\tilde{E}_{l}^{S}, l=1,2, \ldots, N$, with

$$
\delta \vec{E}=\delta \bar{E}^{s}
$$

and following Hildebrand (1965), in which

$$
\begin{aligned}
& \delta \int_{x_{1}}^{x_{2}} F\left(u, \frac{\partial u}{\partial x}\right) d x \\
& =\int_{x_{1}}^{x_{2}}\left\{\frac{\partial F}{\partial u}-\frac{\partial}{\partial x} \frac{\partial F}{\partial\left(\frac{\partial u}{\partial x}\right)}\right\} \delta u d x+\left.\frac{\partial F}{\partial\left(\frac{\partial u}{\partial x}\right)} \delta u\right|_{x_{1}} ^{x_{2}},
\end{aligned}
$$

we obtain the following $N$ independent sets of equations:

$$
\begin{aligned}
& \frac{1}{L} \int_{s} \frac{1}{\omega^{2} \mu}\left(-F_{x} \delta E_{x}^{s}+F_{y} \delta E_{y}^{s}-F_{z} \delta E_{z}^{s}\right) d x d z \\
& +\left.\frac{1}{L} \int_{x} \frac{1}{\omega^{2} \mu}\left\{\left(\frac{\partial E_{x}}{\partial z}-\frac{\partial E_{z}}{\partial x}\right) \delta E_{x}^{s}+\left(j \eta_{x} E_{z}-\frac{\partial E_{y}}{\partial z}\right) \delta E_{y}^{s}\right\}\right|_{z} d x \\
& -\left.\frac{1}{L} \int_{z} \frac{1}{\omega^{2} \mu}\left\{\left(\frac{\partial E_{y}}{\partial x}-j \eta_{h} E_{x}\right) \delta E_{x}^{s}+\left(\frac{\partial E_{x}}{\partial z}-\frac{\partial E_{z}}{\partial x}\right) \delta E_{z}^{s}\right\}\right|_{x} d z \equiv 0,
\end{aligned}
$$

where

$$
\begin{aligned}
& F_{x}=\left(k^{2}-\eta_{e}^{2}\right) E_{x}-j \eta_{k} \frac{\partial E_{y}}{\partial x}+\frac{\partial}{\partial z}\left(\frac{\partial E_{x}}{\partial z}-\frac{\partial E_{z}}{\partial x}\right)-j \omega \mu J_{x}, \\
& F_{y}=k^{2} E_{y}-j \eta_{l}\left(\frac{\partial E_{x}}{\partial x}+\frac{\partial E_{z}}{\partial z}\right)+\left(\frac{\partial}{\partial x^{2}}+\frac{\partial^{2}}{\partial z^{2}}\right) E_{y}-j \omega \mu J_{y}, \\
& F_{z}=\left(k^{2}-\eta_{l}^{2}\right) E_{z}-j \eta_{k} \frac{\partial E_{y}}{\partial z}-\frac{\partial}{\partial x}\left(\frac{\partial E_{x}}{\partial z}-\frac{\partial E_{z}}{\partial x}\right)-j \omega \mu J_{z} .
\end{aligned}
$$


line encircling the cross section, the last two line integrals in equation (II-22) will disappear. Depending upon the choice of the primary field, the secondary field would be either the total field minus the free space dipolar field or the scattered field caused oniy by lateral inhomogeneities. In either case it has been assumed that the effect of the central conductivity variations is negligible at the external bound$\operatorname{ary}$.

Since the variations of the three components of the secondary field $\vec{E}^{S}$ are independently arbitrary within the cross section $S$, their coefficients $F_{x}, F_{y}$ and $F_{z}$ must vanish identically. Hence, we have the following Euler equations for each $l, l=1,2, \ldots, N$ :

$$
F_{x}=F_{y}=F_{z}=0 \text {. }
$$

The last result is the same as the vector wave equation (I-12) provided that $\frac{\partial}{\partial y}$ and $\frac{\partial^{2}}{\partial y^{2}}$ substituted by $j k k_{y}$ and $-k_{y^{2}}^{2}$ respectively. This conforms that the solution obtained using variational integral is equivalent to that directly obtained from wave equation.

The solutions for the last three equations given by (II-26) have been in terms of three components of the total field $\bar{E}$. If we know the primary field $\overrightarrow{\mathrm{E}}^{\mathrm{p}}$, usually the free space dipolar field or the field in the presence of a layered half space, we can eliminate the source term in the following way. Let us write

$$
k^{2}=k_{p}^{2}+k_{s}^{2}
$$

where $k_{p}$ is the propagation constant of the primary field in the medium properly chosen. Then we can rewrite the first equation of (II-26) as

$$
\left(k_{p}^{2}+k_{s}^{2}-\eta_{l}^{2}\right)\left(E_{x}^{p}+E_{x}^{s}\right)-j \eta_{l} \frac{\partial}{\partial x}\left(E_{y}^{p}+E_{y}^{s}\right)
$$




$$
\begin{aligned}
& +\frac{\partial}{\partial z}\left\{\frac{\partial}{\partial y}\left(E_{x}^{p}+E_{x}^{s}\right)-\frac{\partial}{\partial x}\left(E_{z}^{p}+E_{z}^{s}\right)\right\}-j \omega \mu J_{x} \\
& =0 .
\end{aligned}
$$

The $x$-component scalar wave equation for the primary field can simply be transcribed from the vector wave equation (I.12). For $k_{y}=\eta_{2}$

$$
\left(k_{p}^{2}-\eta_{R}^{2}\right) E_{x}^{P}-j \eta_{l} \frac{\partial E_{y}^{P}}{\partial x}+\frac{\partial}{\partial z}\left(\frac{\partial E_{x}^{p}}{\partial z}-\frac{\partial E_{z}^{p}}{\partial x}\right)-j \omega_{\mu} J_{x}=0
$$

Subtracting (II-29) from (II-28), we obtain

$$
\left(k^{2}-\eta_{k}^{2}\right) E_{x}^{s}-j \eta_{l} \frac{\partial E_{y}^{s}}{\partial x}+\frac{\partial}{\partial z}\left(\frac{\partial E_{x}^{s}}{\partial z}-\frac{\partial E_{z}^{s}}{\partial x}\right)=-k_{z}^{2} E_{x}^{p} .
$$

Similarly,

$$
\begin{aligned}
& k^{2} E_{y}^{5}-j \eta\left(\frac{\partial E_{x}^{5}}{\partial x}+\frac{\partial E_{z}^{5}}{\partial z}\right)+\left(\frac{\partial^{2}}{\partial x^{2}}+\frac{\partial z^{2}}{\partial z^{2}}\right) E_{y}^{5}=-k_{y}^{2} E_{y}^{p}, \\
& \left(k^{2}-\eta_{k}^{x}\right) E_{3}^{5}-j \eta_{2} \frac{\partial E_{y}^{5}}{\partial z}-\frac{\partial}{\partial x}\left(\frac{\partial E_{x}^{5}}{\partial z}-\frac{\partial E^{5}}{\partial x}\right)=-k_{s}^{2} E_{3}^{p} .
\end{aligned}
$$

The last three equations are coupled inhomogeneous scalar wave equations in terms of the secondary fields $E_{x}^{S}, E_{y}^{S}$, and $E_{z}^{S}$. The original source $j w / J_{s}$ has been eliminated from the system and substituted by $=k_{s}^{2} E^{p}$.

The physical interpretation for these equations can be made using the concept of the scattering current (Harrington, 1967). Upon being combined together, with $i \eta_{2}$ and $-\eta_{z}^{2}$ substituted by $\frac{\partial}{\partial y}$ and $\frac{\partial^{2}}{\partial y^{2}}$, respec. tively, these equations can be rewritten as

$$
k^{2} E^{S}-\nabla \times \nabla \times E^{S}=-k_{s}^{2} E^{P} .
$$

Supposing that

$$
k_{p}^{2}=\omega^{2} \mu \varepsilon-j \sigma_{p} \omega \mu \text {, }
$$

and

$$
k_{s}^{2}=-j \omega \mu\left(\sigma-\sigma_{p}\right),
$$

then equation (II-33) can be manipulated into

$$
k_{p}^{2} \bar{E}^{s}-\nabla \times \nabla \times \bar{E}^{s}=-j \omega \mu \bar{J}^{s},
$$


where the scattering current $j^{5}$ is defined by

$$
\bar{J}^{s}=\left(\sigma-\sigma_{p}\right) \bar{E} \text {. }
$$

The integral equation solution for equation (II-36) was given by Harrington (1961) as

$$
\bar{E}^{s}(\vec{r})=\int_{v} \overline{\bar{r}}\left(\bar{r} ; \bar{r}^{\prime}\right) \cdot \bar{J}^{s}\left(\bar{r}^{\prime}\right) d v^{\prime}
$$

where $\overline{\vec{r}}\left(\bar{r}_{;}, \bar{r}^{\prime}\right)$ is a tensor Green's function relating $\bar{E}^{S}$ at $\bar{r}$ to an element of current $\bar{J}^{5}$ at $\bar{r}^{2}$. Solutions using this approach have been found by Hohmann (1971 \& 1975), Meyer (1977), and Lee (1978) following their own particular geophysical interests. 


\section{Field Approximation}

A. Finite element method

The basic equation, a two-dimensional variational integral, has been derived in the previous section by transforming out the strike directional dependence using Fourier series expensions, Rewriting equation $(11-18)$ for $\eta_{i}=k_{y^{2}}$

$$
\begin{aligned}
I\left\{E\left(k_{y}\right)\right\} & =\frac{1}{L} \int_{s}\left[\frac{k^{2}}{2 \omega^{2} \mu}\left(-E_{x}^{2}+E_{y}^{2}-E_{z}^{2}\right)\right. \\
& -\frac{1}{2 \omega^{2} \mu}\left\{\left(j k_{y} E_{z}-\frac{\partial E_{y}}{\partial z}\right)^{2}-\left(\frac{\partial E_{x}}{\partial z}-\frac{\partial E_{z}}{\partial x}\right)^{2}+\left(\frac{\partial E_{y}}{\partial x}-j k_{y} E_{x}\right)^{2}\right\} \\
& \left.+\frac{1}{J \omega}\left(-E_{x} J_{x}+E_{y} J_{y}-E_{z} J_{z}\right)\right] d x d z .
\end{aligned}
$$

The approximation to integral (III-1) can be made using a finite element technique. The idea is that the integral can be approximately evaluated in terms of a finite number of unknowns by forcing the localized field to behave in a simple but physically acceptable way. As a result, the integral, upon being integrated, will become the sum of property weighted discretized unknown electric fields in a quadratic form. Following the variational principle, which is equivalent to the least square criterion in this case, a system of linear equations will be generated.

Throughout the formulation process a rectangle has been used as the fundamental element. The prescribed base function interpolates electric fields bi-linearly in $x-z$ plane. The continuity of electric field is preserved across cell boundaries by modification of the original step like conductivity structure to a continuous one (Appendix II). Let us write the field $f$ defined within a rectangle (Figure 3 ) as

$$
f=a+b x+c z+d x_{z} .
$$


The coefficients $a, b, c$, and d are functions of fixed positions $\left(x_{i}, z_{j}\right)$, $i, j=1 \sim 4$, and of the unknown fields $f_{i}, i=1 \sim 4$, designated at four nodal points. Using shape function N (Zienkiewicz, 1971), f may be conveniently rewritten in a matrix form as

$$
t=N^{T} \cdot \underline{t}
$$

where "-" indicates a matrix and

$$
\begin{aligned}
& \underline{t}^{T}=\left(\begin{array}{llll}
t_{1} & f_{2} & f_{3} & f_{4}
\end{array}\right), \\
& \underline{N}^{\top}=\left(\begin{array}{llll}
N_{1} & N_{2} & N_{3} & N_{4}
\end{array}\right)=\left(\begin{array}{cccc}
1 & x & z & x_{3}
\end{array}\right) \cdot A, \\
& A=\frac{1}{\Delta}\left|\begin{array}{cccc}
x_{2} z_{3} & -x_{1} z_{3} & x_{1} z_{1} & -x_{2} z_{1} \\
-z_{3} & z_{3} & -z_{1} & z_{1} \\
-x_{2} & x_{1} & -x_{1} & x_{2} \\
1 & -1 & 1 & -1
\end{array}\right|
\end{aligned}
$$

" $\Delta$ " is the area of the rectangle and " $T$ " indicates the transpose of a matrix. The derivatives of $f$ can be easily found from (III-4) as

$$
\begin{aligned}
& \frac{\partial t}{\partial x}=\frac{\partial}{\partial x} \underline{N}^{T} \underline{t}=\left(\begin{array}{llll}
0 & 1 & 0 & z
\end{array}\right) \cdot A \cdot I \\
& =\left(\begin{array}{llll}
c_{1} & c_{2} & c_{3} & c_{4}
\end{array}\right) \cdot \frac{1}{2}, \\
& \frac{\partial f}{\partial z}=\frac{\partial}{\partial z} N^{T} \cdot \underline{f}=\left(\begin{array}{llll}
0 & 0 & (x
\end{array}\right) \cdot A \cdot \underline{t} \\
& =\left(\begin{array}{llll}
D_{1} & D_{2} & D_{3} & D_{4}
\end{array}\right) \cdot \frac{1}{2} \text {, }
\end{aligned}
$$

where $C_{i}, i=1 \sim 4$, and $D_{j}, j=1 \sim 4$, are linear functions of $z$, and functions of $x$, respectively.

Before we proceed to the next step, let us reduce the variational integral (III-I) so that the variation can be directly taken with respect to the secondary electric field $\vec{E}^{5}$. Using $(I I-19)$ and $(I I-27)$, we write

$$
I\left\{\bar{E}\left(k_{y}\right)\right\}=I\left\{\bar{E}^{P}\left(k_{y}\right)\right\}+I\left\{\bar{E}^{s}\left(k_{y}\right)\right\}+P\left\{\bar{E}^{P}\left(k_{y}\right), \bar{E}^{s}\left(k_{y}\right)\right\},
$$




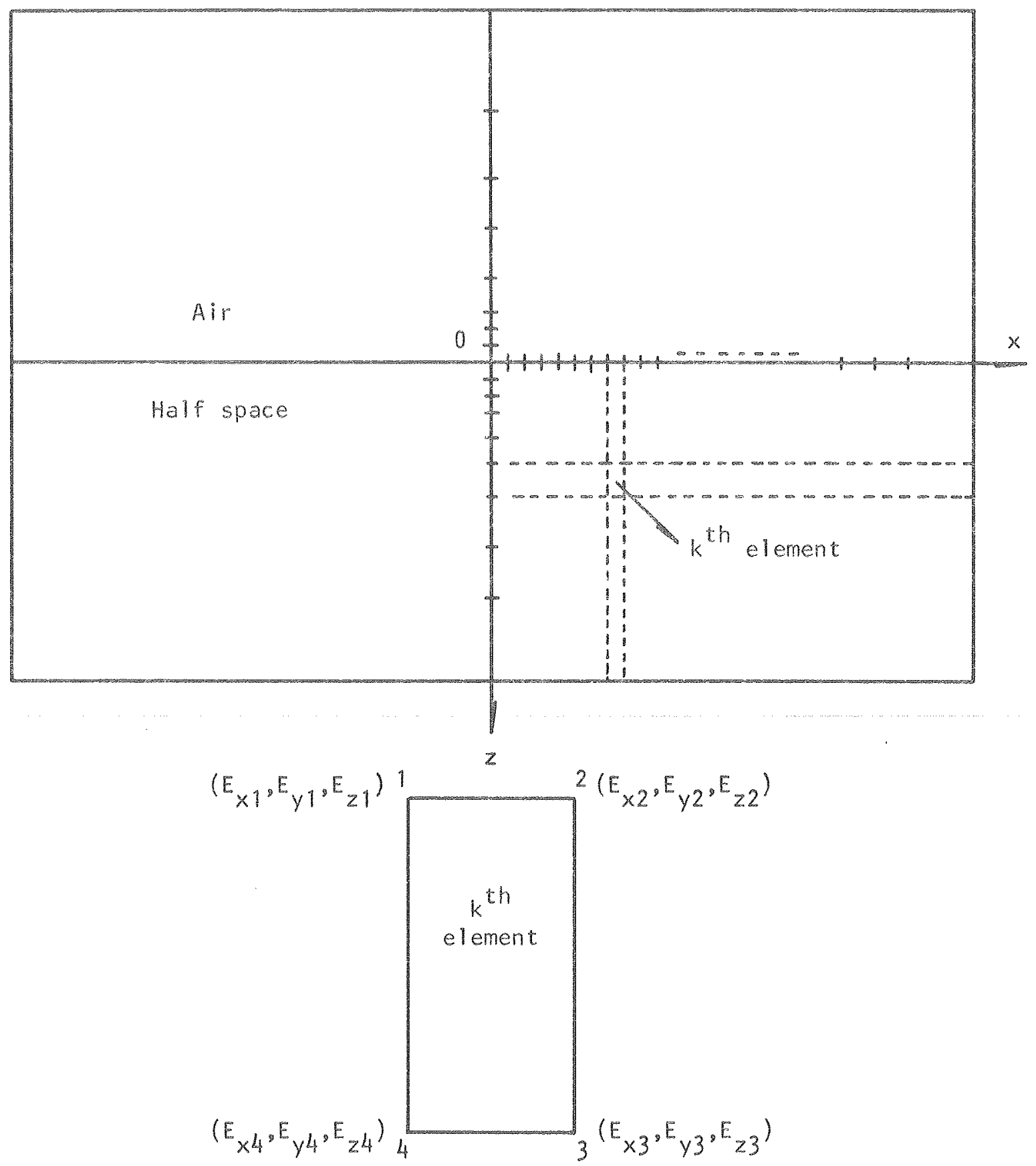

Figure 3. A finite element grid structure and notations used for a rectangle 
where

$$
\begin{aligned}
& P\left\{\bar{E}^{P}\left(k_{y}\right), \bar{E}^{s}\left(k_{y}\right)\right\}=\frac{1}{L} \int_{s}\left[\frac{k_{s}^{2}}{\omega^{2} \mu}\left(-E_{x}^{s} E_{x}^{P}+E_{y}^{s} E_{y}^{P}-E_{z}^{s} E_{z}^{P}\right)\right. \\
& -\frac{1}{\omega^{2} \mu}\left\{\left(j k_{y} E_{z}^{s}-\frac{\partial E_{y}^{s}}{\partial z}\right)\left(j k_{y} E_{z}^{P}-\frac{\partial E_{y}^{P}}{\partial z}\right)-\left(\frac{\partial E_{x}^{S}}{\partial z}-\frac{\partial E_{z}^{s}}{\partial x}\right)\left(\frac{\partial E_{x}^{p}}{\partial z}-\frac{\partial E_{z}^{p}}{\partial x}\right)\right. \\
& \left.+\left(\frac{\partial E_{y}^{s}}{\partial x}-j k_{y} E_{x}^{s}\right)\left(\frac{\partial E_{y}^{p}}{\partial x}-j k_{y} E_{x}^{p}\right)\right\} \\
& \left.+\frac{\left.\right|_{p} ^{2}}{\omega^{2} \mu}\left(-E_{x}^{s} E_{x}^{p}+E_{y}^{s} E_{y}^{p}-E_{y}^{s} E_{z}^{p}\right)\right] d x d z \text {. }
\end{aligned}
$$

Integrating those derivatives of the secondary electric fields by parts, we can rewrite (III-7) as

$$
\begin{aligned}
& I\left\{\vec{E}\left(k_{y}\right)\right\}=I\left\{\vec{E}^{P}\left(k_{y}\right)\right\}+Q \\
& \left.+\frac{1}{L}\right)_{s}\left[\frac{k^{2}}{2 w^{2} \mu}\left(-E_{x}^{s^{2}}+E_{y}^{s^{2}}-E_{z}^{s^{2}}\right)-\frac{1}{2 \omega^{2} \mu}\left\{\left(j k_{y} E_{z}^{s}-\frac{\partial E_{y}^{s}}{\partial z}\right)^{2}\right.\right. \\
& \left.-\left(\frac{\partial E_{x}^{s}}{\partial z}-\frac{\partial E_{z}^{s}}{\partial x}\right)^{2}+\left(\frac{\partial E_{y}^{s}}{\partial x}-j k_{y} E_{x}^{s}\right)^{2}\right\} \\
& \left.+\frac{k_{s}^{2}}{w^{2} \mu}\left(-E_{x}^{S} E_{x}^{p}+E_{y}^{s} E_{y}^{p}-E_{z}^{s} E_{z}^{p}\right)\right] d x d z,
\end{aligned}
$$

where

$$
\begin{aligned}
& Q=\left.\frac{1}{L} \int_{x} \frac{1}{\omega^{2} \mu}\left\{\left(\frac{\partial E_{x}^{p}}{\partial z}-\frac{\partial E_{z}^{p}}{\partial x}\right) E_{x}^{s}+\left(j k_{y} E_{z}^{p}-\frac{\partial E_{y}^{p}}{\partial z}\right) E_{y}^{s}\right\}\right|_{y} d x \\
& -\left.\frac{1}{L} \int_{z} \frac{1}{\omega^{2} \mu}\left\{\left(\frac{\partial E_{y}^{p}}{\partial x}-j k_{y} E_{x}^{p}\right) E_{y}^{s}+\left(\frac{\partial E_{x}^{p}}{\partial z}-\frac{\partial E_{z}^{p}}{\partial x}\right) E_{z}^{s}\right\}\right|_{x} d z \\
& +\frac{1}{L} \int_{S} \frac{1}{\omega^{2} \mu}\left[-E_{x}^{s}\left\{\left(k_{p}^{x}-k_{y}^{2}\right) E_{x}^{p}-j k_{y} \frac{\partial E_{y}^{p}}{\partial x}+\frac{\partial}{\partial z}\left(\frac{\partial E_{x}^{p}}{\partial z}-\frac{\partial E_{x}^{p}}{\partial x}\right)-j \omega_{\mu} J_{x}\right\}\right. \\
& +E_{y}^{s}\left\{k_{p}^{z} E_{y}^{p}-j k_{y}\left(\frac{\partial E_{x}^{p}}{\partial x}+\frac{\partial E_{z}^{p}}{\partial z}\right)+\left(\frac{\partial^{2}}{\partial x^{2}}+\frac{\partial z}{\partial z^{z}}\right) E_{y}^{p}-j \omega \mu J_{y}\right\} \\
& \left.-E_{z}^{s}\left\{\left(k_{p}^{x}-k_{y}^{x}\right) E_{z}^{P}-j k_{y} \frac{\partial E_{y}^{P}}{\partial z}-\frac{\partial}{\partial x}\left(\frac{\partial E_{x}^{P}}{\partial z}-\frac{\partial E_{z}^{p}}{\partial x}\right)-j \omega \mu_{3} J_{z}\right\}\right] d x d z \text {. }
\end{aligned}
$$

The third integral in (III-10) vanishes since the coefficients of $E_{x}^{5}$, $E_{y}^{S}$, and $E_{z}^{S}$ are the three scalar entities of the primary field vector wave equation shown by equation (I-12). At each internal cell boundary the first and the second integrals of (III-10) also cancel out analytically between adjacent cells. Therefore Q becomes a constant composed of the line integrals evaluated at external boundaries where both the 
primary and the secondary electric fields are known. Since the variation of a constant is zero, the effective variational integral becomes the integral part of equation (1/1-9), which is

$$
\begin{aligned}
& \left.\widetilde{I} \mid E^{s}\left(l_{y}\right)\right\}=\frac{1}{L} \int_{S}\left[\frac{k^{2}}{2 h^{2} \mu}\left(-E_{x}^{s^{2}}+E_{y}^{s^{2}}-E_{z}^{s^{2}}\right)\right. \\
& -\frac{1}{2 \omega^{2} \mu}\left\{\left(j k_{y} E_{z}^{s}-\frac{\partial E_{y}^{s}}{\partial z}\right)^{2}-\left(\frac{\partial E^{5}}{\partial z}-\frac{\partial E^{s}}{\partial x}\right)^{2}+\left(\frac{\partial E_{y}^{s}}{\partial x}-j k_{y} E_{x}^{s}\right)^{2}\right\} \\
& \left.+\frac{k_{s}^{2}}{\omega^{2} \mu}\left(-E_{x}^{s} E_{x}^{p}+E_{y}^{s} E_{y}^{p}-E_{z}^{s} E_{z}^{p}\right)\right] d x d y \text {. }
\end{aligned}
$$

The elementary matrix representing the last equation can be readily shown as

$$
\widetilde{I}^{2}\left(E^{3}\right)=\frac{1}{2} E^{S T} \cdot K^{8} \cdot E^{S}+E^{S T} \cdot K_{s} \cdot E^{P}
$$

where the superscript e indicates elementary and

$$
E^{\top}=\left(E_{x 1} E_{y 1} E_{31} E_{x 2} E_{y 2} E_{32} E_{x 3} E_{y 3} E_{33} E_{x 4} E_{y 4} E_{y_{4}}\right) .
$$

The elementary $(12 \times 12)$ system matrix $K^{e}$ and the source matrix $k_{-s}^{e}$ are given by

$$
\begin{aligned}
& K_{s}^{e}=\frac{1}{L} \int_{\Delta}\left(\frac{k^{2}}{w^{2} \mu} K_{1}^{e}-\frac{1}{\omega^{2} \mu} K_{z}^{e}\right) d x d z, \\
& K_{s}^{e}=\frac{1}{L} \int_{\Delta} \frac{K_{s}^{2}}{w^{2} \mu} K_{i}^{e} d x d z,
\end{aligned}
$$

where the variable matrices $K_{-1}^{e}$ and $K_{2}^{e}$ are given as follows:

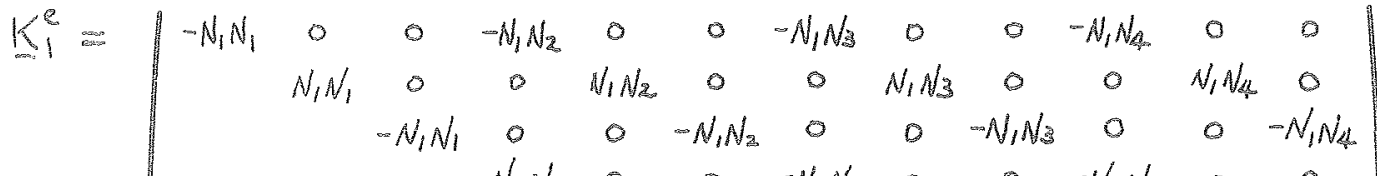

$$
\begin{aligned}
& \begin{array}{llllllllll}
-N_{2} N_{2} & 0 & 0 & -N_{2} N_{3} & 0 & 0 & -N_{2} N_{4} & 0 & 0
\end{array} \\
& N_{2} N_{2} \circ 0 N_{2} N_{3} \circ 0 \quad N_{2} N_{4} \quad 0 \\
& \begin{array}{lllllll}
-N_{2} N_{2} & 0 & 0 & -N_{2} N_{3} & 0 & 0 & -N_{2} N_{4}
\end{array} \\
& \text { Symmetric } \\
& \begin{array}{cccccc}
-N_{3} N_{3} & 0 & 0 & -N_{3} N_{4} & 0 & 0 \\
& N_{3} N_{3} & 0 & 0 & N_{3} N_{4} & 0
\end{array} \\
& -N_{3} N_{3} 000-N_{3} N_{4} \\
& -N_{4} N_{4} O \quad 0 \\
& N_{4} N_{4} \quad 0 \\
& -N_{4} N_{4}
\end{aligned}
$$




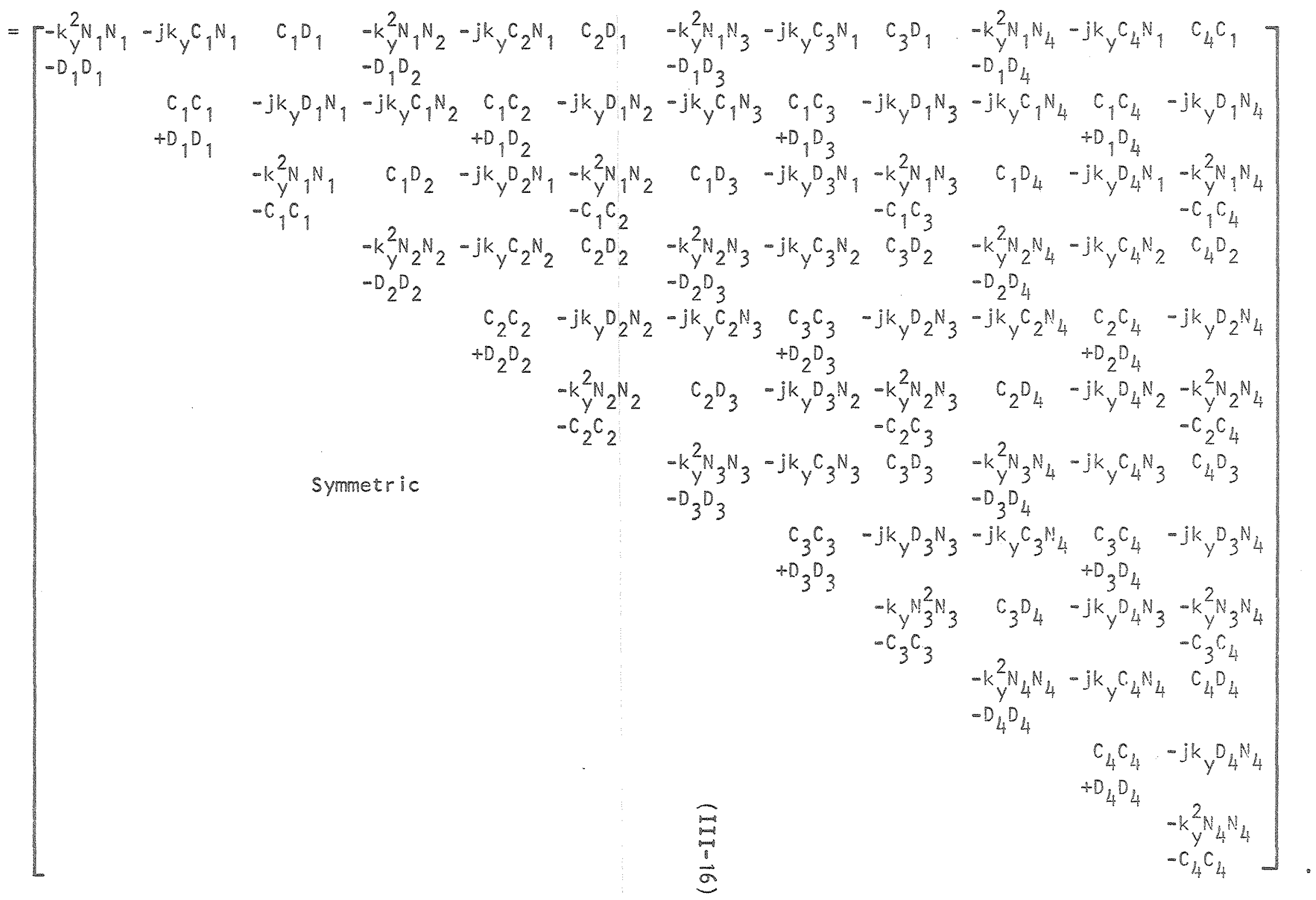

$\cong$ 
After integration over the rectangular area (refer to the Table 1), the elementary system matrix $\underline{K}^{e}$ is stored in the cotal system matrix $K$. The elementary source vector is a column matrix obtained by multiplying the source matrix $K_{s}^{e}$ by the primary electric field $E^{p}$. After all the elementary matrices are added together, the matrix representation of the total variational integral becomes

$$
\tilde{I}\left(E^{s}\right)=\sum_{e} \tilde{I} e\left(E^{5}\right)=\frac{1}{2} E^{s} \cdot K \cdot E^{s}+E^{S}{ }^{T} \underline{B}^{p},
$$

where the superscript $p$ indicates that the source vector is originated from the primary field. The total system matrix $K$ is sparse, banded and symetric, not necessarily diagonally dominant but positive definite.

Following the variational principle, the condition by which the variational integral (III-17) becomes stationary is that:

$$
\sigma \tilde{I}\left(E_{\infty}^{s}\right) \equiv 0
$$

The last equation generates a set of simultaneous Tinear equations

$$
K \underline{E}^{5}+\underline{B}^{P}=0
$$

This is the basic equation from which the secondary electric field will be solved. The unique solution however cannot be obtained at this stage without imposing the properly chosen boundary conditions (Stratton, 1941).

\section{B. Boundary condition}

In a brief discussion in part $I I$, we have been able to derive wave equations by forcing the line integrals written in a variational form to vanish (refer to equation (II-22)). There are two distinctive conditions available. These conditions are:

(1) the secondary tangential electric fields are prescribed on the line $l$ encircling $S$, so that

$$
\bar{n} \times\left.\delta \bar{E}^{s}\right|_{0}=0
$$


Table 1

Table of integrations for the evaluations of $\underline{K}^{e}$ and $\underline{K}_{s}^{e}$.

$$
F=\int_{z_{2}}^{z_{3}} \int_{x_{1}}^{x_{2}} f(x, z) d x d z
$$

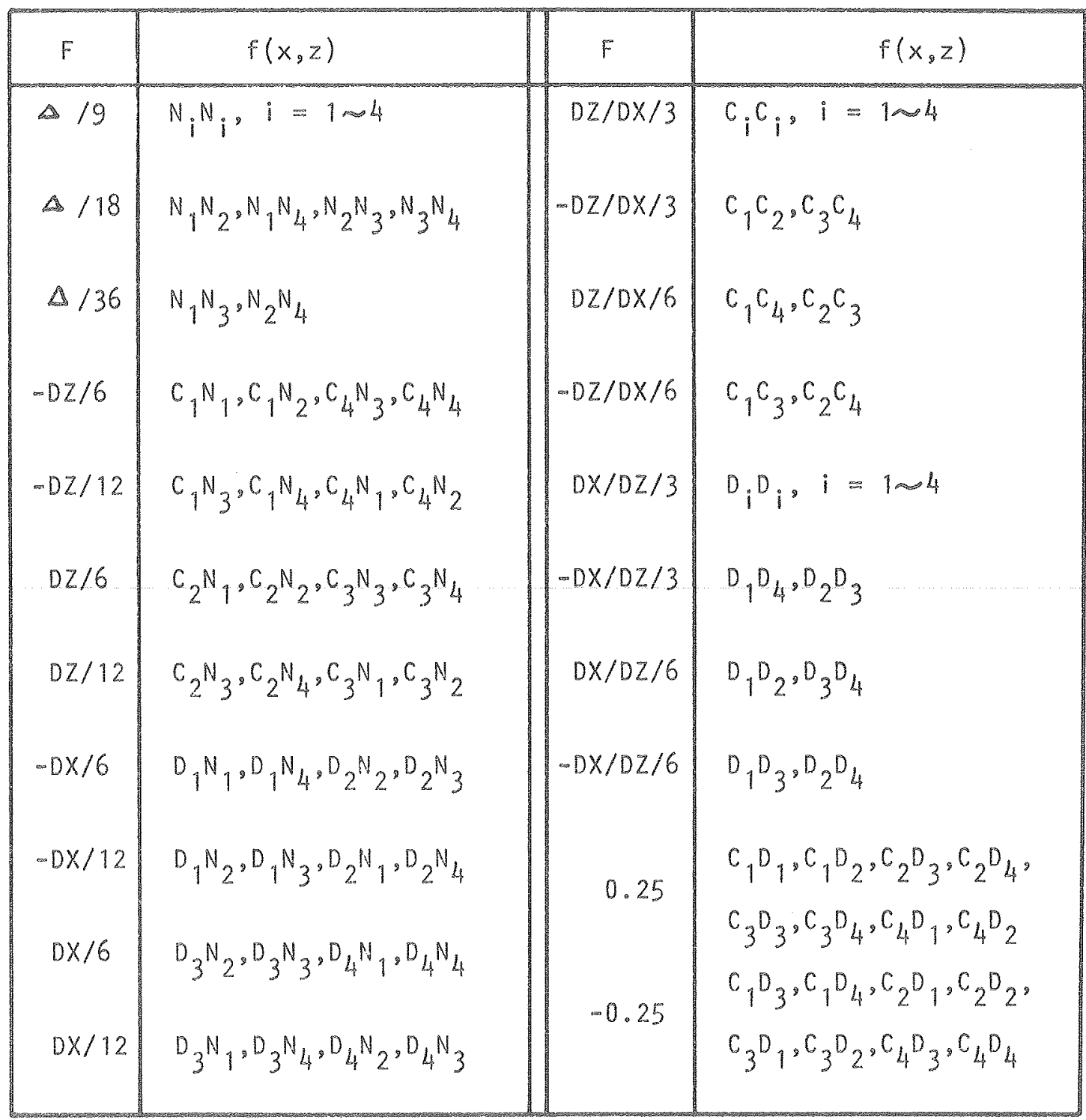

$$
\begin{aligned}
& C_{i}=N_{i} / 2 x, \quad D_{i}=N_{i} / 2 ; \quad i=1 \approx 4 \\
& D X=x_{2}-x_{1}, D Z=z_{3}-z_{2}, \quad \text { and } \Delta=D X \cdot D Z
\end{aligned}
$$


(2) tangential magnetic fields are zero, that is

$$
\bar{n} \times\left.\bar{H}\right|_{Q}=0 \text {. }
$$

Condition (2) would be the case in which the boundary material was a perfect magnetic conductor. Under this condition, the resultant tangential component of electric field, nxE, would be exactly doubled at the boundary due to perfect reflection. The same condition could be simulated using the method of images. Although this is rarely a realistic condition, the concept can be made use of in conjunction with the complementary condition where the boundary material is a perfect electric conductor, so that the opposite phenomena would take place (see Babinet's principle; Jackson, 1967). In practice, however, this is not the economical way of approaching the problem, for the scheme would require too many computations in order to annihilate the various order of reflections expected to occur along the closed boundary.

Throughout this study, we have used boundary condition (1), a common Dirichlet type boundary condition. With the secondary electric fields prescribed at the boundary, equation (III-19) can be partitioned into

$$
\left|\begin{array}{ll}
K_{i i} & K_{i b} \\
K_{b i} & K_{b b}
\end{array}\right| \cdot\left|\begin{array}{l}
E_{i}^{s} \\
E_{b}^{s}
\end{array}\right|=-\left|\begin{array}{c}
B_{i}^{p} \\
B_{b}^{p}
\end{array}\right|,
$$

where the subscript $i$ indicates that the variable attached to it is defined inside the boundary, and the subscript b at the boundary. Since $E_{b}^{s}$ is known, the equation can be reduced into

$$
K_{i i} \cdot \underline{E}_{i}^{S}=\underline{B},
$$


where the resultant source vector $B$ is

$$
B=-B_{i}^{p}-K_{i b} \cdot E_{b} .
$$

The secondary electric field obtained by solving equation (III-23) must be unique by definition. The error associated with the solution is strictiy subject to the degree of accuracy implemented in the process of discretizing the variational integral.

\section{Source vector}

In formulating the resultant source vector $B$, we have found that it is necessary to know not only the secondary electric fields along the external boundary but the primary fields at every nodal point in the inhomogeneous region. The free space dipolar field has been used for the primary field. One of the major advantages of the scheme is that it only requires the calculation of Bessel functions such as $K_{0}$ and $\mathrm{K}_{\mathrm{q}}$.

The secondary electric field at the boundary is the total field subtracted by the free space dipolar field. The total electric field in $k_{y}$ harmonic space can be obtained by Fourier transforming the solution obtained for a layered half-space. The layered half-space responses due to a horizontal magnetic dipole and a horizontal loop have been given by Dey and Ward (1970) and Ryu et al (1970), respectively. The Fourier transformation approach however, would entail the following technical problems:

(1) a large number of field point should be accounted for in order to retain the highest possible harmonic component.

(2) it would be difficult to compute boundary values along the top and the bottom boundaries in harmnnic space if the side boundaries 
is necessary.

In an effort to ease these problems, we have developed a direct solution in harmonic space using a Hertz potential of the magnetic type. The magnetic Hertz potential $\prod^{*}$ satisfies

$$
\nabla^{2} \pi^{*}+k^{2} \pi^{*}=-\bar{M}_{s}
$$

where

$$
\vec{M}_{s}=\bar{m}_{5} \delta(z-x) \delta(y) \delta(z-y)
$$

The electromagnetic field vectors have been deduced (Sommerfeld. 1926) from the Hertz potential il as

$$
\begin{aligned}
& E=-j \omega \mu \nabla \times \pi^{*}, \\
& \bar{H}=k^{2} \pi^{*}+\nabla\left(\nabla \cdot \bar{\pi}^{*}\right) .
\end{aligned}
$$

It can be shown (Appendix III) that the particular, or the primary, solution of equation (III-25) in $\mathrm{K}_{\mathrm{y}}$ is

$$
\begin{aligned}
\pi^{* P}\left(x, k_{y}, z\right) & =\frac{\bar{m}_{5}}{2-\pi} \int_{0}^{\infty} \frac{e^{-u\left|3-z^{\prime}\right|} \cos k_{x}(x-x) d k_{x}}{u} \\
& =\frac{m_{s}}{2 \pi} k_{0}(\gamma r),
\end{aligned}
$$

Where $k_{0}$ is the modified Bessel function of the second $k$ ind of order zero and

$$
\begin{aligned}
& \gamma=\left(k_{y}^{2}-k^{2}\right)^{1 / 2} \\
& u=\left(k_{x}^{2}+k_{y}^{2}-k^{2}\right)^{1 / 2} \\
& r=\left\{\left(x-x^{2}\right)^{2}+\left(z-z^{2}\right)^{2}\right\}^{1 / 2}
\end{aligned}
$$

The necessary derivatives of $k_{0}(y r)$ for the evaluations of the prit mary electric and magnetic fields are: 


$$
\begin{aligned}
& \frac{\partial}{\partial x} K_{0}(y r)=-\frac{\gamma}{r}\left(x-x^{\prime}\right) K_{1}(y r), \\
& \frac{\partial}{\partial z} K_{0}(\gamma r)=-\frac{\gamma}{r}\left(z-z^{\prime}\right) K_{1}(\gamma r), \\
& \frac{\partial^{2}}{\partial x \partial z} K_{0}(\gamma r)=\frac{\gamma}{r^{2}}\left(x-x^{\prime}\right)\left(z-z^{\prime}\right)\left\{\gamma K_{0}(\gamma r)+\frac{z}{r} K_{1}(\gamma r)\right\}, \\
& \frac{\partial^{2}}{\partial x^{2}} K_{0}(\gamma r)=\frac{\gamma^{2}}{r^{2}}\left(x-x^{\prime}\right)^{2} K_{0}(\gamma r)+\frac{\gamma}{r^{3}}\left\{z\left(x-x^{\prime}\right)^{2}-r^{2}\right\} K_{1}(\gamma r), \\
& \frac{\partial^{2}}{\partial z^{2}} K_{0}(\gamma r)=\frac{\gamma^{2}}{r^{2}}\left(z-z^{\prime}\right)^{2} K_{0}(\gamma r)+\frac{y}{r^{3}}\left\{z\left(z-z^{\prime}\right)^{2}-r^{2}\right\} K_{1}(\gamma r) .
\end{aligned}
$$

The differential operators $\frac{\partial}{\partial y}$ and $\frac{\partial^{2}}{\partial y^{2}}$ have always been treated as constants $j k_{y}$ and $-k_{y}^{2}$ respectively.

The secondary potential $\bar{\pi}^{*^{s}}$ due to the presence of the layered half space is given by Appendix III. For the sake of simplicity, let us choose the secondary Hertz potential in the air in the presence of a vertical magnetic dipole. Rewriting the secondary part of equation (9) derived in Appendix III

$$
\left.\bar{\pi}_{z 0}^{x^{s}}=\frac{m_{3}}{2 \pi} \int_{0}^{+\infty} A_{0}^{-} e^{u_{0} y+u_{0} z^{\prime}} \cos k_{x}\left(x-x^{\prime}\right) d k_{x}\right)
$$

where $A_{0}^{-}$is the reflection coefficient found by matching boundary conditions.

Substituting (III-29) into (III-26), we find the secondary electric fields in the air as

$$
\begin{aligned}
& E_{x}^{s}=\omega \mu k_{y} \frac{m_{y}}{2 \pi} \int_{0}^{\infty} A_{0}^{-} e^{u_{0} z+u_{0} y^{\prime}} \cos k_{x}\left(x-x^{\prime}\right) d k_{x}, \\
& \left.E_{y}^{s}=-j \omega \mu \frac{m_{z}}{2 \pi} \int_{0}^{\infty} A_{0}^{-} e^{u_{y} z+u_{0} y^{\prime}} k_{x} \sin k_{x}\left(x-x^{\prime}\right) d k_{x}\right) \\
& E_{z}^{s}=0 .
\end{aligned}
$$

These secondary electric fields have been used for the boundary values in the air. 
If the model of interest has side boundaries of different half space structures, the secondary electric field along the top boundary may be approximated by the following interpolation scheme. Instead of interpolating the complex fields themselves, we would rather interpolate the reflection coefficients assuming that the value falls in the range bounded by two reflection coefficients; one obtained for the left side boundary $A_{l}^{-}$, and the other for the right side boundary, $A_{r}^{-}$. Needless to say, both side boundaries must be placed sufficiently far from the central region of the model, so that the reflection coefficients obtained for both layered half spaces may be effectively valid. The effective reflection coefficient, $A^{-}$, is approximated by a function of $x$ and the horizontal wave number $\lambda=\left(k_{x}^{2}+k_{y}^{2}\right)^{1 / 2}$, as

$$
A^{-}=\frac{1}{2}\left[\left\{1+\tanh \left(\frac{x}{D} \ln \frac{1-\alpha}{1+\alpha}\right)\right\} A_{R}^{-}+\left\{1+\tanh \left(\frac{x}{D} \ln \frac{1+\alpha}{1-\alpha}\right)\right\} A_{r}^{-}\right],
$$

where $D$ is the distance between the two side boundaries and $\alpha$ is a function of $\lambda$ arbitrarity defined as

$$
\alpha=0.95+0.05 \tanh (\lambda) .
$$

The interpolation function (III-33) represents a curve basically interpolating the two reflection coefficients $A_{2}^{-}$and $A_{r}^{-}$in the form of an exponential transition. The larger the horizontal wave number $\lambda$ becomes, the more rapid transition the reflection coefficient experiences along $x$ across the center $x=0$.

The secondary electric fields along the top boundary have been computed using the effective reflection coefficient, $A^{-}$, given by (III-33). The similar procedure has been taken for the computations of the boundary values along the bottom boundary. 
IV. Evaluation of the Numerical Solution

\section{A. Secondary field solution}

The secondary electric field for a given $k_{y}$ has been obtained by directly solving matrix equation (III-23) formulated in the previous section. The direct solution of (III-23) may generally be written as

$$
\underline{E}_{i}^{S}=K_{-i}^{-1} \cdot B \text {. }
$$

A complex version of one of the direct solution routines written by Reid (1972) has been used. The algorithm used for the inversion of $K_{i j}$, a square matrix of order $n$ with bandwidth $m$, is symmetric Cholesky Decomposition (Martin and Wilkinson, 1965). According to the theorem, the symmetric, positive definite matrix $K_{j i}$ has a unique lower triangular matrix $L$ of the same order and bandwidth such that

$$
L_{\infty}^{T}=K_{i \uparrow}
$$

Therefore the solution $E_{i}^{S}$ can alternately be found by

$$
E_{i}^{S}=\left(L^{T}\right)^{-1} \cdot\left(L^{-1} B\right)
$$

The routine uses random disc access facilities in transferring data from or to the working storage area. The decomposition process (IV-2) requires approximately $n \times(m+1) \times(m+2) / 2$ multiplications and $n$ square root operations of complex numbers. Once the triangular matrix L is found, each solution corresponds to an additional source vector $B$ requires only $2 \times n \times(m+1)$ multiplications in step (IV-3). It has been found that this method uses substantially less computing time than the matrix inversion scheme using a Gaussian elimination algorithm. For the solution of complex equations whose system matrix is of $n=1980$ and $m=76$, it takes 25 CPU seconds on the CDC 7600 at Lawrence Berkeley Laboratory using Gaussian elimination technique: 
whereas only 6 CPU seconds are required for the same result using the decomposition technique.

A mesh size of $55 \times 18$, Figure 3 , has been used for a 11 the models studied here. The system matrix $\underline{k}_{i j}$ is of order 2970 with its maximum half bandwidth of 60 . It takes 21 CPU seconds for a complete solution $\mathrm{E}^{S}\left(k_{y}\right)$ on the CDC 7600. Each solution for an additional source vector requires 5 CPU seconds including the computing time spent for the formulation of source vector itself.

The first test model is a uniform half space of 100 ohm-meter resistivity. The transmitter is located at 2 meters above the earth surface and a frequency of $25 \mathrm{kHz}$ is used.

The secondary electric fields $E_{x}^{s}\left(k_{y}\right)$ and $E_{y}^{S}\left(k_{y}\right)$ due to a vertical magnetic dipole of unit moment have been plotted in Figure 4 and Figure 5, respectively. The field point is located at the surface 18 meters away from the source to the right. Throughout the entire range of $k_{y}$ values the numerical solutions obtained are virtually identical to the analytic solutions. Although some errors are observed towards the end of $k_{y}$ axis, their contributions, after being inversely fourier transformed, will be insignificant.

With the same model the secondary electric fields $E_{x}^{S}\left(k_{y}\right)$ and $E_{y}^{S}\left(k_{y}\right)$ due to a horizontal magnetic dipole of unit moment have been calculated. The same frequency and source position have been used. Figure 6 and Figure 7 show the real and imaginary parts of $E_{x}^{5}\left(k_{y}\right)$ and $E_{y}^{S}\left(k_{y}\right)$ calculated at the same field point, respectively. The dominating parts, the real part of $E_{x}^{5}\left(k_{y}\right)$ in Figure 6 and the imaginary part of $E_{y}^{S}\left(k_{y}\right)$ in Figure 7 , deviate no more than a few percent from the analytic solutions. However, we have found substantial errors in the 


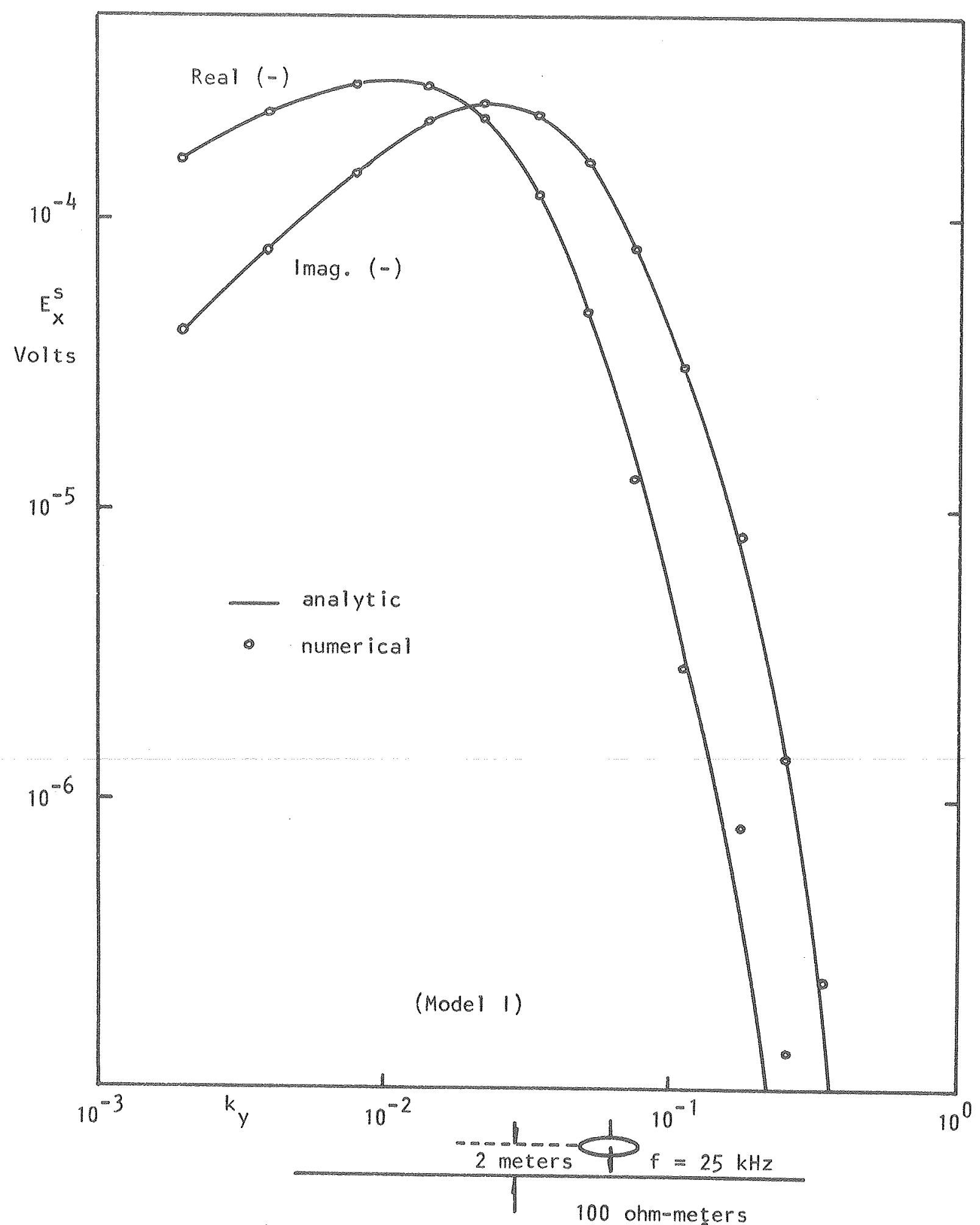

Figure 4. The secondary electric field $\left(E_{x}^{S}\right)$ in harmonic space on the surface 18 meters to the right of source 


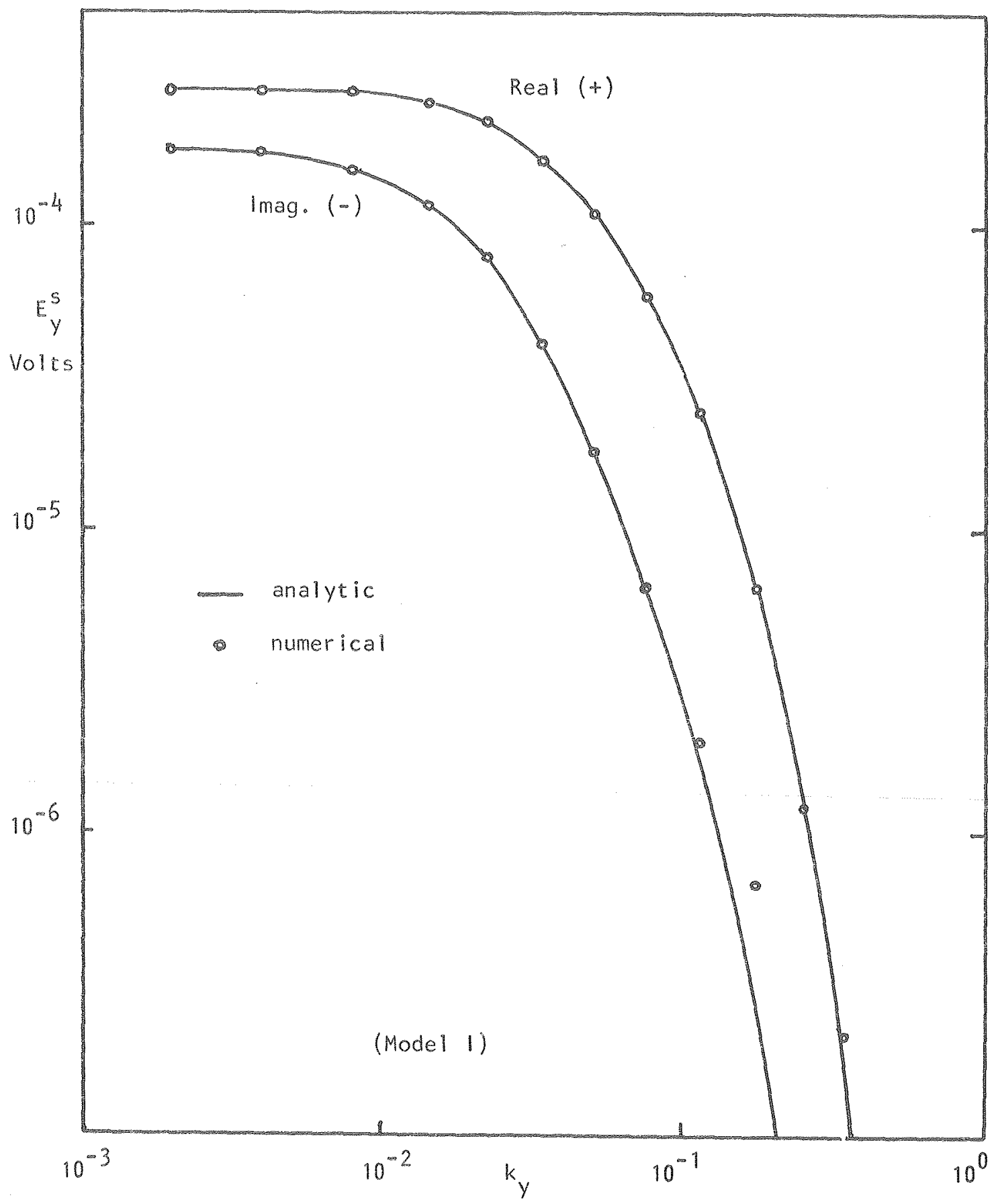

Figure 5. The secondary electric field $\left(E_{y}^{5}\right)$ in harmonic space on the surface 18 meters to the right of source

XBL $789-10949$ 


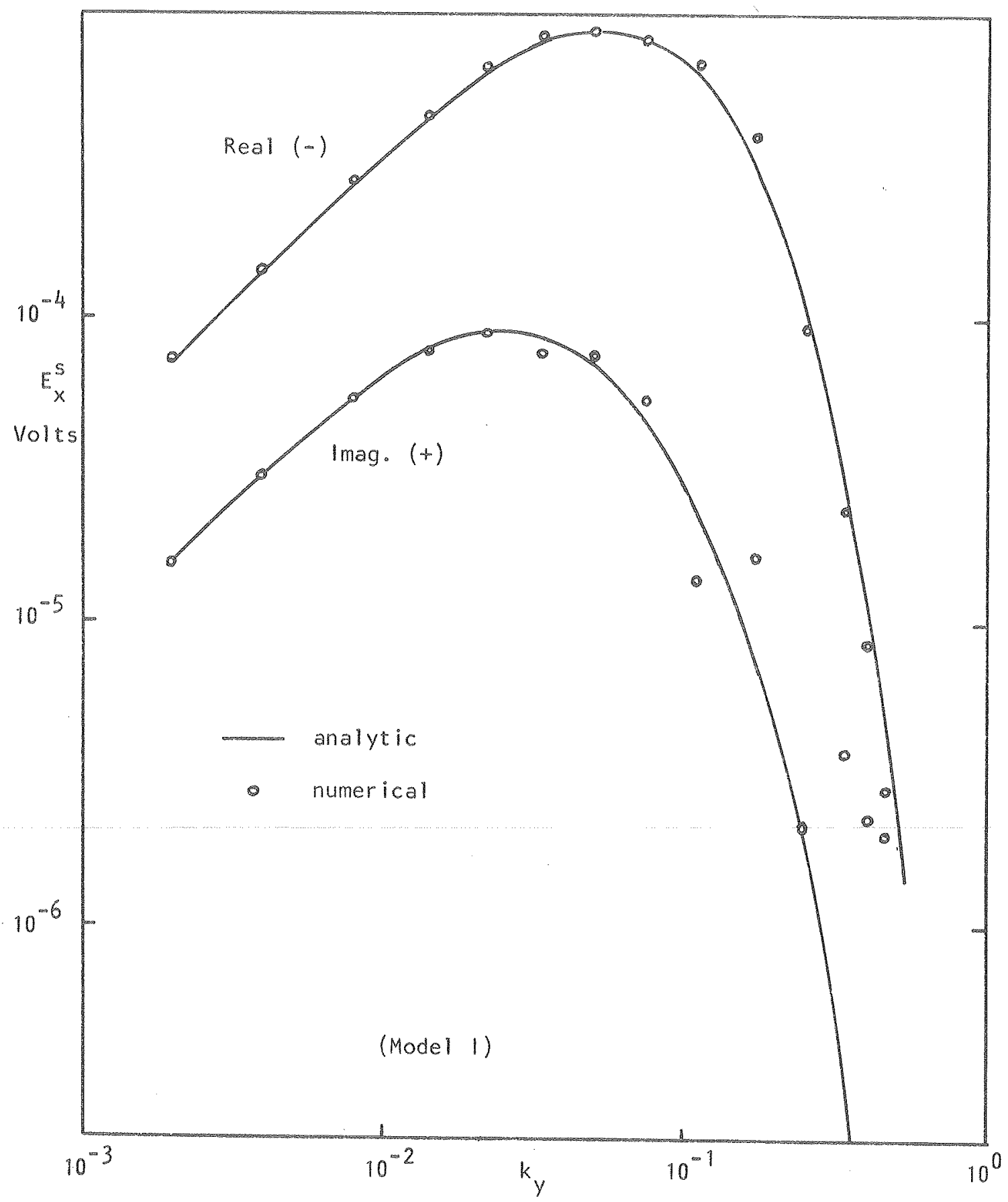

Figure 6. The secondary electric field $\left(E_{x}^{5}\right)$ in harmonic space on the surface 18 meters to the right of source

XBL 789-10950 


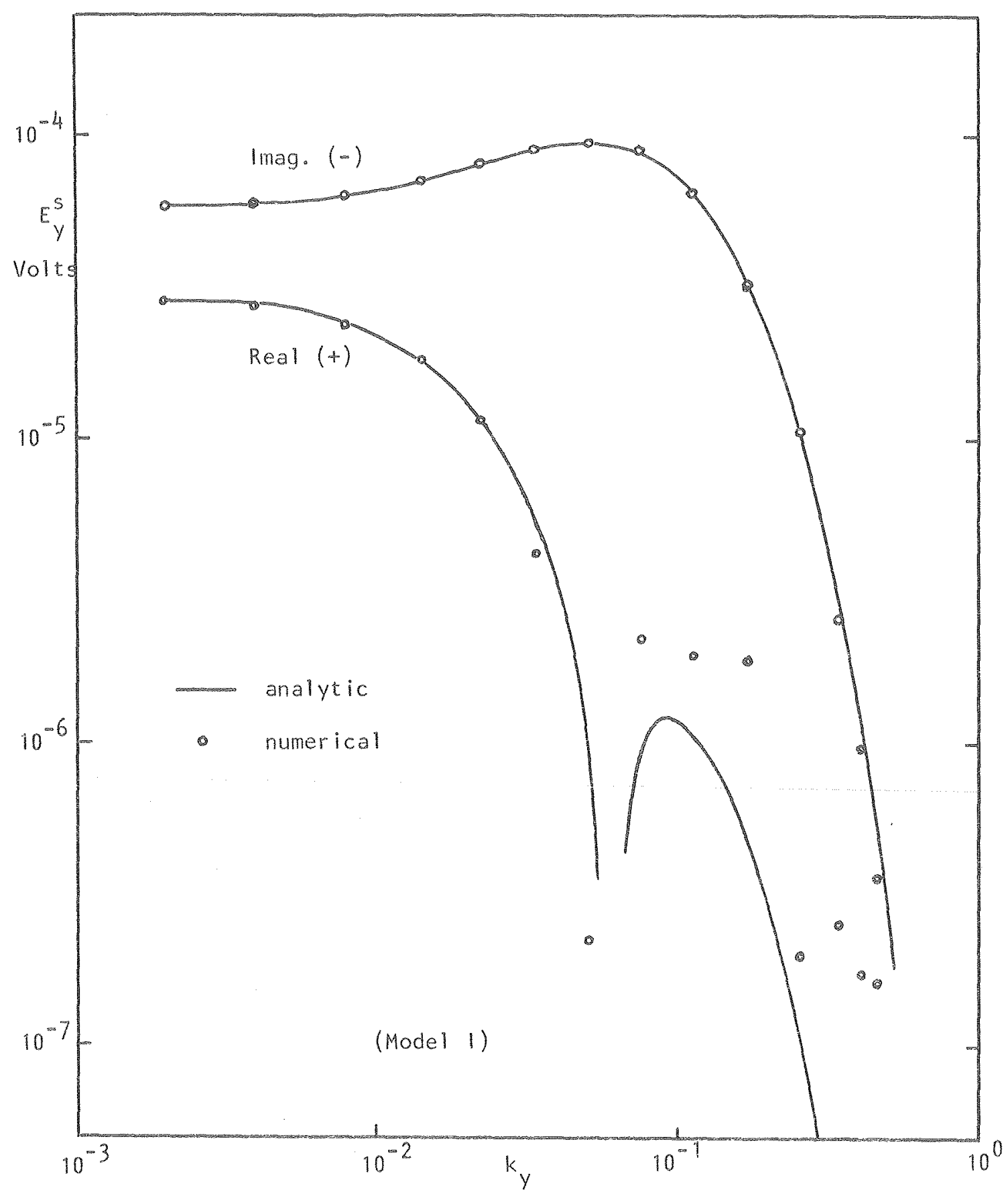

Figure 7. The secondary electric field $\left(E_{y}^{5}\right)$ in harmonic space on the surface 18 meters to the right of source

XBL $789-10951$ 
imaginary part of $E_{x}^{s}\left(k_{y}\right)$ and in the real part of $E_{y}^{s}\left(k_{y}\right)$ at higher values of $k_{y}$. These errors are considered unavoidable with this method since we have deliberately introduced the cause of these errors in the process of discretizing the variational integral. Although the concept of a soft boundary (appendix II) guarantees its continuity, the norma I component of electric fields changes so rapidly across the air-toearth interface that the common type of base functions described in a finite element may not be appropriate. Physically, ignoring displacement current, the vertical electric field completly shifts its phase across the interface due to the charges accumulated on the surface. Since the vertical electric field had to be forced to be continuous across the air-to-earth interface in the numerical regime, the error would also appear in the tangential components of electric fields through coupling as has been shown by the figures 6 and 7 .

Given the base function, the accuracy of the finite element solution is primarily dictated by the cell size. The cell size is usually described in terms of a fraction of a skin depth characterizing the degree of attenuation of the propagating field intensity. Since the effective skin depth gradually decreases and finally becomes inversely proportional to $k_{y}$ as $k_{y}$ increases, the cell size should also decrease accordingly. Redefining the cell size of a fixed model is time consuming, yet the scheme is necessary to retain the consistency of solution qualities for varying $k_{y}$ values. This has been done by dividing the desired $k_{y}$ range into a few bands, for each of which the proper cell size has been described.

The secondary magnetic fields have been numerically computed by Maxwe 17's equation (I-2). The necessary derivatives of electric fields 
have been obtained by initially interpolating them over the area of interest. Each component of the secondary electric fields has been interpolated by an optimum bi-cubic spline fit algorithm.

The secondary electric and magnetic fields in $y$ may be obtained by inverse Fourier transformation. The Fourier integrals given by (II-1) through (II-6) have been used for this purpose. The harmonic fields have been interpolated in wave number $k_{y}$. A three point quadratic interpolation scheme has been used for the cumulative sum of a piece wise Fourier integral

$$
f(x, y, z)=\frac{1}{\pi} \sum_{i=1}^{p} \int_{k_{y}\left(k_{i}\right)}^{k_{y}\left(u_{i}\right)} f\left(x, k_{y}, z\right)\left(\begin{array}{c}
\cos k_{y} y \\
j \sin k_{y} y
\end{array}\right) d k_{y},
$$

where $\quad U_{i}=2 i+1$ and $\ell_{i}=2 i-1$ with $\quad k_{y}(1)=0$. The number of piece wise integrations $p$ is typically 7 , or 15 wave numbers roughty logarithmically spaced. The zero harmonic fields for those components symmetric in $y$ may be easily deduced to be the same as the lowest harmonic components (refer to the Figures 5 and 7). For those components anti-symmetric in $y$ the zero harmonic fields are analytically zero.

Stoyer (1974) used this interpolation scheme in his development of a finite difference solution. He checked the quality of this interpolation scheme with an analytically calculated half space solution, and found that the error contained in the reproduced solution using 17 harmonics is within one percent.

\section{B. Numerical check}

The secondary electric fields in the space domain have been computed using equation $(I V-4)$. The harmonic electric fields were those previously calculated in the presence of a vertical magnetic dipole located 2 meters above a half space of 100 ohm-meter resistivity, Model I. 
Two horizontal components of the secondary electric fields have been plotted in Figure 8. The apparent radial symmetry of the horizontal electric fields can be observed. A maximum difference of 5 percent in magnitude can be seen between the two Cartesian components, $E_{y}$ and $-E_{x}$, computed along the two horizontal axes, $x$ and $y$, respectively. The results show one of the internal consistencies of the solution developed here.

Another test run has been made on the same model, Model I, to ensure the reciprocity between field components: $H_{x}$ due to a vertical magnetic dipole and $H_{2}$ due to a horizontal magnetic dipole. In either case the source is located at the surface of 100 ohm-meter half space and the frequency is $100 \mathrm{~Hz}$. The total fields have been obtained by superposing the primary and the secondary fields. The primary field in free space can be easily computed using equations (II-26) and (III-27), for which the primary magnetic Hertz potential $\pi^{*}$ is given by

$$
\bar{\pi}^{* P}(r)=\frac{\bar{M}_{3}}{4 \pi} \frac{e^{-j k_{0} r}}{r}
$$

The magnitudes and phases of $H_{x}$ and $H_{z}$ have been plotted in Figure 9 and Figure 10, respectively. In each Figure, curve 1 represents $H_{x}$ due to a vertical magnetic dipole, and curve 2 represents $H_{z}$ due to a horizontal magnetic dipole. Approximately a difference of two orders of magnitude has been observed between the two curves. One of the possible reasons for the vast discrepancy may have been that the source, in terms of the primary fields in this case, has not been treated properly in the process of discretizing the variational integral in section III. 


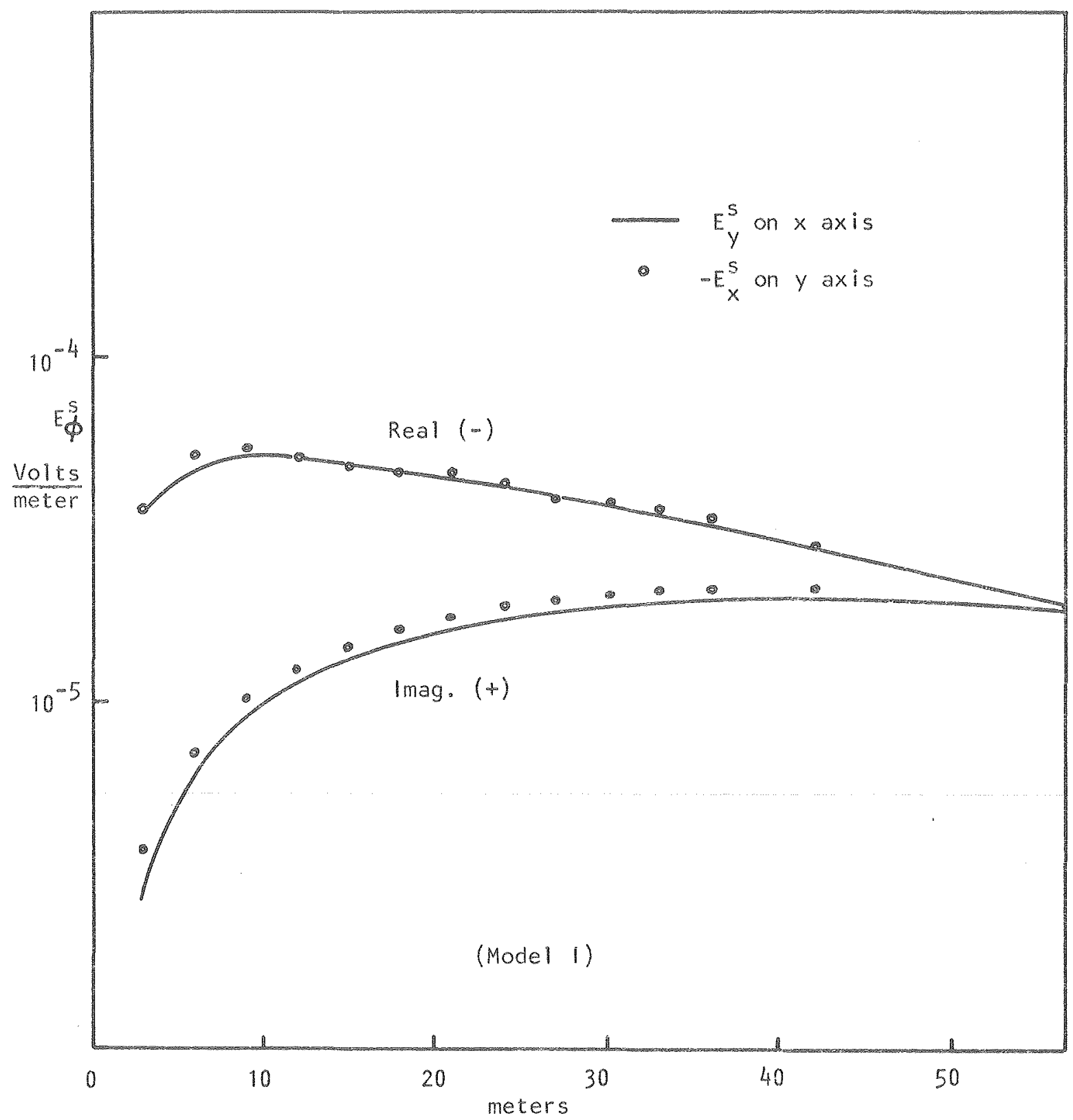

Figure 8. The symmetry of horizontal electric fields measured on the surface

XBL $789-10952$ 


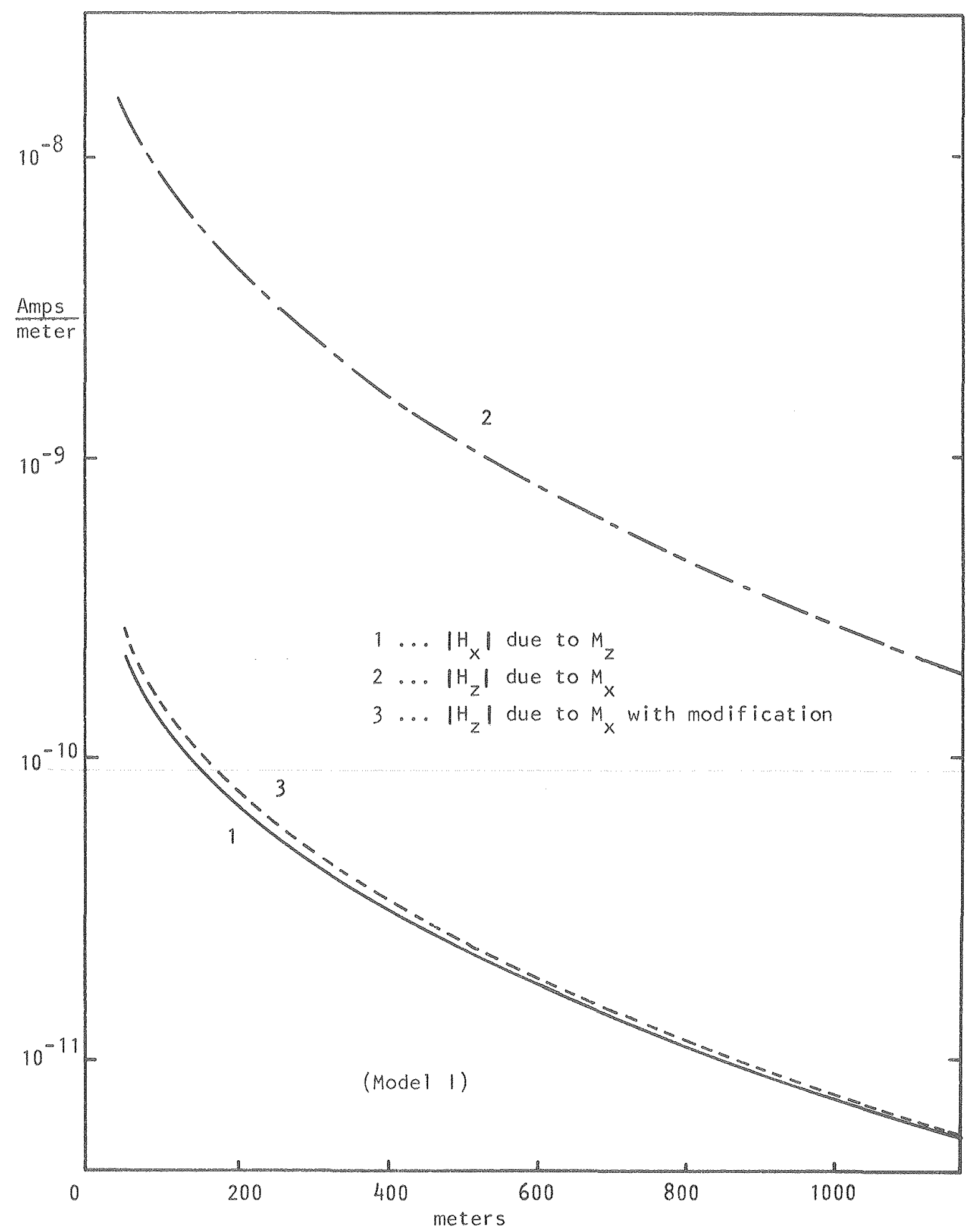

Figure 9. A reciprocity check between $\left|H_{x}\right|$ and $\left|H_{z}\right|$ 


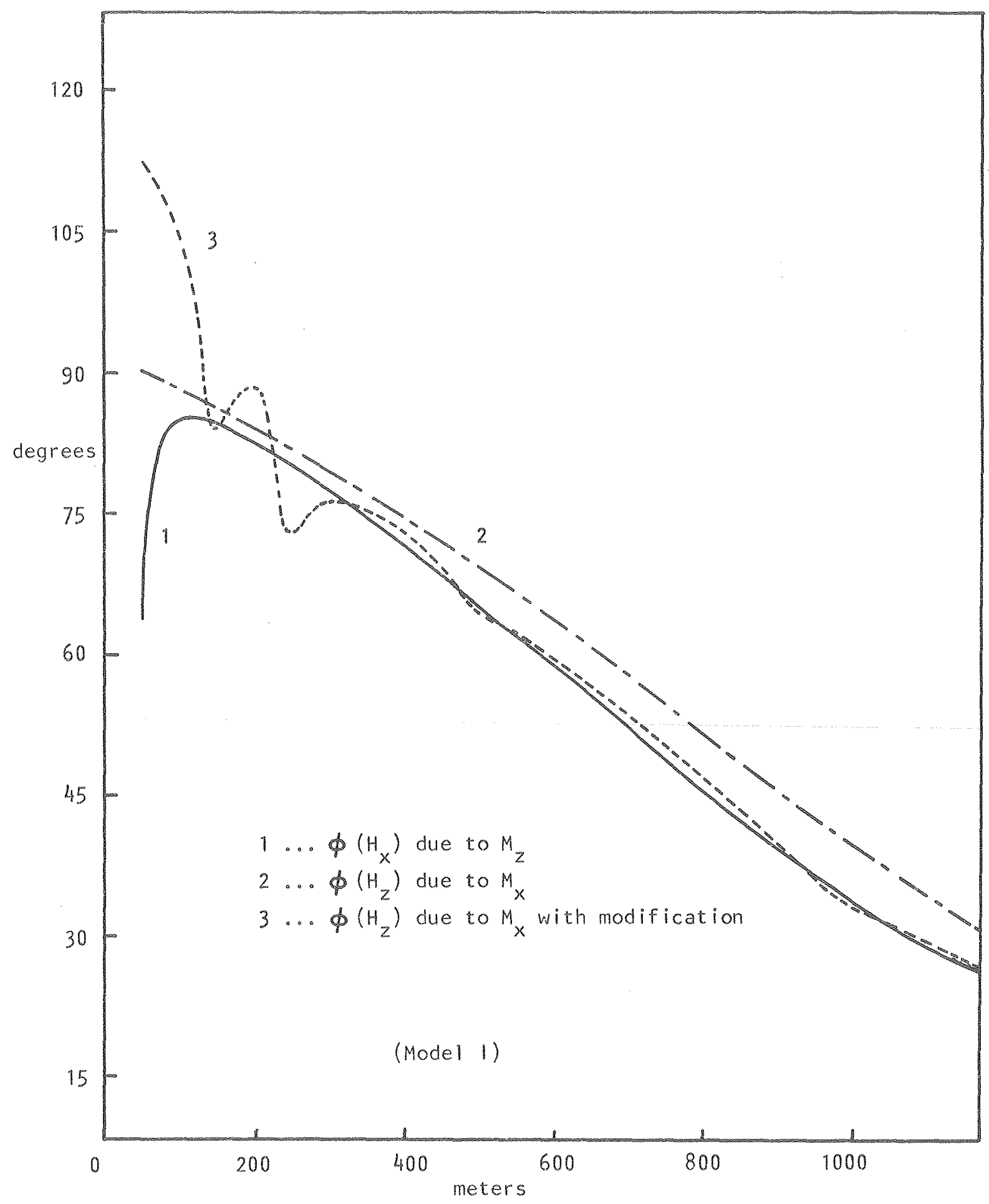

Figure 10. A reciprocity check between the phases of $H_{x}$ and $\mathrm{H}_{z}$ 
Rewriting the last term of the right hand side of equation (III-11) and designating it I yields:

$$
I_{s}=\frac{L}{L} \int_{s} \frac{k_{s}^{2}}{\omega^{2} \mu}\left(-E_{x}^{s} E_{x}^{p}+E_{y}^{s} E_{y}^{p}-E_{z}^{s} E_{z}^{p}\right) d x d z \text {. }
$$

within a very small rectangle in the immediate vicinity of source, Figure 11, the secondary electric fields may be treated as constants in contrast with the rapidly changing primary fields. The integration of the common non-zero term $E_{y}^{S} E_{y}^{P}$ can be written as

$$
I_{s}^{V}=-c \int_{x_{1}}^{x_{2}} \int_{z_{2}}^{z_{3}} \frac{\gamma\left(x-x_{1}\right)}{r} k_{1}(y r) d z d x \text {, }
$$

in the presence of a vertical magnetic dipole, and

$$
I_{S}^{H}=c \int_{x_{1}}^{x_{2}} \int_{z_{2}}^{z_{3}} \frac{\gamma(z+h)}{r} K_{1}(\gamma r) d z d x,
$$

in the presence of a horizontal magnetic dipole, where

$$
C=\frac{\sigma \mu}{2 \pi L} E_{y}^{s}
$$

and

$$
r=\left\{\left(x-x_{1}\right)^{2}+(3+h)^{2}\right\} 1 / 2
$$

Changing variables, $x-x_{1} \rightarrow x$ and $z+h \rightarrow z$, and taking the leading term $1 / y r$ of the series representation of $k_{j}(y r)$, which is valid for small yr (Abramowitz and Stegun, 1964), we can approximately evaluate the integral (IV-7) as

$$
\begin{aligned}
I_{S}^{V} & =-C \int_{h}^{h+D z} \int_{0}^{D x} \frac{x}{x^{2}+z^{2}} d x d z \\
& =-\frac{C}{z} \int_{h}^{h+D z} \ln \left(\frac{D x^{2}+z^{2}}{z^{2}}\right) d z .
\end{aligned}
$$

If we let $D X=D Z$ and $t=D X / z$, then the last integral for $h \rightarrow 0$ becomes (Gröbner and Hofreiter, 1958) 


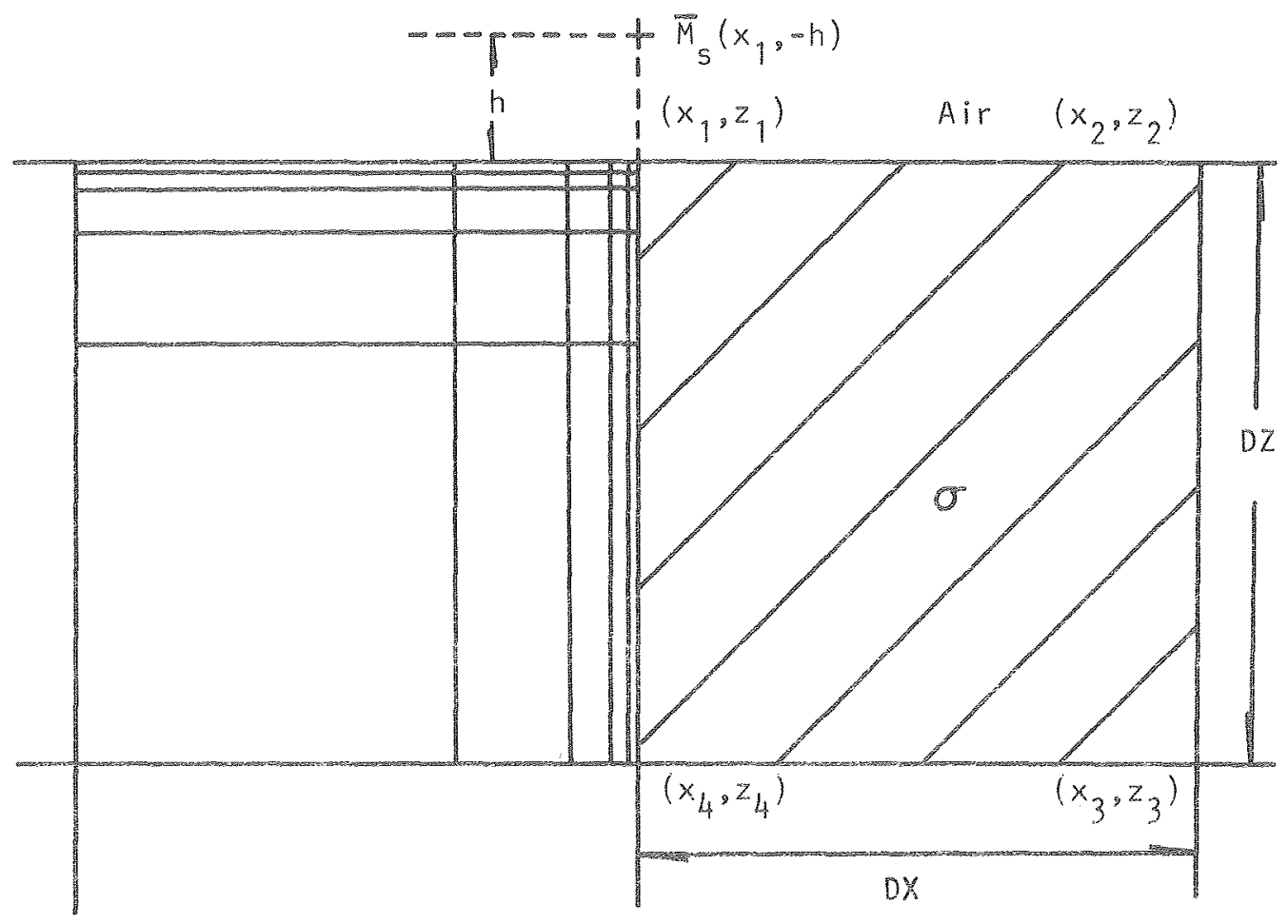

Figure 11. Finite elements in the immediate vicinity of source

XBL $789-10955$ 


$$
\begin{aligned}
\lim _{h \rightarrow 0} I_{s}^{V} & =-\frac{c}{2} D x \int_{1}^{\infty} \ln \left(1+t^{2}\right) \frac{d t}{t^{2}} \\
& =-c\left(\frac{\pi}{4}+\frac{\ln 2}{2}\right) D x .
\end{aligned}
$$

Similarly, in the presence of a horizontal magnetic dipole, equation (IV-8) can be approximated as

$$
I_{5}^{H} \doteq \frac{C}{2} \int_{0}^{D x} \ln \left(\frac{x^{2}+(D z+h)^{2}}{x^{2}+h^{2}}\right) d x .
$$

Changing variable $x=h t$, one can show that (Grobner and Hofreiter, 1958)

$$
\lim _{h \rightarrow 0} I_{S}^{H}=C \frac{\pi}{2} D X
$$

Keeping in mind those pseudo-analytic results given by equations (IV-10) and (IV-12), let us find out what source term we had for the numerical equation (II-12). Assuming constant secondary electric fields and using the same approximation for $k_{1}(\gamma r)$, we may write the source term due to a vertical magnetic dipole as

$$
\begin{aligned}
I_{s}^{V} & =-c \int_{0}^{D z} \int_{0}^{D x} \sum_{i=1}^{L} N_{i}\left\{\frac{x_{i}}{x_{i}^{2}+(3 i+h)^{2}}\right\} d x d z \\
& =-c \frac{D x \cdot D Z}{4}\left\{\frac{D x}{D x^{2}+h^{2}}+\frac{D x}{D x^{2}+(D E+h)^{2}}\right\} .
\end{aligned}
$$

In the presence of a horizontal magnetic dipole, we have

$$
\begin{aligned}
I_{-5}^{H} & \doteq C \int_{0}^{D E} \int_{D}^{D x} \sum_{i=1}^{4} N_{i}\left\{\frac{z_{i}+h}{x_{i}^{2}+\left(z_{i}+h\right)^{2}}\right\} d x d z \\
& =C \frac{D x \cdot D z}{4}\left\{\frac{1}{h}+\frac{1}{D x^{2}+h^{2}}+\frac{D z+h}{D x^{2}+(D z+h)^{2}}+\frac{1}{D z+h}\right\} .
\end{aligned}
$$

Hence, with $D X=D Z$, we obtain 


$$
\begin{aligned}
& \lim _{h \rightarrow 0} I_{s}^{V} \doteq-c \frac{3}{8} D x, \\
& \lim _{h \rightarrow 0} I_{s}^{H} \doteq c \frac{D x^{2}}{4} \lim _{h \rightarrow 0} \frac{1}{h} .
\end{aligned}
$$

When the source is close to or on the surface, the fcregoing analyses suggest us the following conclusions:

(1) the numerically computed source term in the presence of a vertical magnetic dipole is independent of source height $h$ and in fact is similar to the one analytically computed.

(2) In view of conciusion (1), the numerically obtained vertical magnetic dipole solution will be effectively the same as the analytic solution.

(3) unless the cell size DX is comparable with source height $h$, the solution obtained in the presence of a horozontal magnetic dipole wi11 be absurd.

In compliance with conclusion (3), the modified source term has been computed initially by subdividing the cell in the immediate vicinity of source (refer to the cell on the left in Figure 11). Assuming that the primary field is bi-linear in each one of the subdivided cells, the numerical representation of the each source term in equation (III-11) can be rewritten as

$$
E^{s T} \sum_{i=1}^{p} \frac{1}{L} \int_{\Delta_{i}} \frac{k_{s}^{2}}{\omega^{2} \mu} N \underline{L}^{i} T \stackrel{i}{E}^{i} d x d z \text {. }
$$

where $p$ is the number of subdivided cells and $\underline{N}^{L^{2}} \underline{E}^{p}$ is the primary electric field in the $i^{\text {th }}$ subdivided cell. The secondary electric field remains bi-linear in the original cell. 
Curve 3 drawn in Figure 10 and Figure 11 has been obtained respectively in the presence of a horizontal magnetic dipole using the result given by (IV-17). In the vicinity of the source a maximum of 15 percent difference in magnitude can be observed. However, the phase of $\mathrm{H}_{\mathrm{z}}$ shows substantial fluctuations mostly confined in the region within a half skin depth from the source. The vertical electric field across the air-to-earth interface seems to have played a major role in causing the fluctuation. Although the solution shows near source phase fluctuations in $\mathrm{H}_{z}$, we consider that reciprocity apparently holds.

The solution with the modified source term given by $(I V-17)$ due to a vertical magnetic dipole has not been plotted because it almost overlaps the original solution, curve 1 , as has been predicted by conclusion (2). The fact that the two solutions are identical also indicates that the solution is convergent.

The validity of the solution presented here has been tested on a two-layered half space, Model II, consists of a 50 meters thick overburden of 10 ohm-meter resistivity over a 100 ohm-meter half space. A vertical magnetic dipole of unit moment is located at . 1 meter above the surface and 100 meters to the left of the observation point. Figure 12 and Figure 13 show the analytically computed frequency sounding curves in terms of magnitudes and phases of $H_{x}$ and $H_{z}$, respectively. Three frequencies, $36 \mathrm{~Hz}, 2.5 \mathrm{kHz}$, and $10.0 \mathrm{kHz}$ have been selected on the basis of their significant roles in the curves. The numerical solutions for the selected frequencies are almost identical to the analytic solutions except for the phase of $\mathrm{H}_{z}$ at $10.0 \mathrm{kHz}$, where the phase is analytical1y minimim.

As an absolute check regarding the quality of the solution, it 


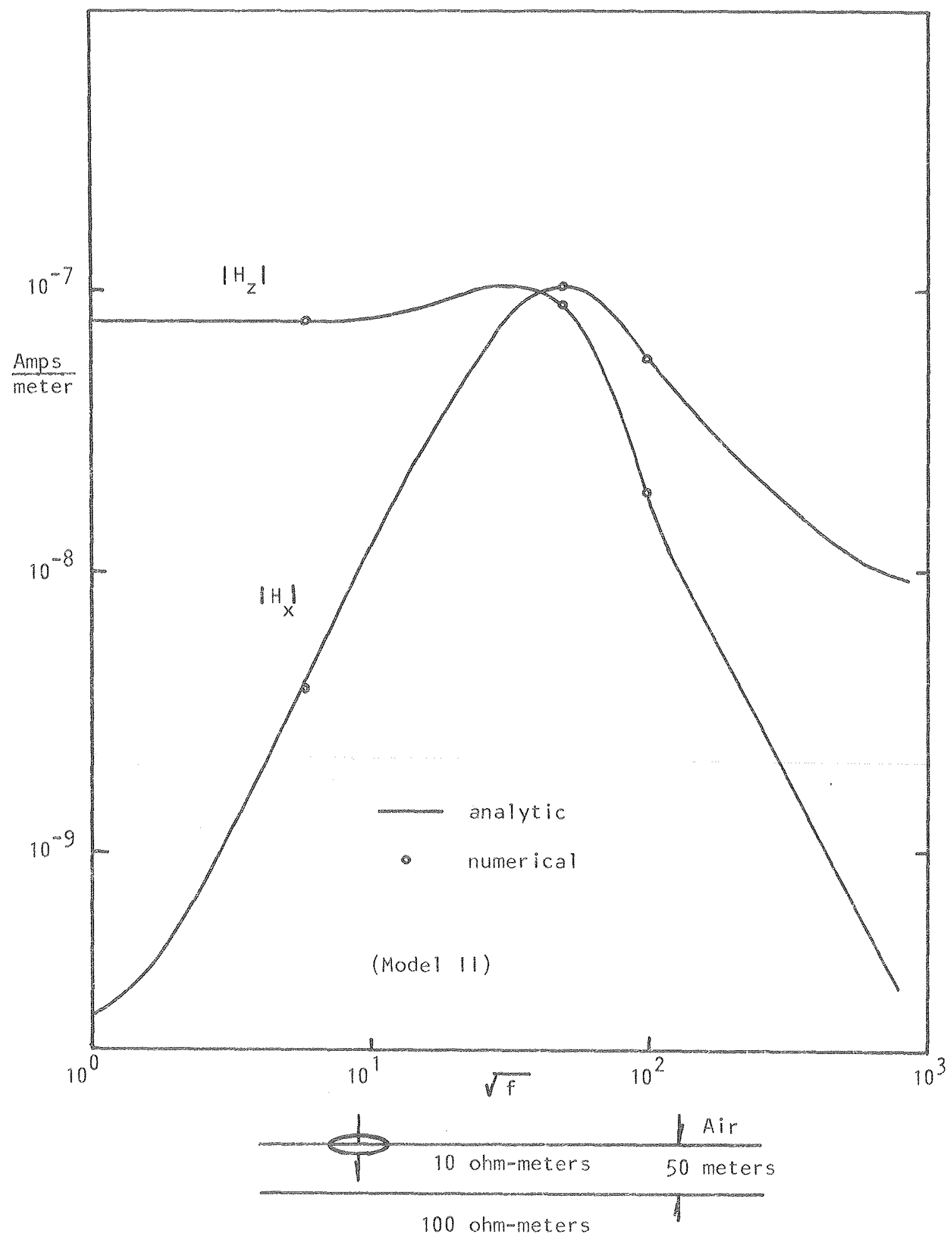

Figure 12. The frequency sounding curves in magnitudes on a two-layered half space

XBL 789-10967 


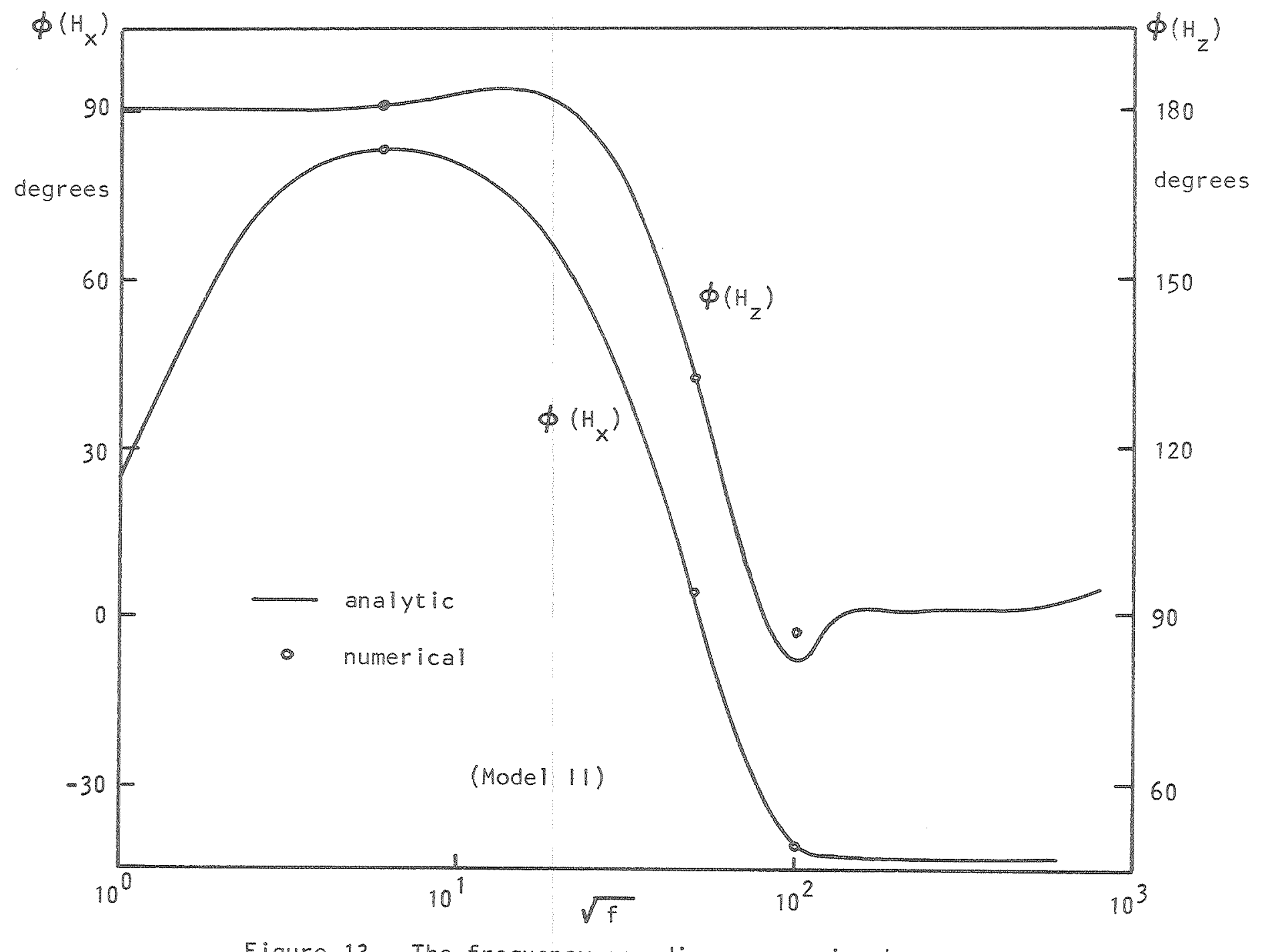

Figure 13. The frequency sounding curves in phases on a two-layered half space

XBL $789-10956$ 
would be preferable to have another two-dimensional solution developed independently from the method described here. Unfortunately, due to the lack of a competitive solution, we have not been able to make a direct comparison between the two-dimensional solutions. As a matter of convenience, three-dimensional model results have been used for the numerical checks.

One of the solutions for three-dimensional electromagnetic scattering problems has been developed by Pridmore (1978) using finite element technique. The scattering by a 1 ohm-meter conductor of size $30 \times 120 \times 90$ cubic meters buried in the half space of 30 ohm-meter resistivity has been plotted in Figure 14 and Figure 15 in terms of the real and the imaginary parts of the secondary $\mathrm{H}_{z}$, respectively. The depth of burial is 30 meters and the source is a vertical magnetic dipole located at .1 meter above the surface 75 meters to the left of the center of the conductor. The given magnetic moment is $4 \pi$ ampere-meter $^{2}$ and the frequency used is $1.0 \mathrm{kHz}$. The anomaly in $\mathrm{H}_{2}$ plotted in the Figures has been obtained in the following two steps (Pridmore, 1978); 1) the electric field in the conductor has been calculated by the finite element method, 2) the magnetic field at the surface has been obtained by integrating the inner product of the half space Green's tensor for $\mathrm{H}_{2}$ and the scattering current, the total electric field multiplied by the excessive conductivity of the inhomogeneity.

The numerical solutions obtained for the two-dimensional conductor with the same cross sectional geonetry, Model III, have also been plotted in the same Figures. Since the solutions we have generated are the total half space responses including the scattered fields by the conductor, the plotted quantities have been obtained by subtracting the analytically 



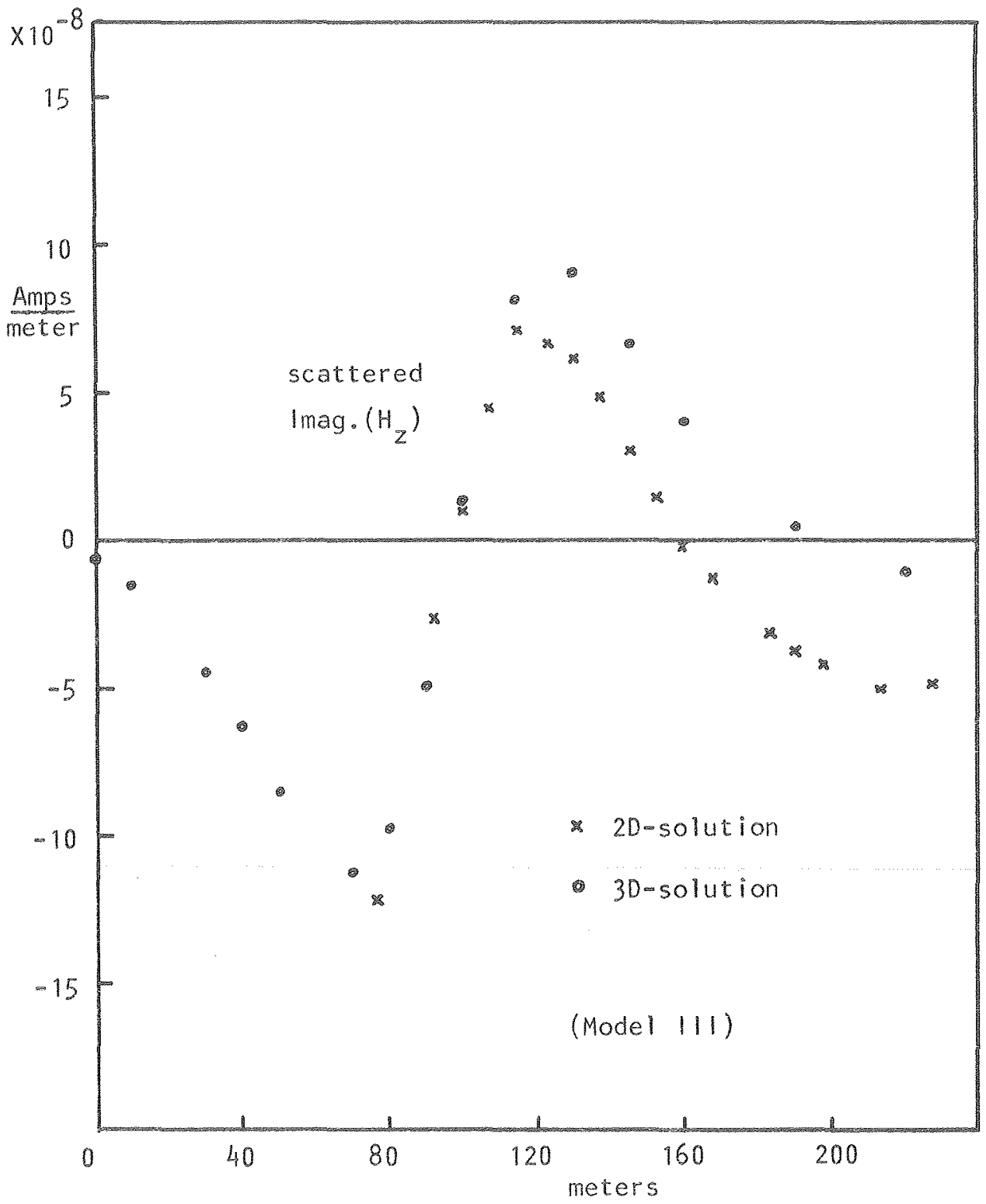

Figure 15. The comparison between $2 D$ and $3 D$ solution. The strike length of 30 body is 120 meters 
computed half space responses from the solutions originally obtained. The solutions in the vicinity of source have not been plotted simply because we know that the contributions from the range beyond the cutoff $k_{y}$ are too significant to be truncated. It has been found that the convergence of harmonic solutions in $k_{y}$ is very slow in the vicinity of source, especially for the imaginary part of $\mathrm{H}_{z}$. In the regions of convergence however, the two solutions share the same zero crossings on top of the conductor, both for the real and the imaginary parts of the anomalous $\mathrm{H}_{2}$. After the zero crossings the two anomalies differ considerably in their assymptotic behaviour at increasing distance from the conductors. As can be intuitively expected, the anomaly due to a twodimensional conductor diminishes much slower than one due to a threedimensionat conductor.

Another numerical check has been made by comparing measurements made on one of Frischknecht's tank models and the solution obtained here for a two-dimensional body. In this model a transmitter-receiver pair with a separation of 2000 meters is moved on the surface across the center of the conductor. The scaled two-dimensional numerical model, Model IV, consists of a half space of 13.7 ohm-meter resistivity, in which a 1.82 ohm-meter conductor of size $500 \times 500$ square meters is buried. The scaled length of the tank model is 3000 meters in the strike direction. The depth from the surface to the top of the conductor is 200 meters. The frequency used is $.15 \mathrm{~Hz}$. In the presence of a vertical magnetic dipole, the normalized vertical magnetic fields, $H_{z} / H_{z}^{p}$, measured by the receiver versus the array center have been plotted in Figure 16. Except for the imaginary part in the region above the conductor, the two-dimensional body has a response of greater magnitude than the three- 


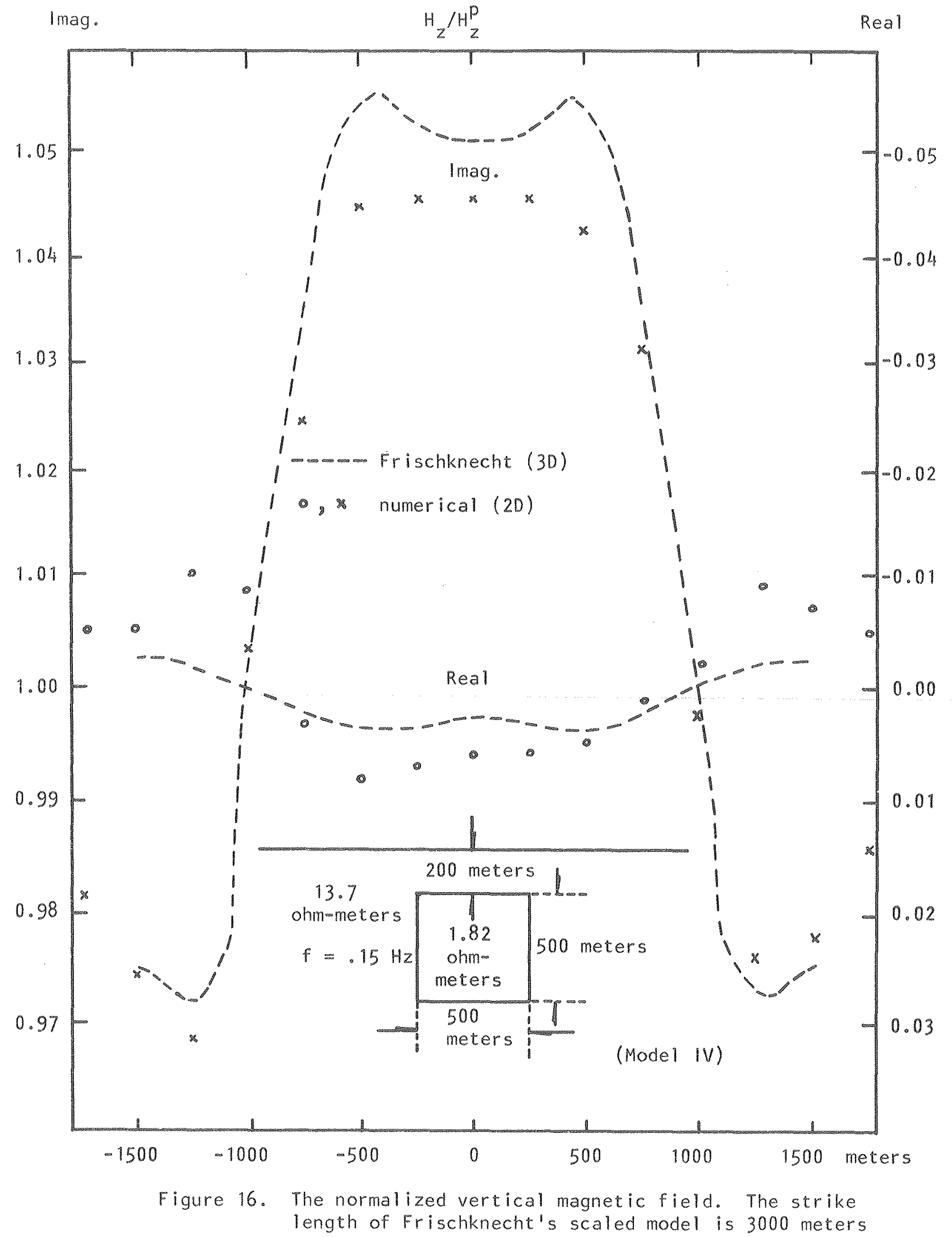


dimensional one. The asymmetry in the numerical solution across the center of the conductor is less than 1 percent with respect to the total field. Considering the limitations inherently carried in the numerical solution, the quality of the solution may well be accepta ble as far as the reciprocity principle is concerned. An additional reciprocity check has been made between $H_{x}$, Figure 17 , due to a vertical magnetic dipole and $H_{z}$, Figure 18, due to a horizontal magnetic dipole on the same model. The vertical magnetic fields plotted in Figure 18 are the values at 2000 meters to the left of the transmitting horizontal magnetic dipole. Again, we have reasonably good reciprocity check between the field components.

The next model shown here is a half space composed of two quarter spaces, Model $\mathrm{V}$; on the left of the contact the resistivity is $100 \mathrm{ohm}$ meters, and on the right, 10 ohm-meters. The array consists of a vertical magnetic dipole of unit moment and a receiver 200 meters to the right, moved along the earth surface in the direction perpendicular to the contact. The frequency used is $100 \mathrm{~Hz}$. The magnitudes and the phases of $E_{y}, H_{x}$, and $H_{z}$ versus array centers have been plotted in Figure 19, Figure 20, and Figure 27, respectively. On the resistive side far away from the contact, the current flows circularly with its phase lagging approximately 90 degrees behind the transmitting magnetic moment. As the array approaches to the contact from the resistive side, the conductive quarter space starts drawing source current and thus the field decreases. This channeling of the current in the conductive region across the contact reaches its maximum as the array straddles the contact. The horizontal magnetic field $H_{x}$ increases as expected an order of magnitude from one side to the other. Approximately 25 degrees 


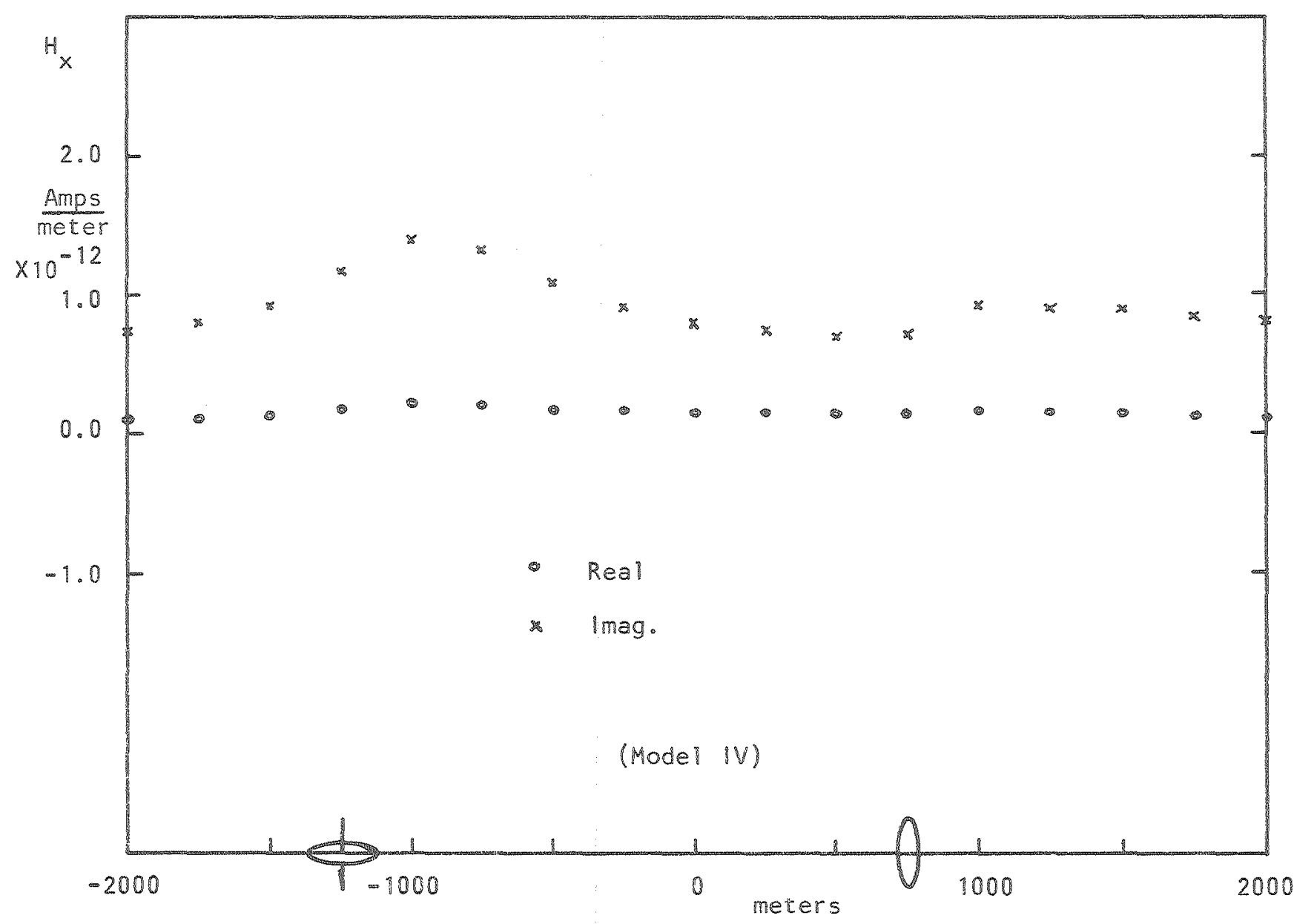

Figure 17. The horizontal magnetic field, Hx, due to a vertical magnetic dipole 


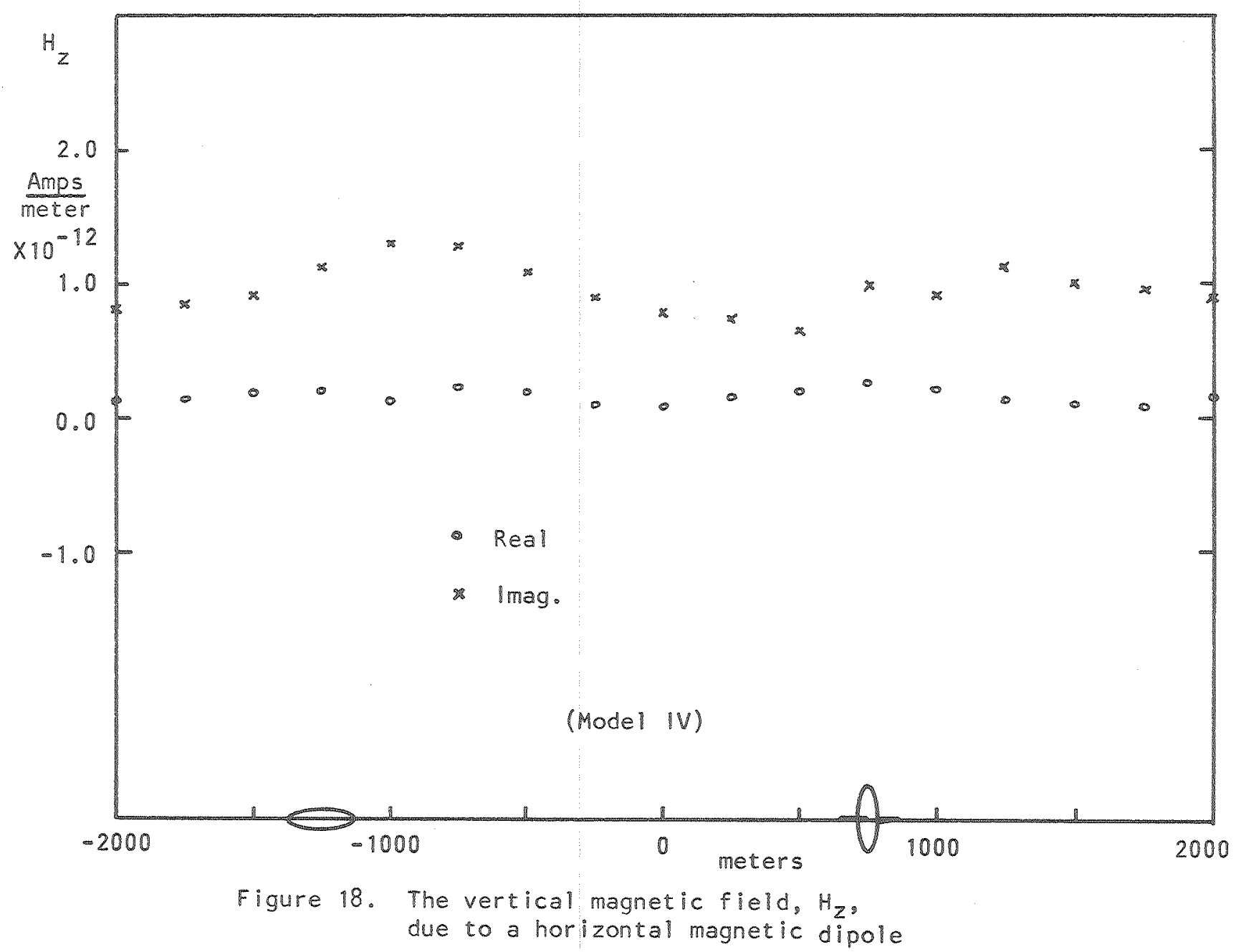

XBL 789-10961 


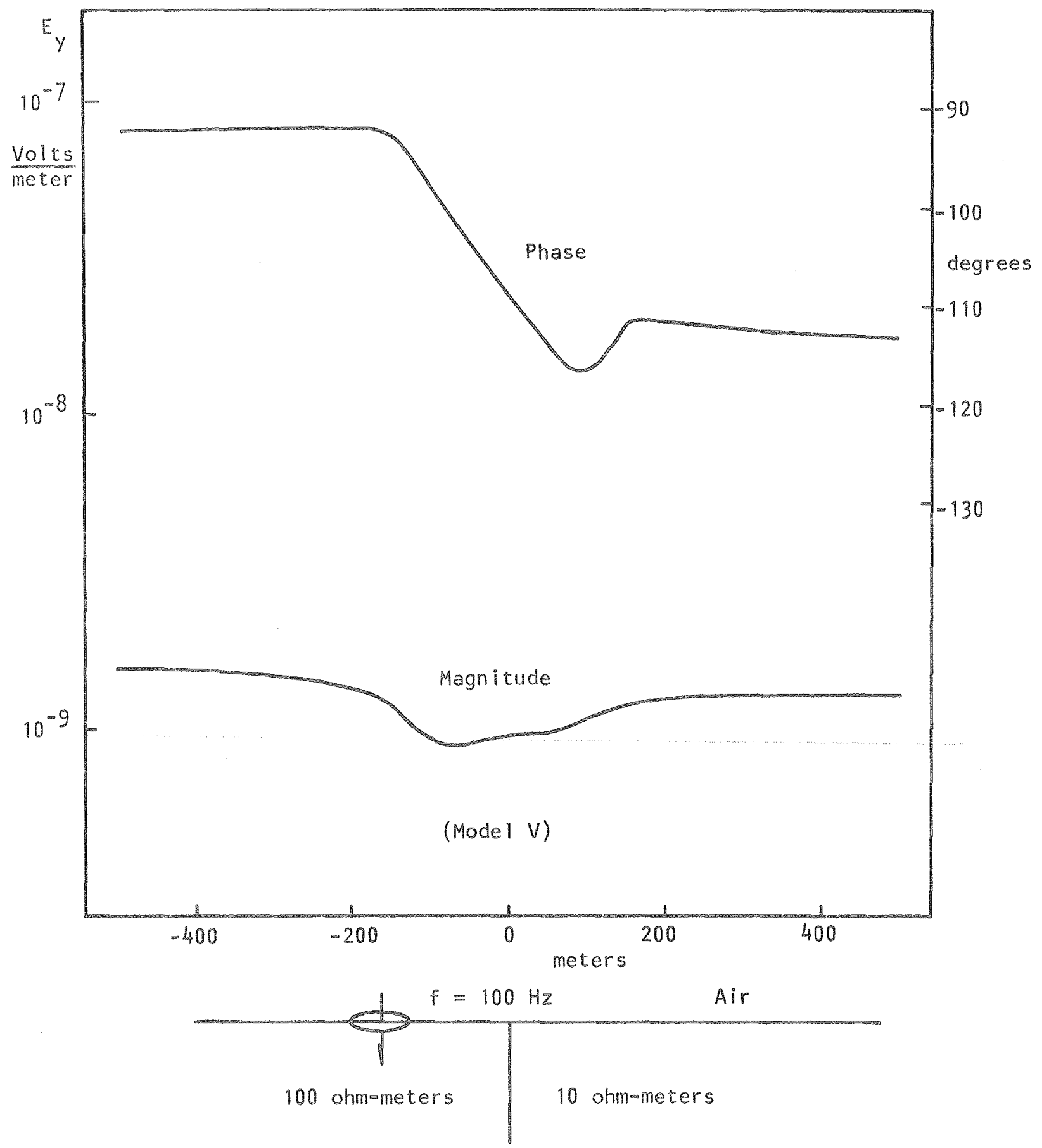

Figure 19. Contact response in $E_{y}$

XBL $789-10962$ 


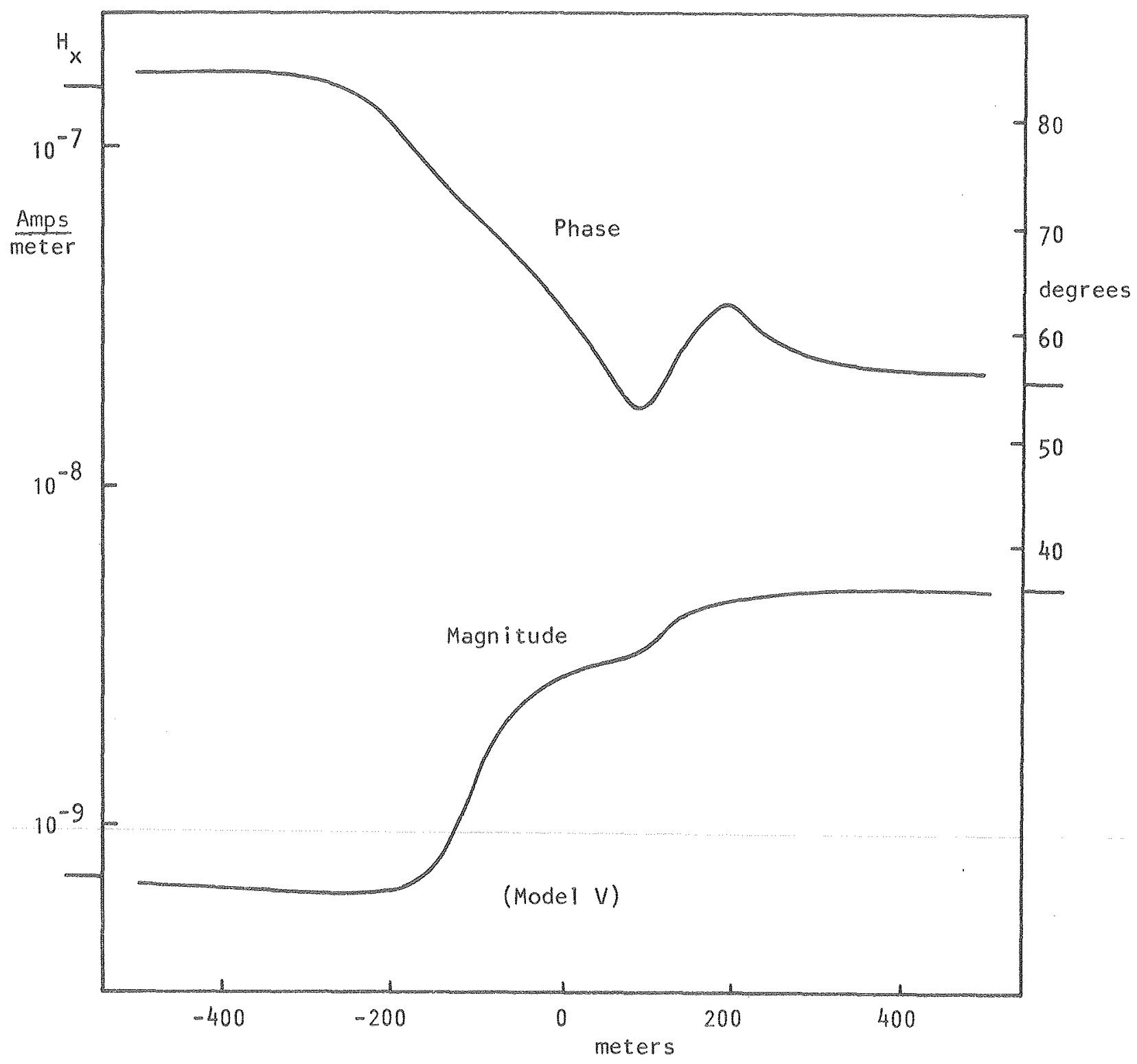

Figure 20. Contact response in $H_{x}$

XBL 789-10963 


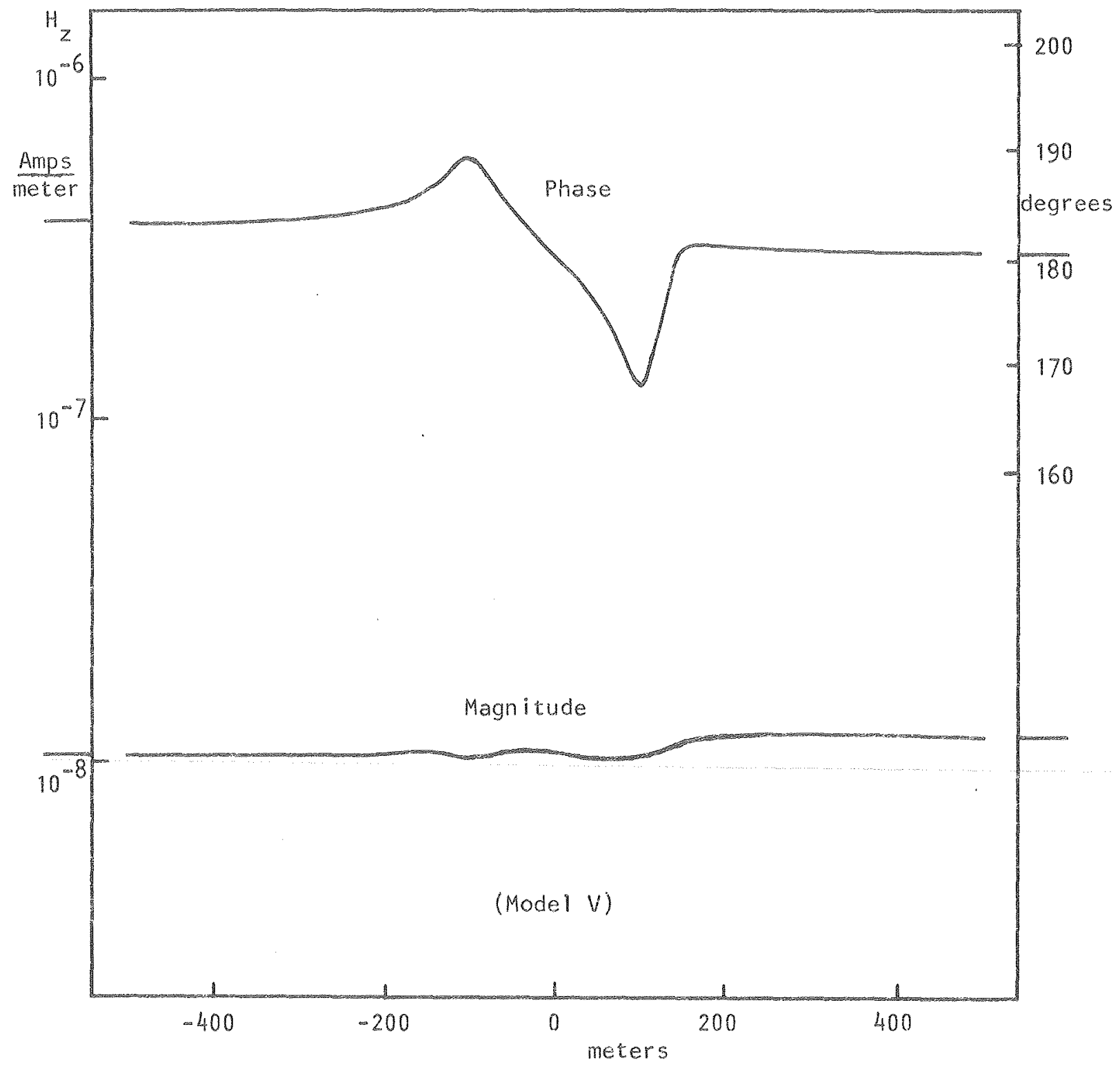

Figure 21. Contact response in $H_{z}$

XBL 789-10964 
of phase retardations can be observed for all the field components as increasingly more waves propagate through conductive medium with their phases lagging behind. Since the real part of $\mathrm{H}_{z}$ is strongly dominated by the primary dipolar field, the observed phase change in $\mathrm{H}_{2}$ across the contact indicates a rapid variation in the imaginary part of $\mathrm{H}_{\mathrm{Z}}$. The relative lateral position of the channeling current with respect to the array configuration is primarily responsible for the shape of the observed variations in each component.

For all the field components there is little effect of the contact once the transmitter or receiver is 200 meters from it. With increasing transmitter-receiver separation, it is anticipated that the shape of anomaly for each component will become broader and smaller. The long tick marks on both ordinates of $H_{x}$ and $H_{z}$ are half space analytic solutions to which the numerical solutions converge. 
V. Conclusion

A finite element method has been developed for determining the electromagnetic scattering by an arbitrary two-dimensional inhomogeneity in the presence of a magnetic dipole. Although we have considered only a magnetic dipole, the program developed herein can be used for any finite source, provided that the corresponding one-dimensional analytic solution is known.

The quality of the solution for a horizontal magnetic dipole has been shown to be relatively poor compared to that of the vertical magnetic dipole. The difficulty stems primarily from the effect of charges accumulated at the air-to-earth interface. We have used a soft or gradational boundary as a substitute for the step like conductivity discontinuity, expecting that the distribution of charges would be continuous. At the air-to-earth interface however, the conductivity contrast is so severe that the normal component of electric fields is completely polarized in the opposite direction across the interface due to the surface charges. Consequently, there appears to be a physical limit beyond which a discretized numerical scheme, such as the one develeoped here, can not cope.

Another important factor concerning the quality of the solution, especially for a horizontal magnetic dipole, is the degree of accuracy of the calculated source vector including the boundary vaiues. It has been shown that a remarkable improvement in the solution quality can be achieved by using accurately evaluated source vector due to the primary field (refer to the brief analysis and conclusions made in section $B$, part IV). The fact that we can evaluate the source term accurately and use it in the finite element method is one of the major advantages 
over the finite difference method, such as the one developed by Stoyer, (1974). The trouble with the finite difference solution is that, without having an accurate source term implemented in the system equation, the phase of the calculated field or the ratio of one component to the other contained in the solution could be reasonably good regardless of the quality of the actual magnitudes of the calculated solution. Therefore, the validity of the solution must be verified by checking on the calculated field quantity itself.

The internal consistency of the algorithm has been demonstrated on the uniform half space model both for the vertical and the horizontal magnetic dipole solution. Numerical checks have been made against the solutions obtained for three-dimensional models. Except for the differences in shape, which we believe are due to the dimensionality of the conductive body used, it has been found that the solutions are basically similar. The two-dimensional anomaly, however, generally shows a slower assymptotic behavior with larger magnitude than the threedimensional one. The qualitative interpretations of the electromagnetic responses of a certain class of three-dimensional models, at least those illustrated for the comparisons in this thesis, may effectively be accomplished using much inexpensive two-dimensional model results.

For the inverse fourier transformation, we have used 15 harmonic solutions for all the models studied here. However, we may need more harmonic solutions if: 1) the model under study is such that the field is expected to behave rather rapidly in the direction parallel to the strike, a situation, for example, encountered by a model which has a surface inhomogeneity of high conductivity contrast, and 2) an accurate solution is desired in the vicinity of source. 
The primary purpose of the study presented here has been to investigate the electromagnetic responses of common geologic structures with inhomogeneities of large scale, such as contacts, semi-infinite surface or buried layers, and infinite dykes. The typical applications of the method are the model study of the electromagnetic sounding at the edge of a valley or the study of the electromagnetic coupling effect due to a grounded electric dipole necessary for the interpretation of IP: field data.

In the future, provided that the numerical as well as the physical difficulty of the air-to-earth interface is solved, the direct magnetic field solution will not only eliminate the unnecessary modifications made for the electric field solution, but undoubtedly improve the quality of the solution. 


\section{APPENDIX I}

Reduction of Variation Integral to Differential Equation

a) Magnetic Type

Rewriting equation (I-8)

$$
I(\bar{H})=\int_{v}\left[\frac{\mu}{2} \bar{H}^{2}-\frac{\mu}{2 k^{2}}(\nabla \times \bar{H})^{2}+\mu \bar{H} \cdot \bar{M}_{s}\right] d v \text {, }
$$

and using the vector identity

$$
\nabla \cdot(\bar{A} \times \bar{B})=\bar{B} \cdot \nabla \times \bar{A}-\bar{A} \cdot \nabla \times \bar{B} \text {, }
$$

the variation of $I(\bar{H})$ with respect to $\tilde{H}$ becomes

$$
\begin{aligned}
\delta I(\bar{H}) & =\int_{V} \mu\left(\bar{H}-\frac{1}{k^{2}} \nabla \times \nabla \times \bar{H}+\bar{M}\right) \cdot \delta \bar{H} d v \\
& +\int_{V} \frac{\mu}{k^{2}} \nabla \cdot(\nabla \times H \times \delta \bar{H}) d v \\
& \equiv 0 .
\end{aligned}
$$

By the divergence theorem and Maxwell's equations, the second integral in (3) can be written as

$$
-\int_{S} \frac{1}{j \omega} \vec{E} \times \bar{s} \cdot \vec{H} \cdot d a \text {, }
$$

where $\bar{n}$ is a unit vector outward normal to the surface $S$.

The surface integral (4) would vanish at every internal boundary because $\bar{n}$ alternates its sign between adjacent cells. The only nonzero contribution comes from the surface integral along the external boundary. We force this non-zero term to vanish by employing one of the following boundary conditions:

(1) tangential magnetic fields are prescribed on the surface, so that

$$
\bar{n} \times\left.\bar{H}\right|_{s}=\text { const. } \rightarrow \quad \bar{n} \times\left.\delta \bar{H}\right|_{s}=0 .
$$


(2) tangential electric fields are set equal to zero

$$
\bar{n} \times\left.\bar{E}\right|_{S}=0 .
$$

With either one of the boundary conditions satisfied, the volume integral must vanish. But since $\delta \bar{H}$ is arbitrary in the volume the coefficient has to be zero everywhere. Hence

$$
\bar{H}-\frac{1}{k^{2}} \nabla \times \nabla \times \bar{H}=-\bar{M}_{s} \text {. }
$$

\section{b) Electric type}

The variational integral in this case is

$$
\left.I(E) \times \int \sqrt{v} \frac{k^{2}}{2 \omega^{2} \mu} E^{2}-\frac{1}{2 \omega^{2} \mu}(\nabla \times E)^{2}+\frac{1}{j \omega} \cdot \bar{J}_{s}\right] d v \text {. }
$$

Taking variations of $I(\bar{E})$ with respect to $\bar{E}$ and following the procedure similar to the magnetic type case, we obtain

$$
\begin{aligned}
\delta I(\bar{E}) & =\int_{V} \frac{1}{\omega^{2} \mu}\left(k^{2} \vec{E}-\nabla \times \nabla \times \vec{E}-j \omega \mu \bar{J}_{s}\right) \cdot \delta \bar{E} d v \\
& +\int_{S} \frac{1}{j \omega} \tilde{H} \times \delta \bar{E} \cdot \bar{n} d a \\
& \equiv 0 .
\end{aligned}
$$

The boundary condition, by which the surface integral vanishes, can be met by either (1) prescribing tangential electric fields on the sirface, or, (2) forcing zero tangential magnetic fields on the surface. When one of these boundary conditions is satisfied, the electric field in the volume becomes subject to the differential equation

$$
k^{2} \bar{E}-\nabla \times \nabla \times E=j \omega \mu \bar{J}_{s} .
$$




\section{APPENDIX II}

\section{Soft Boundary}

\section{a) Modification of conductivity}

The name "soft boundary" has been given to the artificial smoothing of the conductivity across an internal boundary. The continuous conductivity change permits a continuously changing normal component of electric fields across the boundary.

An exponential transition of conductivity change is forced to occur within the strip bounded by two dotted lines shown by Figure II-1.

The composite conductivity $\sigma(x)$ between $x=x_{1}$ and $x=x_{2}$ is given by

$$
\sigma(x)=\sigma_{2}-\frac{\sigma_{2}-\sigma_{1}}{2} e^{-\alpha\left(x-x_{1}\right)}-\frac{\sigma_{2}-\sigma_{3}}{2} e^{\alpha\left(x-x_{2}\right)}
$$

where the arbitrary constant $\alpha$ is a damping factor characterizing how rapidly the transition takes place. Similarly, the composite conductivity $\sigma(z)$, Figure II-1-(b), between $z=z_{2}$ and $z=z_{3}$ is given by

$$
\sigma(z)=\sigma_{2}-\frac{\sigma_{2}-\sigma_{1}}{2} e^{-\alpha\left(z-z_{2}\right)}-\frac{\sigma_{2}-\sigma_{3}}{2} e^{\alpha\left(z-z_{3}\right)}
$$

If $\sigma_{7}=0$, the free space conductivity, we cannot use equation (2) because $\sigma(z)$ has to be zero at $z=z_{2}$. The composite conductivity $\sigma(z)$ in this case is defined by

$$
\sigma(z)=\sigma_{2}-\sigma_{2} e^{-\alpha(z-z)}-\frac{\sigma_{2}-\sigma_{3}}{z} e^{\alpha(z-z z)},
$$

in which $\sigma(z)$ becomes effectively zero at $z=z_{2}$ regardless of the coefficient of the last term provided that the exponent $\alpha\left(z-z_{3}\right)$ is a Targe negative number.

The two-dimensional composite conductivity $\sigma(x, z)$ in the region $x_{1} \leqslant x \leqslant x_{2}, z_{2} \leqslant z \leqslant z_{3}$, can be easily formulated by extending the concept 
used for $\sigma(x)$ and $\sigma(z)$. The one-dimensional composite conductivities $\sigma_{A}(x), \sigma_{B}(x)$, and $\sigma_{C}(x)$, Figure II-I- $(c)$, are first derived in places of $\sigma_{2}, \sigma_{q}$, and $\sigma_{6}$, respectively. They are

$$
\begin{aligned}
& \sigma_{A}(x)=\sigma_{2}-\frac{\sigma_{2}-\sigma_{1}}{2} e^{-\alpha\left(x-x_{1}\right)}-\frac{\sigma_{2}-\sigma_{3}}{2} e^{\alpha\left(x-x_{2}\right)}, \\
& \sigma_{B}(x)=\sigma_{9}-\frac{\sigma_{9}-\sigma_{8}}{2} e^{-\alpha\left(x-x_{1}\right)}-\frac{\sigma_{9}-\sigma_{4}}{2} e^{\alpha\left(x-x_{2}\right)}, \\
& \sigma_{C}(x)=\sigma_{6}-\frac{\sigma_{6}-\sigma_{7}}{2} e^{-\alpha\left(x-x_{1}\right)}-\frac{\sigma_{6}-\sigma_{5}}{2} e^{\alpha\left(x-x_{2}\right)}
\end{aligned}
$$

These composite conductivities are again used for the composition of the two-dimensional conductivity $\sigma(x, z)$ eventually substituting for the original $\sigma_{q}$. Hence, we obtain

$$
\sigma(x, z)=\sigma_{B}(x)-\frac{\sigma_{B}(x)-\sigma_{A}(x)}{2} e^{-\alpha\left(z-z_{2}\right)}-\frac{\sigma_{B}(x)-\sigma_{C}(x)}{2} e^{\alpha\left(z-z_{3}\right)}
$$

for $\sigma_{A}(x) \neq 0$, and

$$
\sigma(x, z)=\sigma_{B}(x)-\sigma_{B}(x) e^{-\alpha\left(z-z_{z}\right)}-\frac{\sigma_{B}(x)-\sigma_{C}(x)}{2} e^{\alpha(z-z, z)}
$$

for $\sigma_{A}(x)=0$.

The composite conductivity for a three-dimensional block can also be formulated by extending the concept one step further.

b) Variational integral with composite conductivity

From an inspection of equations (III-13), (III-14), and (III-15), we find that the only integration associated with the conductivity is

$$
\int_{S} \sigma(x, z) N \cdot N^{T} d x d z \text {. }
$$

There is one basic integration required for the evaluation of integral (9), which is 
(a)

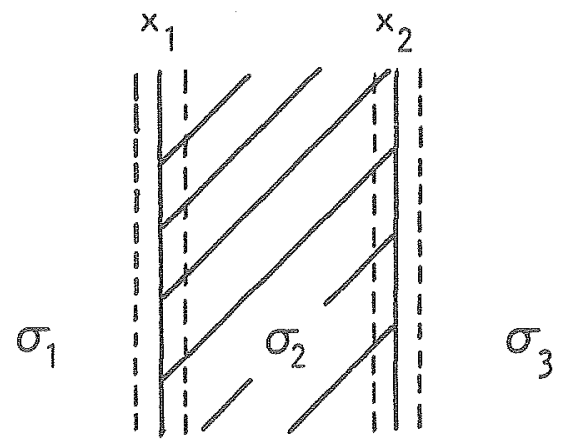

(b)

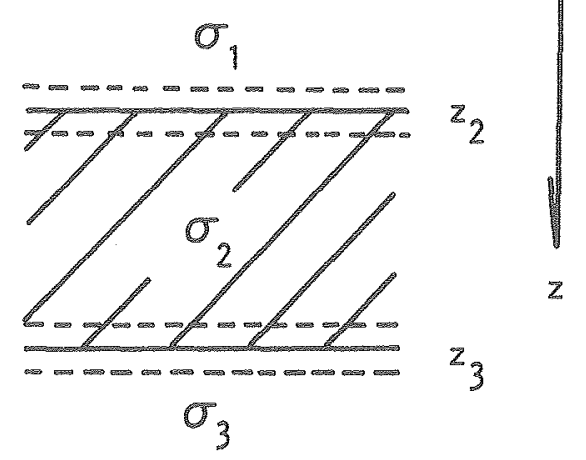

(c)

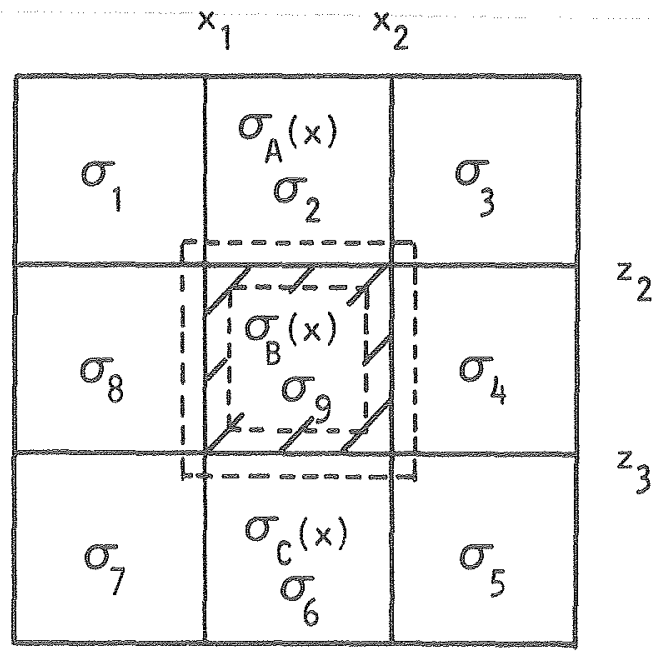

Figure 11-1. Composite conductivity 


$$
\int_{0}^{T} e^{ \pm \alpha t} t^{x} d t, \quad n=0,1,2 .
$$

Since the solution for $n=0$ is given by

$$
\pm \frac{1}{\alpha}\left(e^{ \pm \alpha T}-1\right)
$$

the solutions for $n=1,2$ may be found successively using

$$
\int_{0}^{T} e^{ \pm x t} t^{n} d t= \pm\left.\frac{1}{\alpha} e^{ \pm \alpha t} t^{n}\right|_{0} ^{T} \mp \frac{1}{\alpha} \int_{0}^{T} e^{ \pm \alpha t} t^{n-1} d t .
$$


The Harmonic Electromagnetic Field due to a Magnetic Dipole

The magnetic dipole located on or above an $\mathrm{N}$-layered half-space, Figure III-l, generates a magnetic Hertz potential $\pi^{*}$ satisfying

$$
\nabla^{2} \pi^{*}+k^{2} \pi^{*}=-\vec{m}_{s} \delta\left(x-x^{\prime}\right) \delta(y) \delta\left(z-z^{\prime}\right) 。
$$

The particular solution of equation (1) can be found by initially Fourier transforming both sides of the equation. The solution in wave number domain is given by

$$
\bar{\pi}^{p}\left(k_{x}, k_{y}, k_{z}\right)=\frac{\bar{m}_{s}}{k_{x}^{2}+k_{y}^{2}+k_{z}^{2}-k^{2}} e^{-j k_{x} x^{\prime}} e^{-j k_{z} z^{\prime}} .
$$

Inversely Fourier transforming equation (2) in $k_{z}$ and $k_{x}$ successively (Erdeiyi, 1954), we obtain

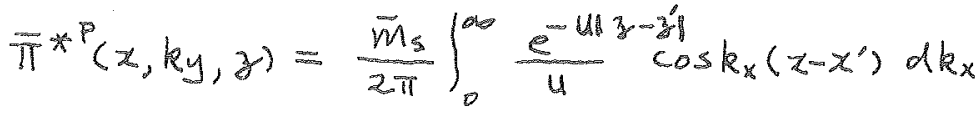

$$
\begin{aligned}
& =\frac{\bar{M}_{s}}{2 \pi} K_{0}(\gamma r) \text {, }
\end{aligned}
$$

where

$$
\begin{aligned}
& u=\left(k_{x}^{2}+k_{y}^{2}-k^{2}\right) 1 / 2 \\
& y=\left(k_{y}^{2}-k^{2}\right) 1 / 2 \\
& r=\left\{\left(x-x^{\prime}\right)^{2}+\left(z-y^{\prime}\right)^{2}\right\} 1 / 2
\end{aligned}
$$

The electromagnetic field vectors in $k_{y}$ can be found using

$$
\begin{aligned}
& E\left(k_{y}\right)=-j \omega \mu \nabla \times \pi^{*}\left(k_{y}\right), \\
& \bar{H}\left(k_{y}\right)=k^{2} \pi^{*}\left(k_{y}\right)+\nabla\left(\nabla \cdot \pi^{*}\left(k_{y}\right)\right),
\end{aligned}
$$

with $\frac{\partial}{\partial y}$ and $\frac{\partial^{2}}{\partial y^{2}}$ in (4) and (5) substituted by jky and $-k_{y}^{2} y$, respective1v. 


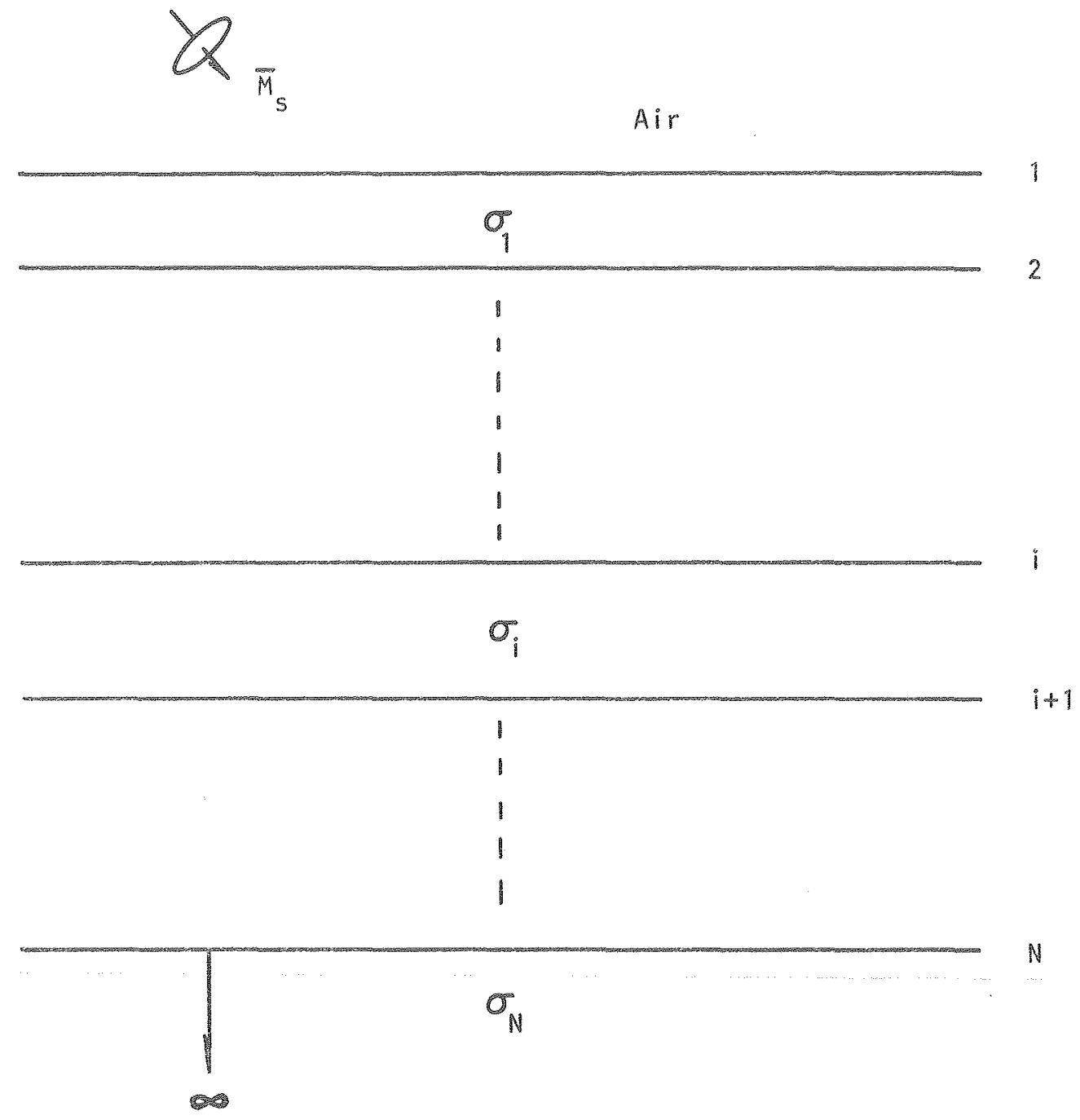

Figure 111-1. An $\mathrm{N}$-layered half space

XEL 789-10966 
In the presence of an $\mathrm{N}$-layered half-space, the vector potential $\pi^{*}(k, y)$ is written as

$$
\pi^{*}\left(k_{y}\right)=\pi^{*}\left(k_{y}\right)+\pi^{*}(k y),
$$

where $\bar{\pi}^{*}\left(k_{y}\right)$ is the secondary Hertz potential, and it satisfies the homogeneous equation

$$
\nabla^{2} \pi^{*}\left(k_{y}\right)+k^{2} \pi^{*}(k y)=0
$$

In view of the primary solution (3), $\pi^{*}\left(k_{y}\right)$ has a solution of the form

$$
\pi^{* 5}\left(k_{y}\right)=\frac{m_{3}}{2 \pi} \int_{0}^{\infty} A^{ \pm}\left(k_{x}, k_{y}\right) e^{\mp v_{i} z}\left(\begin{array}{l}
\cos k_{x}\left(x-x^{\prime}\right) \\
\sin k_{x}\left(x-x^{\prime}\right)
\end{array}\right) d k_{x}
$$

in the $i^{\text {th }}$ layer. The signs attached to $A$ indicate whether the wave is traveling down $(+)$ or up $(-)$.

a) Vertical magnetic dipole

The Hertz potential above and below the earth surface can be written as, after dropping $\left(k_{y}\right)$ and "**",

$$
\begin{aligned}
& \pi_{0}=\bar{i}_{z} \frac{m_{z}}{2 \pi} \int_{0}^{\infty}\left(\frac{e^{-u_{0} \mid z-z^{\prime}}}{u_{0}}+A_{0}^{-} e^{u_{0} z+u_{0} z^{\prime}}\right) \cos k_{x}\left(x-x^{\prime}\right) d k_{x}, z \leqslant 0, \text { (9) } \\
& \bar{\pi}_{i}=\bar{i}_{z} \frac{m_{z}}{2 \pi} \int_{0}^{\infty}\left(A_{i}^{+} e^{-u_{i} z}+A_{i}^{-} e^{u_{i} z}\right) e^{u_{0} z^{\prime}} \cos k_{x}\left(x-x^{\prime}\right) d k_{x}, z \leqslant z \leqslant z_{i+1}, \\
& \bar{\pi}_{N}=\bar{i}_{z} \frac{m_{z}}{2 \pi} \int_{0}^{\infty} A_{N}^{+} e^{-u_{N} z+u_{0} z^{\prime}} \cos k_{x}\left(x-x^{\prime}\right) d k_{x}, z_{N} \leqslant z,
\end{aligned}
$$

where the coefficients $A_{j}, j=0 \sim N$, are functions of $k_{x}$ for fixed $k_{y}$.

The electromagnetic fields can be found by equations (4) and (5). The continuities of the tangential components of electric and magnetic fields at the $i^{\text {th }}$ interface provide us the relationship between $A_{2}$ and 
$A_{i-1}$. It can be shown that for every wave number $k_{x}$, we have

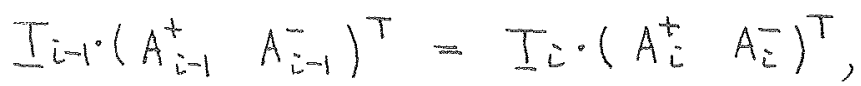

where

$$
I_{i}=\left|\begin{array}{cc}
-u_{i} & u_{i} \\
1 & 1
\end{array}\right| \cdot\left|\begin{array}{cc}
e^{-u_{i} z_{i}} & 0 \\
0 & e^{u_{i} z_{i}}
\end{array}\right|,
$$

and

$$
I_{i-1}=\left|\begin{array}{cc}
-u_{i-1} & u_{i-1} \\
1 & 1
\end{array}\right| \cdot\left|\begin{array}{cc}
e^{-u_{i-1} z_{i}} & 0 \\
0 & e^{u_{i-1} z_{i}}
\end{array}\right|
$$

Equation (12) gives a recursion formula relating the coefficients in one layer to those in the next layer by

$$
\left(\begin{array}{ll}
A_{i-1}^{+} & A_{i-1}^{-}
\end{array}\right)=Z_{i} \cdot\left(\begin{array}{ll}
A_{i}^{+} & A_{i}^{-}
\end{array}\right)
$$

where

$$
\begin{aligned}
Z_{i} & =I_{i+1}^{-1} I_{i} \\
& =\frac{1}{2 u_{i-1}}\left|\begin{array}{cc}
\left(u_{i-1}+u_{i}\right) \cdot & \left(u_{i-1}-u_{i}\right) \cdot \\
e^{\left(u_{i-1}-u_{i}\right) z_{i}} & e^{\left(u_{i-1}+u_{i}\right) z_{i}} \\
\left(u_{i-1}-u_{i}\right) . & \left(u_{i-1}+u_{i}\right) \cdot \\
e^{-\left(u_{i-1}+u_{i}\right) z_{i}} & e^{-\left(u_{i-1}-u_{i}\right) z_{i}}
\end{array}\right| .
\end{aligned}
$$

At the air-to-earth interface, the continuity conditions give similar equation to (12) as

$$
A_{0}^{-}\left(u_{0} 1\right)^{\top}=\frac{m_{3}}{2 \pi}\left(1-\frac{1}{u_{0}}\right)+I_{1} \cdot\left(A_{1}^{+} A_{1}^{-}\right)^{\top} .
$$


Since $\left(A_{T}^{+} A_{1}{ }^{-}\right)^{\top}$ can be related to $A_{N}^{+}$by recursively operating equation (13), the last equation becomes

$$
A_{0}^{-}\left(u_{0} 1\right)^{T}=\frac{m_{3}}{2 \pi}\left(1-\frac{1}{u_{0}}\right)^{\top}+I_{1} \cdot\left[\prod_{j=2}^{N} z_{j}\right] \cdot\left(A_{N}^{+} 0\right)^{\top},
$$

from which $A_{0}{ }^{-}$and $A_{N}{ }^{+}$can be computed. The coefficients $A_{j}{ }^{+}$and $A_{j}{ }^{-}$, $j=L N-1$, can be found using equation (12).

b) Horizontal magnetic dipole

The secondary Hertz potential $\pi^{*}(k y)$ in this case has to be supported by an additional zudirected potential due to the asymmetry created by the orientation of dipole moment with respect to the half space geometry.

The total potential in the regions of interest can be written in integral forms as

$$
\begin{aligned}
& \bar{\pi}_{0}=\bar{i}_{x} \frac{m_{x}}{2 \pi} \int_{0}^{\infty}\left(\frac{e^{-u_{0}|z-z|}}{u_{0}}+B_{0}^{-} e^{u_{0} z+u_{0} z}\right) \cos k_{x}\left(x-x^{\prime}\right) d k_{x} \\
& +\bar{i}_{z} \frac{m_{x}}{2 \pi} \int_{0}^{\infty} A_{0} e^{u_{0} z+u_{0} z^{\prime}} \sin k_{x}\left(x-x^{\prime}\right) d k_{x}, z \leqslant 0, \\
& \pi_{i}=\Sigma_{x} \frac{m_{x}}{2 \pi} \int_{0}^{\infty}\left(B_{i}+e^{-u_{i} z}+B_{i} e^{u_{i} z}\right) e^{u_{0} z^{\prime}} \cos k_{x}\left(x-x^{\prime}\right) d k_{x} \\
& +\Sigma_{z} \frac{m_{x}}{2 \pi} \int_{0}^{\phi_{0}}\left(A_{i}^{+} e^{-4 i z_{3}}+A_{i} e^{4 i z}\right) e^{n_{0} z^{\prime}} \sin k_{x}\left(x-x^{\prime}\right) d k_{x}, z_{i} \leq z \leq z_{i}+1 \text {, } \\
& \bar{\pi}_{N}=\bar{L}_{x} \frac{m_{x}}{2 \pi} \int_{0}^{\infty} B_{N}^{+} e^{-n_{N} z+n_{0} z^{\prime}} \cos k_{x}\left(x-x^{\prime}\right) d k_{x} \\
& +\bar{i}_{z} \frac{m_{x}}{2 \pi} \int_{0}^{\infty+\infty} A_{N}^{+} e^{-u_{N} z+u_{0} z^{\prime}} \sin k_{x}\left(x-x^{\prime}\right) d k_{x}, z_{N} \leqslant z .
\end{aligned}
$$

The selection of $\sin k_{x}\left(x-x^{\prime}\right)$ for the z-directed potential has been based on the symmetry condition of the electromagnetic field in $x$. 
Matching boundary conditions at the $i^{\text {th }}$ interface we obtain

$$
I_{i-1} \cdot\left(B_{i-1}^{+} B_{i-1}^{-} A_{i-1}^{+} A_{i-1}\right)^{\top}=\operatorname{Ii} \cdot\left(B_{i}^{+} B_{i} A_{i}^{+} A_{i}^{-}\right)^{\top},
$$

where

$$
I_{i}=\left|\begin{array}{cccc}
k_{i}^{2} & k_{i}^{2} & 0 & 0 \\
-k_{x} & -k_{x} & -u_{i} & u_{i} \\
0 & 0 & 1 & 1 \\
-u_{i} & u_{i} & 0 & 0
\end{array}\right| \cdot\left|\begin{array}{cccc}
e^{-u_{i} z_{i}} & 0 & 0 & 0 \\
0 & e^{u_{i} z_{i}} & 0 & 0 \\
0 & 0 & e^{-u_{i} z_{i}} & 0 \\
0 & 0 & 0 & e^{u_{i} z_{i}}
\end{array}\right|,
$$

and

$$
I_{i-1}=\left|\begin{array}{cccc}
k_{i-1}^{2} & k_{i-1}^{2} & 0 & 0 \\
-k_{x} & -k_{x} & -u_{i-1} & u_{i-1} \\
0 & 0 & 1 & 1 \\
-u_{i-1} & u_{i-1} & 0 & 0
\end{array}\right| \cdot\left|\begin{array}{cccc}
e^{-u_{i-1} z_{i}} & 0 & 0 & 0 \\
0 & e^{u_{i-1} z_{i}} & 0 & 0 \\
0 & 0 & e^{-u_{i-1} z_{i}} & 0 \\
0 & 0 & 0 & e^{u_{i-1} z_{i}}
\end{array}\right| .
$$

The recursion formula can be found from (19) as

$$
\left(B_{i=1}^{+} B_{i-1}^{-} A_{i-1}^{+} A_{i-1}^{-}\right)^{\top}=Z_{i} \cdot\left(B_{i}^{+} B_{i}^{-} A_{i}^{+} A_{i}\right)^{\top} \text {, }
$$

where

$$
\begin{aligned}
& Z_{i}=I_{i-1}^{-1} \cdot I_{i}
\end{aligned}
$$

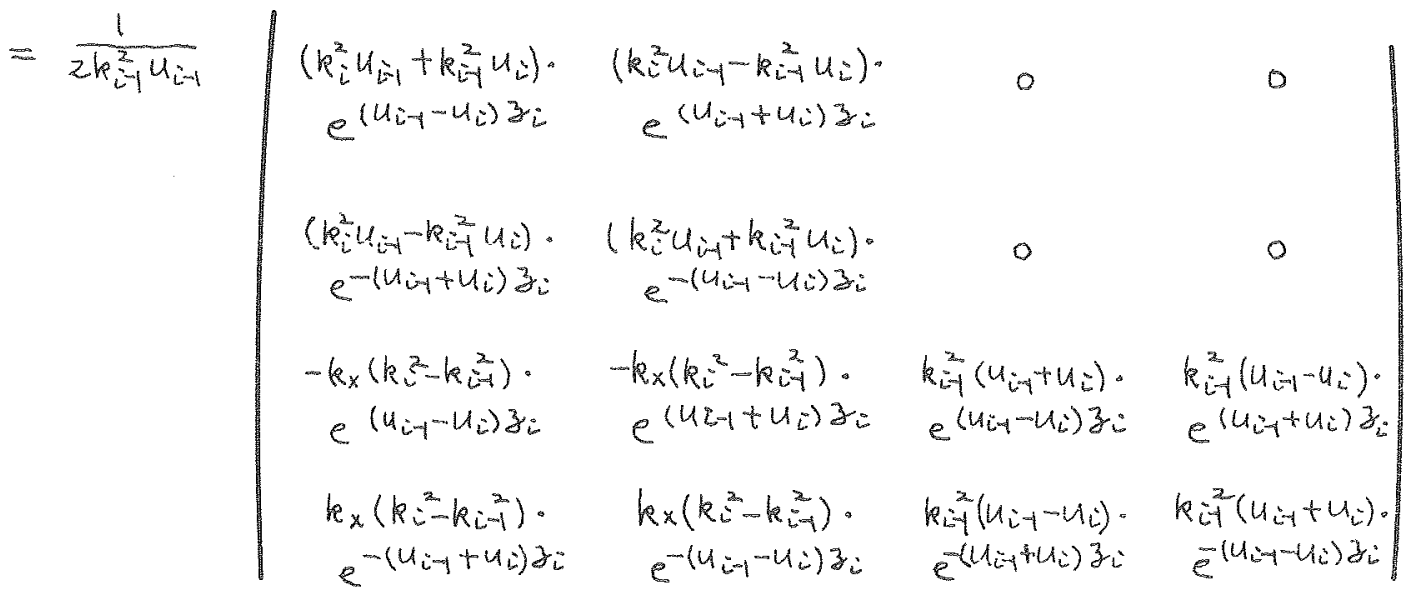

At $z=z_{1}$, the matching of boundary conditions gives a similar relationship to equation (19). After the coefficients $B_{7}^{+}, B_{7}{ }^{-}, A_{7}{ }^{+}$, and $A_{7}{ }^{-}$are related to $B_{N}^{+}$and $A_{N}{ }^{+}$by recursively substituting equation 
(20), the following set of equations are finally reached.

$$
\left|\begin{array}{cc}
k_{0}^{2} & 0 \\
-k_{x} & u_{0} \\
0 & 1 \\
u_{0} & 1
\end{array}\right| \cdot\left|\begin{array}{c}
B_{0}^{-} \\
A_{0}^{-}
\end{array}\right|=-\frac{m_{x}}{2 \pi}\left|\begin{array}{c}
\frac{k_{0}^{2}}{u_{0}} \\
-\frac{k_{x}}{u_{0}} \\
0 \\
1
\end{array}\right|+I_{1} \cdot\left[\prod_{j=2}^{N} Z_{j}\right] \cdot\left|\begin{array}{c}
B_{N}^{+} \\
0 \\
A_{N}^{+} \\
0
\end{array}\right|
$$

The four unknowns $B_{0}^{-}, A_{0}^{-}, B_{N}^{+}$, and $A_{N}{ }^{-}$can be easily found from equation (21). The unknowns in each layer can be computed by recursively operating equation (19) backward.

\section{c) Computation of the electric field}

With all the necessary coefficients found, the computation of electric field becomes trivial. Since the primary field can be derived analytically in terms of $K_{0}$ and $k_{1}$, let us find out what is the secondary electric field in the $i^{\text {th }}$ layer in the presence of a horizontal magnetic dipole.

Rewriting equation (4) for the secondary part in the $i^{\text {th }}$ layer

$$
\begin{aligned}
& E_{x_{i}}^{s}=-j \omega \mu \frac{\partial}{\partial y} \pi_{z i}^{s}, \\
& E_{y i}^{s}=-j \omega \mu\left(\frac{\partial}{\partial z} \pi_{x_{i}}^{s}-\frac{\partial}{\partial x} \pi_{z i}^{s}\right), \\
& E_{z i}^{s}=j \omega \mu \frac{\partial}{\partial y} \pi_{x_{i}}^{s},
\end{aligned}
$$

where $\pi_{x i}^{s}$ is the total $x$-component Hertz potential subtracted by the primary potential given by equation (3). The $z$-component total potential itself is the secondary potential because there is no primary potential in that direction. Hence, from equation (17) 


$$
\begin{aligned}
& \pi_{x i}^{s}=\frac{m_{x}}{2 \pi} \int_{0}^{n_{0}}\left(B_{i}^{t} e^{-u_{i} z}+B_{i} e^{u_{i} z}-\frac{1}{u_{0}} e^{-u_{0} z}\right) e^{u_{0} z^{\prime}} \cos k_{x}\left(x-x^{\prime}\right) d k_{x}, \\
& \pi_{z_{i}}^{s}=\frac{m_{x}}{2 \pi} \int_{0}^{\infty_{0}}\left(A_{i}^{\frac{1}{t}} e^{-u_{i} z}+A_{i} e^{u_{i} z}\right) e^{u_{0} z^{\prime}} \sin k_{x}\left(x-x^{\prime}\right) d k_{x} .
\end{aligned}
$$

Substituting (25) and (26) into (22), (23), and (24), we obtain

$$
\begin{aligned}
& \left.E_{x_{i}}^{s}=\omega \mu k_{y} \frac{m x}{2 \pi} \int_{0}^{p_{0}}\left(A_{i}^{t} e^{-u_{i} z}+A_{i} e^{u_{i} z}\right) e^{u_{0} z^{\prime}} \sin k_{x}\left(x_{-}-x^{\prime}\right) d k_{x}\right) \\
& \left.E_{y i}^{s}=-j \omega \mu \frac{m_{x}}{2 \pi}\right\}_{0}^{m_{0}}\left\{u_{i}\left(-B_{i}^{+} e^{-u_{i} z}+B_{i} e^{u_{i} z}\right)+e^{-u_{0} z}\right. \\
& \left.-k_{x}\left(A_{i}^{t} e^{-u_{i} z}+A_{i}^{-} e^{u_{i} z}\right)\right\} e^{u_{0} z^{\prime}} \cos k_{x}\left(x-x^{\prime}\right) d k_{x}, \\
& E_{z i}^{s}=-\omega \mu k_{y} \frac{m_{k}}{2 \pi} \int_{0}^{x_{i}}\left(B_{i}^{t} e^{-u_{i} z}+B_{i} e^{+u_{i} z}-\frac{1}{u_{0}} e^{-n_{0} z}\right) . \\
& e^{u \cdot z^{\prime}} \cos k_{x}\left(x-x^{\prime}\right) d k_{x}
\end{aligned}
$$

The integration in $k_{x}$ has been carried out numerically up to a certain number of periods until the desired convergence criterion would be met. The subtractive primary term $e^{-u_{0}} / u_{0}$ in $(25)$, which otherwise could be evaluated analytically, actually accelerates the rate of convergence of numerical integration. 


\section{REFERENCES:}

Abramowitz, M., and Stegun, I. A., 1964, Handbook of Mathematical

Functions: N.B.S., U.S. Government Printing office.

Coggon, J. H., 1971, Electromagnetic and electrical Modelling by the Finite Element Method: Geophysics, Vol. 36, No. 1, p. 132.

Dey, A.s and Ward, S. H., 1970, Inductive Sounding of a Layered Earth with a Horizontal Magnetic Dipole: Geophysics, Vol. 35 , No. 4 , p. 660 .

Erdelyi, A., et a1, 1954, Tables of Integral Transforms: New York, McGraw-Hill.

Frischknecht, F.C., 1967, Fields about an Oscillating Magnetic Dipole over a Two-Layer Earth: Colo. School of Mines Quart., Vol. 62, No. 1.

Grant, F. S., and West, G. F., 1965, Interpretation Theory in Applied Geophysics: New York, McGraw-Hill.

Gröbner, W., and Hofreiter, N., 1958, Integraltafel, Teil II, Bestimmte Integrale: Wien, Springer-Verlag.

Guar, V. K., Verma, 0.P., and Gupta, C. P., 1972, Enhancement of Electromagnetic Anomalies by a Conducting Overburden: Geophys. Prosp., Vol. 20, p. 580.

Harrington, R. F., 1961, Time-Harmonic Electromagnetic Fields: New York, McGraw-Hill.

Hildebrand, F. B., 1965, Methods of Applied Mathematics: New Jersey, Prentice-Hall.

Hohmann, G. W. 1971, Electromagnetic Scattering by Conductors in the Earth near a Line Source of Current: Geophysics, Vol. 36, p. 101.

Hohmann, G. W. 1975, Three Dimensional Induced Polarization and Electromagnetic Modeling: Geophysics, Vol, 40, p. 309.

Jackson, J. D., 1967, Classical Electrodynamics: New York, John Wiley \& Sons.

Martin, R. S., and Wilkinson, J. H., 1965, Symmetric Decomposition of Positive Definitive Band Matrices: Numer. Math. Vol. 7, p. 355.

Meyer, W. H. 1977, Computer Model1ing of Electromagnetic Prospecting Methods: Berkeley, Ph.D. Thesis, University of California. 
Parry, J.R., and Ward, S. H., 1971, Electromagnetic Scattering from Cylinders of Arbitrary Cross-Section in a Conductive Half-Space: Geophysics, Vol. 36, p. 67.

Pridmore, D. F., 1978, Three Dimensional Modelling of Electric and Electromagnetic Data Using the Finite Element Method: Ph.D. Thesis, The University of Utah.

Reid, J. K., 1972. Two Fortran Subroutines for Direct Solution of Linear Equations Whose Matrix is Sparse. Symmetric and PositiveDefinite: U.R.A.E.A. Research Group Report AERE-R7119.

Ryu, J., Morrison, H. F., and Ward, S. H., 1970, Electromagnetic Fields about a Loop Source of Current: Geophysics, Vol. 35, p. 862.

Ryu. J., 1971, Low Frequency Electromagnetic Scattering: Berkeley, Ph.D. Thesis, University of California.

Sarma, D. G., and Maru, J. M. 1971. A Study of Some Effects of a Conducting Host Rock with a New Modeling Apparatus: Geophysics, Vol. 36, p. 166.

Sommerfeld, A., 1967, Partial Differential Equations in Physics: New York, Academic Press.

Stoyer, C. H. 1974, Numerical Solutions of the Response of a TwoDimensional Earth to an Oscillating Magnetic Dipole Source with Application to a Groundwater Field Study: Ph.D. Thesis. The Pennsylvania State University.

Stratton, J. A., 1941, Electromagnetic Theory: New York, McGraw-Hill.

Swift, C. M. 1967, A Magnetotelluric Investigation of an Electrical Conductivity Anomaly in the South-Western United States: Ph.D. Thesis. Massachusetts Institute of Technology.

Wait, J. R. 1962, Electromagnetic Waves in Stratified Pedia: New York, Macmilian.

Ward, S. H., 1967, Electromagnetic Theory for Geophysical Application: Tulsa, Soc. of Expl. Geoph. Mining Volume II.

Weidelt, P., 1975, Electromagnetic Induction in Three Dimensional Structures: J. Geophysics, Vol. 41, p.85.

Zienkiewicz, 0. C., 1971, The Finite Element Method in Engineering Science: London, McGraw-Hill. 
This report was done wilh suppont hom the Deputinent of Linergy. Any oonclusions or aphions expressed in this report represter soldy Those of the atuthor(s) and not necersart) whose of the hegrents of tho

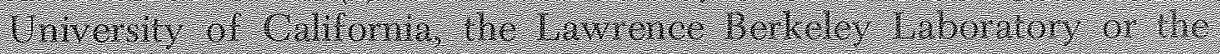
Department at Tinergy: 


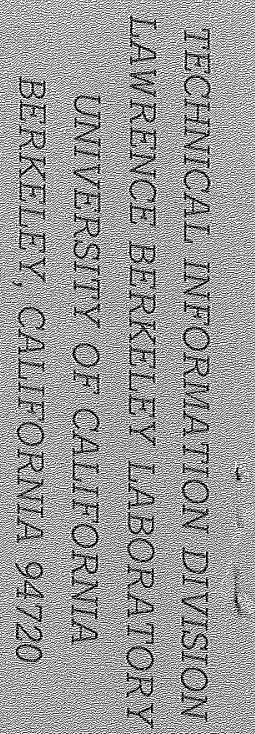

